\title{
Copper-Mediated Remote C-H Bond Chalcogenation of Quinolines on C5 Position
}

Longzhi Zhu, ${ }^{\dagger}$ Renhua Qiu, ${ }^{*}{ }^{\dagger}$ Xin Cao,${ }^{\dagger}$ Song Xiao, ${ }^{\dagger}$ Xinhua Xu, ${ }^{\dagger}$ Chak-Tong Au, ${ }^{\dagger}$, and Shuang-Feng Yin ${ }^{*}, \dagger$

\author{
'State Key Laboratory of Chemo/Biosensing and Chemometrics, College of Chemistry and Chemical \\ Engineering, Hunan University, Changsha, 410082, P. R. China

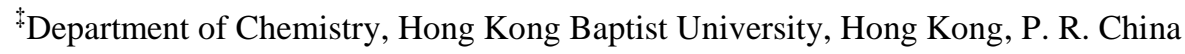 \\ renhuaqiu@hnu.edu.cn (Dr. Qiu); sf_yin@hnu.edu.cn (Prof. Yin)
}

\section{Table of Contents}

1. General 


\section{General}

\section{Information}

All reactions were carried out under $\mathrm{O}_{2}$ atmosphere using standard Schlenk techniques unless stated otherwise. The glassware was dried in an oven $\left(110^{\circ} \mathrm{C}\right)$ and heated under reduced pressure before use. The thin layer chromatography (TLC) analyses and column chromatography were performed using Anhui Liangchen silica gel (200-300) with distilled solvents. NMR spectra were recorded on a Bruker Avance 400 spectrometer operating at 400 $\mathrm{MHz}\left({ }^{1} \mathrm{H}\right.$ NMR $)$ and $101 \mathrm{MHz}\left({ }^{13} \mathrm{C} \mathrm{NMR}\right)$ in $\mathrm{CDCl}_{3}$. All ${ }^{1} \mathrm{H}$ and ${ }^{13} \mathrm{C}$ NMR chemical shifts were reported in ppm relative to internal references of $\left(\mathrm{CH}_{3}\right)_{4} \mathrm{Si}$ at $7.26 \mathrm{ppm}$ and carbon resonance in chloroform- $d_{1}$ at $77.00 \mathrm{ppm}$, respectively. The following abbreviations are used to describe peak patterns where appropriate: singlet $(\mathrm{s})$, doublet $(\mathrm{d})$, triplet $(\mathrm{t})$, multiplet $(\mathrm{m})$, broad resonances (br).

\section{Chemicals}

Unless noted otherwise, the materials obtained from commercial suppliers were used without further purification. All solvents were redistilled.

\section{Typical Experimental Procedure for the Preparation of Starting materials Aminoquinolines Derivatives (1)}

The acid chloride $(1.1 \mathrm{mmol})$ was added dropwise to a solution of 8 -aminoquinoline $(1.44 \mathrm{~g}$, $10.0 \mathrm{mmol})$ and $\mathrm{Et}_{3} \mathrm{~N}(1.7 \mathrm{~mL}, 12 \mathrm{mmol})$ in $\mathrm{CH}_{2} \mathrm{Cl}_{2}(15 \mathrm{~mL})$ at $0{ }^{\circ} \mathrm{C}$. The mixture was stirred overnight at room temperature. Then the mixture was diluted with $\mathrm{CH}_{2} \mathrm{Cl}_{2}(10 \mathrm{~mL})$, and washed successively with water, saturated aqueous $\mathrm{NaHCO}_{3}$, and brine. The organic layer was dried over $\mathrm{MgSO}_{4}$ and concentrated under reduced pressure. The residue was purified by flash column chromatography on silica gel and eluted with EtOAc/Hexane $(1: 10, \mathrm{v} / \mathrm{v})$ to afford the corresponding 8-aminoquinolinyl amides 1. Starting materials were prepared according to literature procedures. ${ }^{1}$

\section{General Procedure for the Thiolation/ Selenylation of Quinolines}

To a $10 \mathrm{~mL}$ screw capped vial equipped with a magnetic stirring bar was added aminoquinolines derivatives (1) $(0.3 \mathrm{mmol})$, disulfide derivatives/ diaryl diselenides (2) (0.36 $\mathrm{mmol}), \mathrm{CuBr}_{2}(0.45 \mathrm{mmol})$, and DMF $(1 \mathrm{~mL})$ under $\mathrm{O}_{2}$ atmosphere. The reaction mixture was placed in a pre-heated oil bath at $160{ }^{\circ} \mathrm{C}$ and vigorously stirred for $24 \mathrm{~h}$. Afterward it was cooled to ambient temperature, filtered through a plug of celite and then washed with ethyl acetate $(3 \times 5 \mathrm{~mL})$. The solvents were removed under reduced pressure and the crude reaction mixture was purified by chromatography on silica gel ( $n$-hexane/EtOAc) as an eluent to obtain the desired product. In some cases, the products were purified by the PTLC method. 


\section{Optimization of Additives}

Table S1 Optimization of the amount of $\mathrm{CuBr}_{2}{ }^{a}$

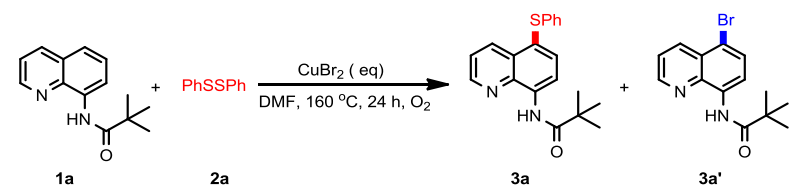

\begin{tabular}{lllll}
\hline Entry & $\mathrm{CuBr}_{2}(\mathrm{eq})$ & Sol. $(1 \mathrm{~mL})$ & Temp. $\left({ }^{\circ} \mathrm{C}\right)$ & Yield $^{b}(\mathbf{3 a} / \mathbf{3 a})$ \\
\hline 1 & 0 & DMF & 160 & 0 \\
2 & 0.1 & $\mathrm{DMF}$ & 160 & 10 \\
3 & 0.2 & $\mathrm{DMF}$ & 160 & 18 \\
4 & 0.5 & $\mathrm{DMF}$ & 160 & 38 \\
5 & 1 & $\mathrm{DMF}$ & 160 & $78 / 3$ \\
6 & 1.5 & $\mathrm{DMF}$ & 160 & $89(86) / 5$ \\
7 & 2 & $\mathrm{DMF}$ & 160 & $90 / 8$ \\
\hline
\end{tabular}

${ }^{a}$ Reaction conditions: ${ }^{a}$ 1a $(0.3 \mathrm{mmol}), \mathbf{2 a}(0.36 \mathrm{mmol}), \mathrm{CuBr}_{2}$, solvent $(1.0 \mathrm{~mL}), \mathrm{O}_{2}$ atmosphere; ${ }^{b} \mathrm{GC}$ yield, using $n$-tridecane as internal standard; the number in parentheses is isolated yield

Table S2 Optimization of the acid additives ${ }^{a}$

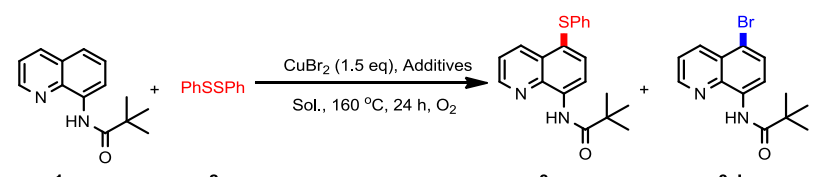

3a

3a'

\begin{tabular}{lllll}
\hline Entry & Additives $(2 \mathrm{eq})$ & Sol. $(1 \mathrm{~mL})$ & Temp. $\left({ }^{\circ} \mathrm{C}\right)$ & Yield $^{b}\left(\mathbf{3 a} / \mathbf{3 a} \mathbf{a}^{\prime}\right)$ \\
\hline 1 & PhCOOH & DMF & 160 & $72 \%$ \\
2 & MesCOOH & DMF & 160 & $65 \%$ \\
3 & $\mathrm{CH}_{3} \mathrm{COOH}$ & DMF & 160 & $73 \%$ \\
4 & PivOH & DMF & 160 & $71 \%$ \\
5 & Phenylphosphonic acid & DMF & 160 & $25 \%$ \\
6 & $1-$ Adamantanecarboxylic acid & DMF & 160 & $62 \%$ \\
7 & -- & DMF & 160 & $89(86) / 5$ \\
\hline
\end{tabular}

${ }^{a}$ Reaction conditions: ${ }^{a}$ 1a $(0.3 \mathrm{mmol}), \mathbf{2 a}(0.36 \mathrm{mmol}), \mathrm{CuBr}_{2}(1.5 \mathrm{eq}), \mathrm{DMF}(1.0 \mathrm{~mL}), \mathrm{O}_{2}$ atmosphere; ${ }^{b}$ GC yield, using $n$-tridecane as internal standard. 
Table S3 Optimization of the basic additives ${ }^{a}$

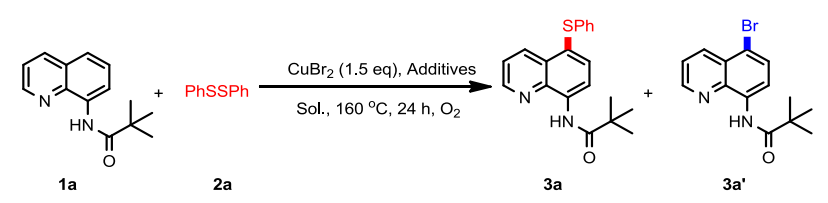

\begin{tabular}{|c|c|c|c|c|}
\hline Entry & Additives ( eq) & Sol.(1 mL) & Temp.$\left({ }^{\circ} \mathrm{C}\right)$ & Yield $^{b}\left(\mathbf{3 a} / \mathbf{3 a} \mathbf{a}^{\prime}\right)$ \\
\hline 1 & $\mathrm{Na}_{2} \mathrm{CO}_{3}$ & DMF & 160 & $57 \%$ \\
\hline 2 & $\mathrm{~K}_{2} \mathrm{CO}_{3}$ & DMF & 160 & $20 \%$ \\
\hline 3 & $\mathrm{KHCO}_{3}$ & $\mathrm{DMF}$ & 160 & $21 \%$ \\
\hline 4 & $\mathrm{Cs}_{2} \mathrm{CO}_{3}$ & DMF & 160 & $19 \%$ \\
\hline 5 & $\mathrm{~K}_{3} \mathrm{PO}_{4}$ & DMF & 160 & $13 \%$ \\
\hline 6 & $\mathrm{KH}_{2} \mathrm{PO}_{4}$ & DMF & 160 & $21 \%$ \\
\hline 7 & $\mathrm{Et}_{3} \mathrm{~N}$ & $\mathrm{DMF}$ & 160 & $26 \%$ \\
\hline 8 & KOAc & DMF & 160 & $29 \%$ \\
\hline 9 & $\mathrm{KBF}_{4}$ & DMF & 160 & $62 \%$ \\
\hline 10 & -- & DMF & 160 & $89(86) / 5$ \\
\hline
\end{tabular}

${ }^{a}$ Reaction conditions: ${ }^{a}$ 1a $(0.3 \mathrm{mmol}), 2 \mathrm{a}(0.36 \mathrm{mmol}), \mathrm{CuBr}_{2}(1.5 \mathrm{eq}), \mathrm{DMF}(1.0 \mathrm{~mL}), \mathrm{O}_{2}$ atmosphere; ${ }^{b}$ GC yield, using $n$-tridecane as internal standard.

\section{Structures of Starting Materials}

$N$-(quinolin-8-yl)pivalamide (1a)<smiles>CC(C)(C)C(=O)Nc1cccc2cccnc12</smiles>

The amide was obtained as yellow oil. ${ }^{1} \mathrm{H}$ NMR $\left(400 \mathrm{MHz}, \mathrm{CDCl}_{3}\right): \delta$ $10.27(\mathrm{~s}, 1 \mathrm{H}), 8.81-8.77(\mathrm{~m}, 2 \mathrm{H}), 8.08(\mathrm{~d}, J=8.2 \mathrm{~Hz}, 1 \mathrm{H}), 7.53-7.36$ $(\mathrm{m}, 3 \mathrm{H}), 1.42(\mathrm{~s}, 9 \mathrm{H}) .{ }^{13} \mathrm{C} \mathrm{NMR}\left(101 \mathrm{MHz}, \mathrm{CDCl}_{3}\right): \delta=177.1,148.1$, $138.6,136.1,134.5,127.8,127.2,121.4,121.1,116.0,40.2,27.6 . \mathrm{MS}(\mathrm{EI})$ : $\mathrm{m} / \mathrm{z}=228.1$.

$N$-(quinolin-5-yl)pivalamide (1aa)<smiles>CC(C)(C)C(=O)Nc1cccc2ncccc12</smiles>

The amide was obtained as yellow solid, mp: $121-122{ }^{\circ} \mathrm{C} .{ }^{1} \mathrm{H}$ NMR (400 $\left.\mathrm{MHz} \mathrm{CDCl}_{3}\right) \delta 8.85(\mathrm{~d}, J=4.0 \mathrm{~Hz}, 1 \mathrm{H}), 8.00(\mathrm{~d}, J=8.5 \mathrm{~Hz}, 1 \mathrm{H}), 7.92$ (d, $J=8.1 \mathrm{~Hz}, 1 \mathrm{H}), 7.83(\mathrm{~s}, 1 \mathrm{H}), 7.68-7.56(\mathrm{~m}, 2 \mathrm{H}), 7.33(\mathrm{dd}, J=8.5,4.1 \mathrm{~Hz}$ $1 \mathrm{H}), 1.35$ (s, 9H). ${ }^{13} \mathrm{C} \mathrm{NMR}\left(101 \mathrm{MHz}, \mathrm{CDCl}_{3}\right): \delta 177.5,150.2,148.5$, 
$N$-(quinolin-8-yl)acetamide (1b)<smiles>CC(=O)Nc1cccc2cccnc12</smiles>

The amide was obtained as yellow solid, mp: 94-96 ${ }^{\circ} \mathrm{C} .{ }^{1} \mathrm{H}$ NMR (400 MHz, $\left.\mathrm{CDCl}_{3}\right): \delta 9.78(\mathrm{~s}, 1 \mathrm{H}), 8.82-8.72(\mathrm{~m}, 2 \mathrm{H}), 8.14(\mathrm{~d}, J=8.3 \mathrm{~Hz}, 1 \mathrm{H}), 7.55-$ $7.40(\mathrm{~m}, 3 \mathrm{H}), 2.34(\mathrm{~s}, 3 \mathrm{H}) .{ }^{13} \mathrm{C} \mathrm{NMR}\left(101 \mathrm{MHz}, \mathrm{CDCl}_{3}\right): \delta=168.8,148.1$, $138.2,136.3,134.5,127.9,127.4,121.5,121.4,116.4,25.1 . \mathrm{MS}(\mathrm{EI}): \mathrm{m} / \mathrm{z}=$ 186.1

2,2-dimethyl- $N$-(quinolin-8-yl)butanamide (1c)<smiles>CCC(C)(C)C(=O)Nc1cccc2cccnc12</smiles>

The amide was obtained as yellow oil. ${ }^{1} \mathrm{H}$ NMR $\left(400 \mathrm{MHz}, \mathrm{CDCl}_{3}\right) \delta$ $10.24(\mathrm{~s}, 1 \mathrm{H}), 8.80(\mathrm{t}, J=6.8 \mathrm{~Hz}, 2 \mathrm{H}), 8.18-8.04(\mathrm{~m}, 1 \mathrm{H}), 7.51(\mathrm{t}, J=$ $7.9 \mathrm{~Hz}, 1 \mathrm{H}), 7.45(\mathrm{~d}, J=8.0 \mathrm{~Hz}, 1 \mathrm{H}), 7.41(\mathrm{dd}, J=8.2,4.2 \mathrm{~Hz}, 1 \mathrm{H})$, $1.76(\mathrm{q}, J=7.5 \mathrm{~Hz}, 2 \mathrm{H}), 1.39(\mathrm{~s}, 6 \mathrm{H}), 0.95(\mathrm{t}, J=7.5 \mathrm{~Hz}, 3 \mathrm{H}) .{ }^{13} \mathrm{C}$ NMR

$\left(101 \mathrm{MHz} \mathrm{CDCl}_{3}\right): \delta 176.6,148.1,138.7,136.2,134.6,127.8,127.3$, $121.4,121.1,116.1,44.0,34.0,25.0,9.2$. MS (EI): $\mathrm{m} / \mathrm{z}=242.2$.

2-ethyl- $N$-(quinolin-8-yl)butanamide (1d)<smiles>CCC(CC)C(=O)Nc1cccc2cccnc12</smiles>

The amide was obtained as yellow oil. ${ }^{1} \mathrm{H}$ NMR $\left(400 \mathrm{MHz}, \mathrm{CDCl}_{3}\right): \delta$ $9.84(\mathrm{~s}, 1 \mathrm{H}), 8.83$ (d, $J=7.4 \mathrm{~Hz}, 1 \mathrm{H}), 8.77$ (d, $J=3.9 \mathrm{~Hz}, 1 \mathrm{H}), 8.07$ (d, $J=8.2 \mathrm{~Hz}, 1 \mathrm{H}), 7.50-7.35(\mathrm{~m}, 3 \mathrm{H}), 2.34-2.27(\mathrm{~m}, 1 \mathrm{H}), 1.86-1.72$ $(\mathrm{m}, 2 \mathrm{H}), 1.68-1.56(\mathrm{~m}, 3 \mathrm{H}), 0.98(\mathrm{t}, J=7.4 \mathrm{~Hz}, 6 \mathrm{H}) .{ }^{13} \mathrm{C}$ NMR (101 $\mathrm{MHz}, \mathrm{CDCl}_{3}$ ): $\delta=174.7,148.0,138.2,136.2,134.2,127.7,127.2,121.4$, $121.3,116.4,52.4,25.7,24.7,11.9,11.6$. MS (EI): $\mathrm{m} / \mathrm{z}=242.2$.

$N$-(quinolin-8-yl)cyclopentanecarboxamide (1e)<smiles>O=C(Nc1cccc2cccnc12)C1CCCC1</smiles>

The amide was obtained as yellow solid, mp: 70-71 ${ }^{\circ} \mathrm{C} .{ }^{1} \mathrm{H}$ NMR (400 $\mathrm{MHz}_{\mathrm{CDCl}}$ ): $\delta 9.84(\mathrm{~s}, 1 \mathrm{H}), 8.85-8.69(\mathrm{~m}, 2 \mathrm{H}), 8.16-8.01(\mathrm{~m}, 1 \mathrm{H})$, $7.51-7.34(\mathrm{~m}, 3 \mathrm{H}), 2.97-2.82(\mathrm{~m}, 1 \mathrm{H}), 2.14-1.91(\mathrm{~m}, 4 \mathrm{H}), 1.84-$ $1.75(\mathrm{~m}, 2 \mathrm{H}), 1.71-1.58(\mathrm{~m}, 2 \mathrm{H}) .{ }^{13} \mathrm{C} \mathrm{NMR}\left(101 \mathrm{MHz}, \mathrm{CDCl}_{3}\right): \delta 174.7$, $147.7,138.0,135.9,134.3,127.5,127.0,121.1,120.9,115.9,47.00,30.2$, 25.6. MS (EI): $\mathrm{m} / \mathrm{z}=240.1$.

1-methyl- $N$-(quinolin-8-yl)cyclohexanecarboxamide (1f)<smiles>CC1(C(=O)Nc2cccc3cccnc23)CCCCC1</smiles>

The amide was obtained as yellow oil. ${ }^{1} \mathrm{H}$ NMR (400 $\left.\mathrm{MHz}, \mathrm{CDCl}_{3}\right): \delta$ $10.28(\mathrm{~s}, 1 \mathrm{H}), 8.80(\mathrm{dd}, J=18.2,5.2 \mathrm{~Hz}, 2 \mathrm{H}), 8.08(\mathrm{dd}, J=8.2,1.3 \mathrm{~Hz}$, $1 \mathrm{H}), 7.52-7.35(\mathrm{~m}, 3 \mathrm{H}), 2.27-2.11(\mathrm{~m}, 2 \mathrm{H}), 1.69-1.45(\mathrm{~m}, 8 \mathrm{H})$, 
1.35 (s, 3H). ${ }^{13} \mathrm{C}$ NMR $\left(101 \mathrm{MHz}, \mathrm{CDCl}_{3}\right): \delta 176.4,148.1,138.6,136.1,134.6,127.8,127.3$, 121.4, 121.0, 116.0, 44.2, 35.6, 26.5, 25.7, 22.9. MS (EI): $\mathrm{m} / \mathrm{z}=268.2$.

$N$-(quinolin-8-yl)octanamide (1g)

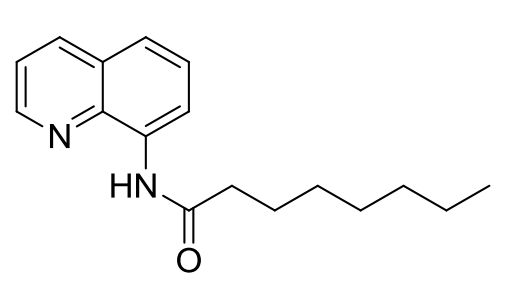

The amide was obtained as yellow oil. ${ }^{2}{ }^{1} \mathrm{H}$ NMR $(400 \mathrm{MHz}$, $\left.\mathrm{CDCl}_{3}\right): \delta 9.77(\mathrm{~s}, 1 \mathrm{H}), 8.82-8.67(\mathrm{~m}, 2 \mathrm{H}), 8.05(\mathrm{~d}, J=8.2$ $\mathrm{Hz}, 1 \mathrm{H}), 7.50-7.32(\mathrm{~m}, 3 \mathrm{H}), 2.50(\mathrm{t}, J=7.6 \mathrm{~Hz}, 2 \mathrm{H}), 1.86$ $-1.69(\mathrm{~m}, 2 \mathrm{H}), 1.45-1.19(\mathrm{~m}, 8 \mathrm{H}), 0.85(\mathrm{t}, J=6.7 \mathrm{~Hz}, 3 \mathrm{H})$. ${ }^{13} \mathrm{C}$ NMR $\left(101 \mathrm{MHz}, \mathrm{CDCl}_{3}\right): \delta 171.7,147.9,138.1,136.1$, $134.4,127.7,127.2,121.3,121.1,116.2,38.0,31.5,29.1$, 28.9, 25.5, 22.4, 13.9. MS (EI): $\mathrm{m} / \mathrm{z}=270.2$.

11-bromo- $N$-(quinolin-8-yl)undecanamide (1h)<smiles>O=C(CCCCCCCCCCBr)Nc1cccc2cccnc12</smiles>

The amide was obtained as yellow solid, mp: 67-68 ${ }^{\circ} \mathrm{C} .{ }^{3}{ }^{1} \mathrm{H}$ NMR $\left(400 \mathrm{MHz}, \mathrm{CDCl}_{3}\right): \delta 9.77(\mathrm{~s}$, $1 \mathrm{H}), 8.76(\mathrm{t}, J=6.0 \mathrm{~Hz}, 2 \mathrm{H}), 8.09(\mathrm{~d}, J=8.2 \mathrm{~Hz}, 1 \mathrm{H}), 7.57-7.33(\mathrm{~m}, 3 \mathrm{H}), 3.35(\mathrm{t}, J=6.8 \mathrm{~Hz}$, $2 \mathrm{H}), 2.52(\mathrm{t}, J=7.5 \mathrm{~Hz}, 2 \mathrm{H}), 1.85-1.71(\mathrm{~m}, 4 \mathrm{H}), 1.46-1.21(\mathrm{~m}, 12 \mathrm{H}) .{ }^{13} \mathrm{C}$ NMR $(101 \mathrm{MHz}$, $\left.\mathrm{CDCl}_{3}\right): \delta 171.6,147.9,138.1,136.1,134.4,127.7,127.2,121.4,121.1,116.2,38.0,33.9,32.6$, 29.17, 29.16, 29.15, 29.1, 28.5, 28.0, 25.5. MS (EI): $\mathrm{m} / \mathrm{z}=390.1$.

\section{Analytical Data for the Products}

$N$-(5-(phenylthio)quinolin-8-yl)pivalamide (3a)<smiles>CC(C)(C)C(=O)Nc1ccc(Sc2ccccc2)c2cccnc12</smiles>

The amide was obtained as yellow solid, $86.7 \mathrm{mg}, 86 \%$ yield. Mp: $152-153{ }^{\circ} \mathrm{C}$. ${ }^{1} \mathrm{H}$ NMR $\left(400 \mathrm{MHz}, \mathrm{CDCl}_{3}\right): \delta 10.39(\mathrm{~s}, 1 \mathrm{H}), 8.85-8.79(\mathrm{~m}, 2 \mathrm{H}), 8.67(\mathrm{~d}, J=$ $8.4 \mathrm{~Hz}, 1 \mathrm{H}), 7.89$ (d, $J=7.3 \mathrm{~Hz}, 1 \mathrm{H}), 7.46(\mathrm{dd}, J=8.5,4.2 \mathrm{~Hz}, 1 \mathrm{H}), 7.17$ (t, $J$ $=7.4 \mathrm{~Hz}, 3 \mathrm{H}), 7.11-7.02(\mathrm{~m}, 3 \mathrm{H}), 1.45(\mathrm{~s}, 9 \mathrm{H}) .{ }^{13} \mathrm{C}$ NMR $\left(101 \mathrm{MHz}, \mathrm{CDCl}_{3}\right)$ : $\delta 177.4,148.4,139.4,137.9,136.3,136.2,134.9,129.3,129.0,127.2,125.6$, 122.3, 122.3, 116.1, 40.4, 27.7. HRMS m/z (EI) calcd for $\left[\mathrm{C}_{20} \mathrm{H}_{20} \mathrm{~N}_{2} \mathrm{OS}\right]$ :

336.1296, found: 336.1299 .

$N$-(5-bromoquinolin-8-yl)pivalamide (3a')

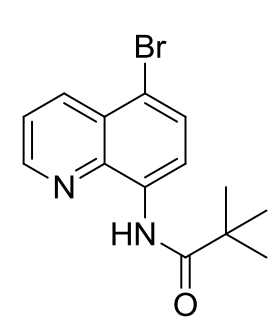

The amide was obtained as yellow solid. Mp: $114-115{ }^{\circ} \mathrm{C} .{ }^{1} \mathrm{H}$ NMR $(400$ $\left.\mathrm{MHz}, \mathrm{CDCl}_{3}\right) \delta 10.23(\mathrm{~s}, 1 \mathrm{H}), 8.83(\mathrm{dd}, J=4.1,1.2 \mathrm{~Hz}, 1 \mathrm{H}), 8.69(\mathrm{~d}, J=8.4$ 
$\mathrm{Hz}, 1 \mathrm{H}), 8.53(\mathrm{dd}, J=8.5,1.2 \mathrm{~Hz}, 1 \mathrm{H}), 7.79(\mathrm{~d}, J=8.4 \mathrm{~Hz}, 1 \mathrm{H}), 7.56(\mathrm{dd}, J=8.5,4.2 \mathrm{~Hz}, 1 \mathrm{H})$, 1.42 (s, 9H). ${ }^{13} \mathrm{C}$ NMR $\left(101 \mathrm{MHz}, \mathrm{CDCl}_{3}\right): \delta 177.3,148.5,139.3,136.1,134.5,131.0,127.2$, 122.5, 117.0, 113.9, 40.4, 27.6. HRMS m/z (EI) calcd for $\left[\mathrm{C}_{14} \mathrm{H}_{15} \mathrm{BrN}_{2} \mathrm{O}\right]: 306.0368$, found: 306.0372 .

$N$-(5-(p-tolylthio)quinolin-8-yl)pivalamide (3b)<smiles>Cc1ccc(Sc2cccc3c(NC(=O)C(C)(C)C)nccc23)cc1</smiles>
The amide was obtained as yellow solid, $92.4 \mathrm{mg}, 88 \%$ yield. Mp: $155-157{ }^{\circ} \mathrm{C}$ ${ }^{1} \mathrm{H}$ NMR (400 MHz, $\left.\mathrm{CDCl}_{3}\right): \delta 10.36(\mathrm{~s}, 1 \mathrm{H}), 8.82(\mathrm{~d}, J=4.2 \mathrm{~Hz}, 1 \mathrm{H}), 8.78(\mathrm{~d}$, $J=8.1 \mathrm{~Hz}, 1 \mathrm{H}), 8.70(\mathrm{~d}, J=8.5 \mathrm{~Hz}, 1 \mathrm{H}), 7.82(\mathrm{~d}, J=8.1 \mathrm{~Hz}, 1 \mathrm{H}), 7.47(\mathrm{dd}, J$ $=8.5,4.2 \mathrm{~Hz}, 1 \mathrm{H}), 7.00(\mathrm{~s}, 4 \mathrm{H}), 2.26(\mathrm{~s}, 3 \mathrm{H}), 1.44(\mathrm{~s}, 9 \mathrm{H}) .{ }^{13} \mathrm{C}$ NMR $(101$ $\left.\mathrm{MHz}, \mathrm{CDCl}_{3}\right): \delta 177.4,148.3,139.4,135.9,135.8,135.4,135.0,133.8,129.8$, $129.1,128.2,123.6,122.2,116.3,40.4,27.7,20.9$. HRMS m/z (EI) calcd for $\left[\mathrm{C}_{21} \mathrm{H}_{22} \mathrm{~N}_{2} \mathrm{OS}\right]: 350.1453$, found: 350.1450 .

$N$-(5-((4-methoxyphenyl)thio)quinolin-8-yl)pivalamide (3c)

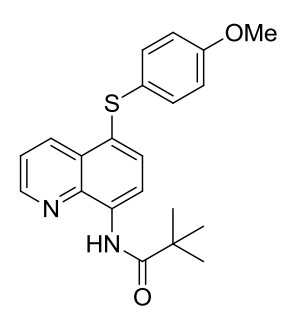

The amide was obtained as yellow oil, $102.1 \mathrm{mg}, 93 \%$ yield. ${ }^{1} \mathrm{H}$ NMR (400 $\left.\mathrm{MHz}, \mathrm{CDCl}_{3}\right): \delta 10.32(\mathrm{~s}, 1 \mathrm{H}), 8.81(\mathrm{dd}, J=4.1,1.2 \mathrm{~Hz}, 1 \mathrm{H}), 8.74(\mathrm{~d}, J=$ $8.1 \mathrm{~Hz}, 1 \mathrm{H}), 8.69(\mathrm{dd}, J=8.5,1.3 \mathrm{~Hz}, 1 \mathrm{H}), 7.69(\mathrm{~d}, J=8.1 \mathrm{~Hz}, 1 \mathrm{H}), 7.46$ (dd, $J=8.5,4.2 \mathrm{~Hz}, 1 \mathrm{H}), 7.16(\mathrm{~d}, J=8.8 \mathrm{~Hz}, 2 \mathrm{H}), 6.77(\mathrm{~d}, J=8.8 \mathrm{~Hz}, 2 \mathrm{H})$, $3.74(\mathrm{~s}, 3 \mathrm{H}), 1.43$ (s, 9H). ${ }^{13} \mathrm{C}$ NMR $\left(101 \mathrm{MHz}, \mathrm{CDCl}_{3}\right): \delta$ 177.2, 158.6, $148.3,139.3,135.19,134.5,133.7,131.2,128.4,127.0,125.2,122.0,116.0$,

114.8, 55.2, 40.3, 27.6. HRMS m/z (EI) calcd for $\left[\mathrm{C}_{21} \mathrm{H}_{22} \mathrm{~N}_{2} \mathrm{O}_{2} \mathrm{~S}\right]: 366.1402$, found: 366.1406 .

$N$-(5-((4-chlorophenyl)thio)quinolin-8-yl)pivalamide (3d)

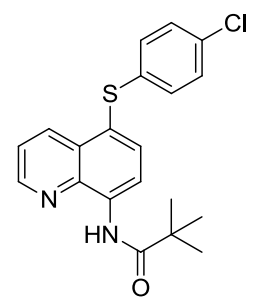

The amide was obtained as yellow solid, $91.3 \mathrm{mg}, 82 \%$ yield. Mp: 147-149 ${ }^{\circ} \mathrm{C} .{ }^{1} \mathrm{H}$ NMR (400 MHz, $\left.\mathrm{CDCl}_{3}\right): \delta 10.39(\mathrm{~s}, 1 \mathrm{H}), 8.82(\mathrm{dd}, J=11.1,6.0 \mathrm{~Hz}$, $2 \mathrm{H}), 8.61(\mathrm{~d}, J=8.4 \mathrm{~Hz}, 1 \mathrm{H}), 7.88(\mathrm{~d}, J=8.1 \mathrm{~Hz}, 1 \mathrm{H}), 7.48(\mathrm{dd}, J=8.5,4.2$ $\mathrm{Hz}, 1 \mathrm{H}), 7.12(\mathrm{~d}, J=8.5 \mathrm{~Hz}, 2 \mathrm{H}), 6.91(\mathrm{dd}, J=28.5,8.5 \mathrm{~Hz}, 2 \mathrm{H}), 1.44(\mathrm{~s}$, $9 \mathrm{H}) .{ }^{13} \mathrm{C}$ NMR $\left(101 \mathrm{MHz}, \mathrm{CDCl}_{3}\right): \delta 177.5,148.5,139.6,136.6,136.6$, 134.7, 132.0, 131.5, 129.2, 129.1, 128.4, 122.5, 121.6, 116.0, 40.4, 27.7. HRMS m/z (EI) calcd for [ $\left.\mathrm{C}_{20} \mathrm{H}_{19} \mathrm{ClN}_{2} \mathrm{OS}\right]: 370.0907$, found: 370.0904 .

$N$-(5-((4-bromophenyl)thio)quinolin-8-yl)pivalamide (3e)

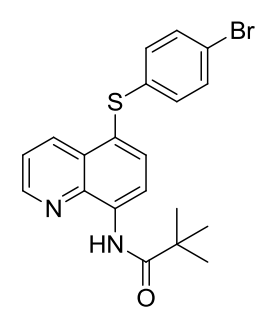

The amide was obtained as yellow solid, $100.8 \mathrm{mg}, 81 \%$ yield. Mp: 170-172 ${ }^{\circ} \mathrm{C} .{ }^{1} \mathrm{H}$ NMR $\left(400 \mathrm{MHz}, \mathrm{CDCl}_{3}\right): \delta 10.39(\mathrm{~s}, 1 \mathrm{H}), 8.88-8.76(\mathrm{~m}, 2 \mathrm{H}), 8.61$ $(\mathrm{dd}, J=8.5,1.1 \mathrm{~Hz}, 1 \mathrm{H}), 7.89(\mathrm{~d}, J=8.1 \mathrm{~Hz}, 1 \mathrm{H}), 7.48(\mathrm{dd}, J=8.5,4.2 \mathrm{~Hz}$, $1 \mathrm{H}), 7.30-7.22(\mathrm{~m}, 2 \mathrm{H}), 6.88(\mathrm{~d}, J=8.5 \mathrm{~Hz}, 2 \mathrm{H}), 1.44(\mathrm{~s}, 9 \mathrm{H}) .{ }^{13} \mathrm{C} \mathrm{NMR}$ $\left(101 \mathrm{MHz} \mathrm{CDCl}_{3}\right): \delta 177.5,148.5,139.6,137.4,136.8,136.6,134.7,132.0$, 
129.2, 128.5, 122.5, 121.4, 119.3, 116.0, 40.5, 27.7. HRMS m/z (EI) calcd for $\left[\mathrm{C}_{20} \mathrm{H}_{19} \mathrm{BrN}_{2} \mathrm{OS}\right]$ : 414.0401, found: 414.0404.

$N$-(5-((4-nitrophenyl)thio)quinolin-8-yl)pivalamide (3f)

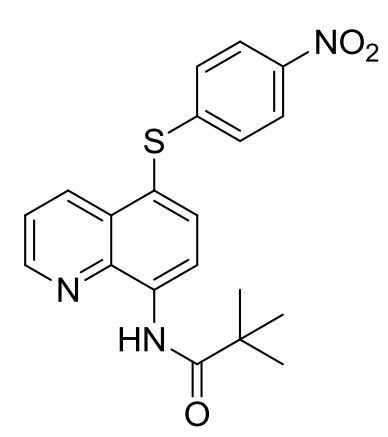

The amide was obtained as yellow solid, $89.1 \mathrm{mg}, 78 \%$ yield. Mp: 169-170 ${ }^{\circ} \mathrm{C} .{ }^{1} \mathrm{H}$ NMR (400 MHz, $\mathrm{CDCl}_{3}$ ): $\delta 10.43$ (s, 1H), 8.86 (dd, $J=4.6,3.4 \mathrm{~Hz}, 2 \mathrm{H}), 8.52(\mathrm{~d}, J=8.5 \mathrm{~Hz}, 1 \mathrm{H}), 7.95(\mathrm{dd}, J=8.4,3.5$ $\mathrm{Hz}, 3 \mathrm{H}), 7.50(\mathrm{dd}, J=8.5,4.2 \mathrm{~Hz}, 1 \mathrm{H}), 6.99(\mathrm{~d}, J=8.9 \mathrm{~Hz}, 2 \mathrm{H})$, $1.44(\mathrm{~s}, 9 \mathrm{H}) .{ }^{13} \mathrm{C}$ NMR $\left(101 \mathrm{MHz}, \mathrm{CDCl}_{3}\right): \delta 177.5,148.8,148.6$, $145.2,139.6,138.0,137.6,134.5,129.4,125.6,124.1,123.0,118.6$, 116.1, 40.5, 27.6. HRMS $\mathrm{m} / \mathrm{z}$ (EI) calcd for $\left[\mathrm{C}_{20} \mathrm{H}_{19} \mathrm{~N}_{3} \mathrm{O}_{3} \mathrm{~S}\right]$ : 381.1147, found: 381.1149. Since the polarity and $R_{f}$ value of $\mathbf{3 f}$ and starting material 1,2-bis(4-nitrophenyl)disulfane almost the same, it's very difficult to remove 1,2-bis(4-nitrophenyl)disulfane from the reaction system. Inpurity peaks: ${ }^{1} H N M R \delta 8.20,7.20$ and ${ }^{13}$ CNMR: $\delta$ 126.30, 124.30 belong to 1,2-bis(4-nitrophenyl)disulfane.

$N$-(5-((3-nitrophenyl)thio)quinolin-8-yl)pivalamide (3g)

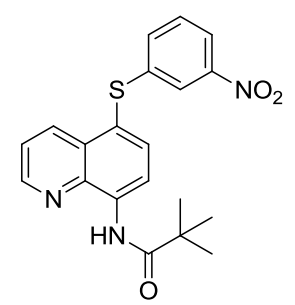

The amide was obtained as yellow solid, $88.0 \mathrm{mg}$, 77\% yield. Mp: 154-157 ${ }^{\circ} \mathrm{C} .{ }^{1} \mathrm{H}$ NMR $\left(400 \mathrm{MHz}, \mathrm{CDCl}_{3}\right): \delta 10.43(\mathrm{~s}, 1 \mathrm{H}), 8.87(\mathrm{~d}, J=6.4 \mathrm{~Hz}, 2 \mathrm{H})$, $8.59(\mathrm{~d}, J=8.5 \mathrm{~Hz}, 1 \mathrm{H}), 8.01-7.82(\mathrm{~m}, 2 \mathrm{H}), 7.51(\mathrm{dd}, J=8.5,4.2 \mathrm{~Hz}, 1 \mathrm{H})$, $7.36-7.18(\mathrm{~m}, 3 \mathrm{H}), 1.45(\mathrm{~s}, 9 \mathrm{H}) .{ }^{13} \mathrm{C} \mathrm{NMR}\left(101 \mathrm{MHz}, \mathrm{CDCl}_{3}\right): \delta 177.6$, $149.4,148.7,141.5,139.6,137.8,137.3,136.2$, 134.4, 131.9, 129.6, 129.3, $122.8,120.9,120.2,116.11,40.5,27.7$. HRMS $\mathrm{m} / \mathrm{z}$ (EI) calcd for $\left[\mathrm{C}_{20} \mathrm{H}_{19} \mathrm{~N}_{3} \mathrm{O}_{3} \mathrm{~S}\right]: 381.1147$, found: 381.1145 .

$N$-(5-((2-nitrophenyl)thio)quinolin-8-yl)pivalamide (3h)

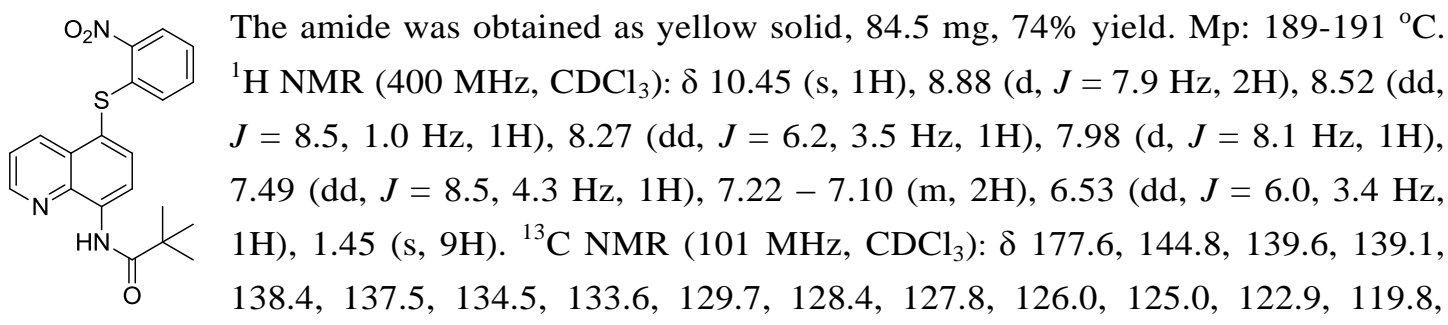

116.3, 40.5, 27.7. HRMS m/z (EI) calcd for $\left[\mathrm{C}_{20} \mathrm{H}_{19} \mathrm{~N}_{3} \mathrm{O}_{3} \mathrm{~S}\right]$ : 381.1147 , found: 381.1150 .

$N$-(5-((4-(tert-butyl)phenyl)thio)quinolin-8-yl)pivalamide (3i)

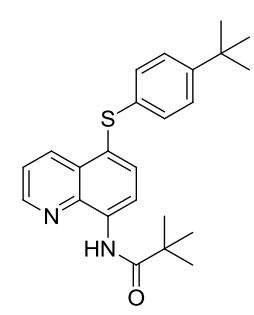

The amide was obtained as yellow oil, $82.3 \mathrm{mg}, 70 \%$ yield. ${ }^{1} \mathrm{H}$ NMR (400 $\left.\mathrm{MHz} \mathrm{CDCl}_{3}\right) \delta 10.38(\mathrm{~s}, 1 \mathrm{H}), 8.81(\mathrm{dd}, J=13.5,6.1 \mathrm{~Hz}, 2 \mathrm{H}), 8.72(\mathrm{~d}, J=8.5$ $\mathrm{Hz}, 1 \mathrm{H}), 7.85(\mathrm{~d}, J=8.1 \mathrm{~Hz}, 1 \mathrm{H}), 7.47(\mathrm{dd}, J=8.5,4.2 \mathrm{~Hz}, 1 \mathrm{H}), 7.20(\mathrm{~d}, J=$ $8.4 \mathrm{~Hz}, 2 \mathrm{H}), 7.02$ (d, $J=8.3 \mathrm{~Hz}, 2 \mathrm{H}), 1.44(\mathrm{~s}, 9 \mathrm{H}), 1.25$ (s, 9H). ${ }^{13} \mathrm{C} \mathrm{NMR}$ 
$\left(101 \mathrm{MHz} \mathrm{CDCl}_{3}\right): \delta 177.4,149.0,148.4,139.5,135.9,135.8,135.0,134.1,129.3,127.5$, $126.1,123.2,122.3,116.1,40.4,34.3,31.2,27.7$.

$N$-(5-(cyclohexylthio)quinolin-8-yl)pivalamide (3j)

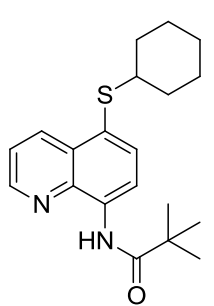

The amide was obtained as yellow solid, $61.5 \mathrm{mg}, 60 \%$ yield. Mp: $139-140{ }^{\circ} \mathrm{C}$. ${ }^{1} \mathrm{H}$ NMR (400 MHz, $\left.\mathrm{CDCl}_{3}\right): \delta 10.33(\mathrm{~s}, 1 \mathrm{H}), 8.89(\mathrm{dd}, J=8.5,1.4 \mathrm{~Hz}, 1 \mathrm{H})$, $8.84-8.81(\mathrm{~m}, 1 \mathrm{H}), 8.72(\mathrm{dd}, J=16.4,8.2 \mathrm{~Hz}, 1 \mathrm{H}), 7.79(\mathrm{~d}, J=8.1 \mathrm{~Hz}, 1 \mathrm{H})$, $7.53(\mathrm{dd}, J=8.5,4.2 \mathrm{~Hz}, 1 \mathrm{H}), 2.93(\mathrm{ddd}, J=10.7,7.0,3.7 \mathrm{~Hz}, 1 \mathrm{H}), 1.88(\mathrm{~d}, J=$ $13.5 \mathrm{~Hz}, 2 \mathrm{H}), 1.72(\mathrm{dd}, J=8.6,4.5 \mathrm{~Hz}, 2 \mathrm{H}), 1.42(\mathrm{~s}, 9 \mathrm{H}), 1.34(\mathrm{dd}, J=22.1$, $11.5 \mathrm{~Hz}, 2 \mathrm{H}), 1.22(\mathrm{dd}, J=17.2,7.9 \mathrm{~Hz}, 4 \mathrm{H}) .{ }^{13} \mathrm{C} \mathrm{NMR}\left(101 \mathrm{MHz}, \mathrm{CDCl}_{3}\right): \delta$ $177.3,148.1$, 139.3, 135.4, 135.2, 135.1, 130.2, 124.5, 121.8, 115.8, 48.5, 40.4, 33.5, 27.7, 26.0, 25.7. HRMS m/z (EI) calcd for $\left[\mathrm{C}_{20} \mathrm{H}_{26} \mathrm{~N}_{2} \mathrm{OS}\right]: 342.1766$, found: 342.1769 .

$N$-(5-(propylthio)quinolin-8-yl)pivalamide (3k)

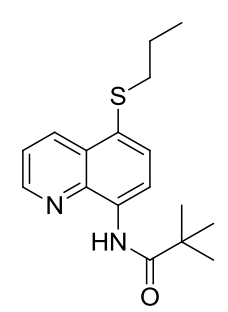

The amide was obtained as yellow oil, $42.6 \mathrm{mg}, 47 \%$ yield. ${ }^{1} \mathrm{H}$ NMR (400 MHz, $\left.\mathrm{CDCl}_{3}\right): \delta 10.30(\mathrm{~s}, 1 \mathrm{H}), 8.85-8.77(\mathrm{~m}, 2 \mathrm{H}), 8.73(\mathrm{~d}, J=8.1 \mathrm{~Hz}, 1 \mathrm{H}), 7.70(\mathrm{~d}$, $J=8.1 \mathrm{~Hz}, 1 \mathrm{H}), 7.52(\mathrm{dd}, J=8.5,4.2 \mathrm{~Hz}, 1 \mathrm{H}), 2.84(\mathrm{t}, J=7.3 \mathrm{~Hz}, 2 \mathrm{H}), 1.66-$ $1.54(\mathrm{~m}, 2 \mathrm{H}), 1.42(\mathrm{~s}, 9 \mathrm{H}), 0.98(\mathrm{t}, J=7.3 \mathrm{~Hz}, 3 \mathrm{H}) .{ }^{13} \mathrm{C}$ NMR (101 MHz, $\left.\mathrm{CDCl}_{3}\right): \delta 177.2,148.2,139.3,134.5,134.4,132.2,128.8,126.1,121.7,116.0$, 40.4, 37.9, 27.7, 22.7, 13.3. HRMS m/z (EI) calcd for $\left[\mathrm{C}_{17} \mathrm{H}_{22} \mathrm{~N}_{2} \mathrm{OS}\right]$ : 302.1453 , found: 302.1450 .

$N$-(5-(phenylthio)quinolin-8-yl)acetamide (4a)

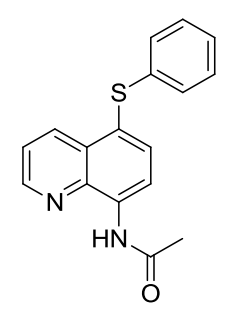

The amide was obtained as yellow solid, $66.1 \mathrm{mg}, 75 \%$ yield. Mp: $156-157{ }^{\circ} \mathrm{C}$. ${ }^{1} \mathrm{H}$ NMR (400 MHz, $\mathrm{CDCl}_{3}$ ): $\delta 9.89(\mathrm{~s}, 1 \mathrm{H}), 8.78(\mathrm{dd}, J=17.3,5.4 \mathrm{~Hz}, 2 \mathrm{H})$, $8.67(\mathrm{~d}, J=8.5 \mathrm{~Hz}, 1 \mathrm{H}), 7.86(\mathrm{~d}, J=8.1 \mathrm{~Hz}, 1 \mathrm{H}), 7.46(\mathrm{dd}, J=8.5,4.2 \mathrm{~Hz}, 1 \mathrm{H})$, $7.18(\mathrm{t}, J=7.4 \mathrm{~Hz}, 2 \mathrm{H}), 7.09(\mathrm{dd}, J=18.9,7.6 \mathrm{~Hz}, 3 \mathrm{H}), 2.37(\mathrm{~s}, 3 \mathrm{H}) .{ }^{13} \mathrm{C} \mathrm{NMR}$ $\left(101 \mathrm{MHz}, \mathrm{CDCl}_{3}\right): \delta 168.9,148.3,139.0,137.7,136.0,135.9,134.9,129.3$, $129.0,127.5,125.8,122.8,122.4,116.2,25.1$. HRMS m/z (EI) calcd for $\left[\mathrm{C}_{17} \mathrm{H}_{14} \mathrm{~N}_{2} \mathrm{OS}\right]: 294.0827$, found: 294.0825 .

$N$-(5-(p-tolylthio)quinolin-8-yl)acetamide (4b)

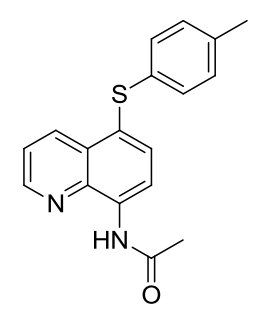

The amide was obtained as yellow solid, $72.1 \mathrm{mg}, 78 \%$ yield. Mp: $163-164{ }^{\circ} \mathrm{C}$. ${ }^{1} \mathrm{H}$ NMR (400 MHz, $\mathrm{CDCl}_{3}$ ): $\delta 9.86(\mathrm{~s}, 1 \mathrm{H}), 8.78(\mathrm{~d}, J=2.7 \mathrm{~Hz}, 1 \mathrm{H}), 8.73$ (d, $J=8.1 \mathrm{~Hz}, 1 \mathrm{H}), 8.67(\mathrm{~d}, J=8.5 \mathrm{~Hz}, 1 \mathrm{H}), 7.78(\mathrm{~d}, J=8.1 \mathrm{~Hz}, 1 \mathrm{H}), 7.45(\mathrm{dd}, J$ $=8.5,4.1 \mathrm{~Hz}, 1 \mathrm{H}), 7.06-6.96(\mathrm{~m}, 4 \mathrm{H}), 2.36(\mathrm{~s}, 3 \mathrm{H}), 2.26(\mathrm{~s}, 3 \mathrm{H}) .{ }^{13} \mathrm{C} \mathrm{NMR}$ $\left(101 \mathrm{MHz}, \mathrm{CDCl}_{3}\right): \delta 168.8,148.2,138.9,136.0,135.5,135.0,134.8,133.5$, 129.8, 128.9, 128.4, 123.9, 122.2, 116.2, 25.1, 20.9. HRMS m/z (EI) calcd for [ $\left.\mathrm{C}_{18} \mathrm{H}_{16} \mathrm{~N}_{2} \mathrm{OS}\right]:$ 308.0983, found: 308.0987 . 
$N$-(5-((4-methoxyphenyl)thio)quinolin-8-yl)acetamide (4c)

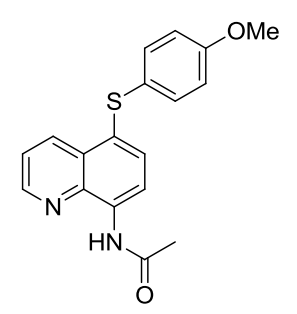

The amide was obtained as yellow solid, $77.8 \mathrm{mg}, 80 \%$ yield. Mp: $151-153$ ${ }^{\circ} \mathrm{C} .{ }^{1} \mathrm{H}$ NMR $\left(400 \mathrm{MHz}, \mathrm{CDCl}_{3}\right): \delta 9.83(\mathrm{~s}, 1 \mathrm{H}), 8.80(\mathrm{~d}, J=4.0 \mathrm{~Hz}, 1 \mathrm{H})$, $8.72-8.67(\mathrm{~m}, 2 \mathrm{H}), 7.65(\mathrm{~d}, J=8.1 \mathrm{~Hz}, 1 \mathrm{H}), 7.48(\mathrm{dd}, J=8.4,4.1 \mathrm{~Hz}, 1 \mathrm{H})$, 7.19 (d, $J=8.3 \mathrm{~Hz}, 2 \mathrm{H}), 6.79$ (d, $J=8.3 \mathrm{~Hz}, 2 \mathrm{H}), 3.76$ (s, 3H), 2.35 (s, 3H). ${ }^{13} \mathrm{C}$ NMR $\left(101 \mathrm{MHz}, \mathrm{CDCl}_{3}\right): \delta 168.8,158.9,148.2,138.9,134.9,134.5$, 133.2, 131.7, 128.3, 126.7, 125.8, 122.1, 116.3, 114.9, 55.3, 25.1. HRMS $\mathrm{m} / \mathrm{z}$ (EI) calcd for [ $\left.\mathrm{C}_{18} \mathrm{H}_{16} \mathrm{~N}_{2} \mathrm{O}_{2} \mathrm{~S}\right]: 324.0932$, found: 324.0940 .

$N$-(5-((4-chlorophenyl)thio)quinolin-8-yl)acetamide (4d)<smiles>CC(=O)Nc1ccc(Sc2ccc(Cl)cc2)c2cccnc12</smiles>

The amide was obtained as yellow solid, $70.8 \mathrm{mg}, 72 \%$ yield. Mp: $148-150$ ${ }^{\circ} \mathrm{C} .{ }^{1} \mathrm{H}$ NMR (400 MHz, $\mathrm{CDCl}_{3}$ ): $\delta 9.90(\mathrm{~s}, 1 \mathrm{H}), 8.81(\mathrm{~d}, J=2.6 \mathrm{~Hz}, 1 \mathrm{H})$, $8.77(\mathrm{~d}, J=8.1 \mathrm{~Hz}, 1 \mathrm{H}), 8.61(\mathrm{~d}, J=8.5 \mathrm{~Hz}, 1 \mathrm{H}), 7.86(\mathrm{~d}, J=8.1 \mathrm{~Hz}, 1 \mathrm{H})$, $7.48(\mathrm{dd}, J=8.5,4.2 \mathrm{~Hz}, 1 \mathrm{H}), 7.13(\mathrm{~d}, J=8.6 \mathrm{~Hz}, 2 \mathrm{H}), 6.97(\mathrm{~d}, J=8.5 \mathrm{~Hz}$, $2 \mathrm{H}), 2.38(\mathrm{~s}, 3 \mathrm{H}) .{ }^{13} \mathrm{C} \mathrm{NMR}\left(101 \mathrm{MHz}, \mathrm{CDCl}_{3}\right): \delta 168.9,148.4,139.0,136.3$, 136.2, 134.7, 133.4, 131.6, 129.1, 128.5, 122.5, 122.1, 116.3, 116.2, 25.1. HRMS m/z (EI) calcd for [ $\left.\mathrm{C}_{17} \mathrm{H}_{13} \mathrm{ClN}_{2} \mathrm{OS}\right]$ : 328.0437 , found: 328.0434 .

2,2-dimethyl- $N$-(5-(phenylthio)quinolin-8-yl)butanamide (4e)

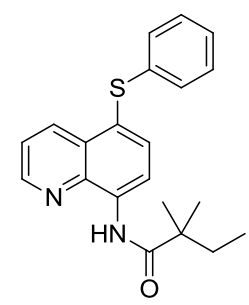

The amide was obtained as white solid, $85.4 \mathrm{mg}, 85 \%$ yield. Mp: $146-147{ }^{\circ} \mathrm{C}$. ${ }^{1} \mathrm{H}$ NMR (400 MHz, $\mathrm{CDCl}_{3}$ ): $\delta 10.36(\mathrm{~s}, 1 \mathrm{H}), 8.90-8.74(\mathrm{~m}, 2 \mathrm{H}), 8.66(\mathrm{~d}, J$ $=8.5 \mathrm{~Hz}, 1 \mathrm{H}), 7.88(\mathrm{~d}, J=8.1 \mathrm{~Hz}, 1 \mathrm{H}), 7.45(\mathrm{dd}, J=8.4,4.1 \mathrm{~Hz}, 1 \mathrm{H}), 7.16(\mathrm{t}$, $J=7.6 \mathrm{~Hz}, 2 \mathrm{H}), 7.07(\mathrm{dd}, J=15.1,7.4 \mathrm{~Hz}, 3 \mathrm{H}), 1.93-1.67(\mathrm{~m}, 2 \mathrm{H}), 1.41(\mathrm{~s}$, $6 \mathrm{H}), 0.98(\mathrm{t}, J=7.4 \mathrm{~Hz}, 3 \mathrm{H}) .{ }^{13} \mathrm{C}$ NMR $\left(101 \mathrm{MHz}, \mathrm{CDCl}_{3}\right): \delta 176.8,148.4$, $139.5,137.9,136.3,136.2,134.9,129.3,128.9,127.2,125.6,122.3,122.3$, 116.0, 44.1, 34.0, 25.0, 9.3. HRMS $\mathrm{m} / \mathrm{z}$ (EI) calcd for $\left[\mathrm{C}_{21} \mathrm{H}_{22} \mathrm{~N}_{2} \mathrm{OS}\right]: 350.1453$, found: 350.1455 .

2,2-dimethyl- $N$-(5-( $p$-tolylthio)quinolin-8-yl)butanamide (4f)

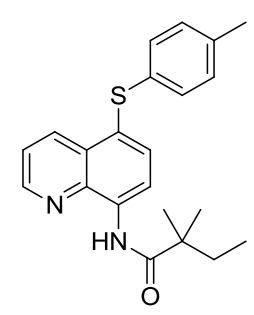

The amide was obtained as yellow solid, $93.9 \mathrm{mg}, 86 \%$ yield. Mp: $154-156{ }^{\circ} \mathrm{C}$. ${ }^{1} \mathrm{H}$ NMR (400 MHz, $\left.\mathrm{CDCl}_{3}\right): \delta 10.34(\mathrm{~s}, 1 \mathrm{H}), 8.81(\mathrm{t}, J=6.1 \mathrm{~Hz}, 2 \mathrm{H}), 8.68(\mathrm{~d}$, $J=8.5 \mathrm{~Hz}, 1 \mathrm{H}), 7.82(\mathrm{~d}, J=8.1 \mathrm{~Hz}, 1 \mathrm{H}), 7.45(\mathrm{dd}, J=7.9,3.5 \mathrm{~Hz}, 1 \mathrm{H}), 7.06$ $-6.93(\mathrm{~m}, 4 \mathrm{H}), 2.25$ (s, 3H), $1.87-1.74(\mathrm{~m}, 2 \mathrm{H}), 1.40(\mathrm{~s}, 6 \mathrm{H}), 0.97$ (t, $J=7.4$ $\mathrm{Hz}, 3 \mathrm{H}) .{ }^{13} \mathrm{C}$ NMR $\left(101 \mathrm{MHz}, \mathrm{CDCl}_{3}\right): \delta 176.7,148.3,139.4,135.8,135.8$, 135.4, 134.8, 133.8, 129.8, 129.0, 128.1, 123.3, 122.2, 116.0, 44.1, 34.0, 25.0,

20.9, 9.3. HRMS m/z (EI) calcd for [ $\mathrm{C}_{22} \mathrm{H}_{24} \mathrm{~N}_{2} \mathrm{OS}$ ]: 364.1609 , found: 364.1605 . 


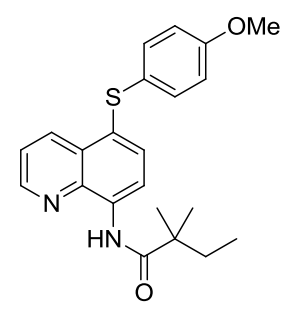

The amide was obtained as yellow oil, $100.3 \mathrm{mg}, 88 \%$ yield. ${ }^{1} \mathrm{H}$ NMR (400 $\left.\mathrm{MHz}, \mathrm{CDCl}_{3}\right): \delta 10.30(\mathrm{~s}, 1 \mathrm{H}), 8.78(\mathrm{dd}, J=11.6,6.1 \mathrm{~Hz}, 2 \mathrm{H}), 8.68(\mathrm{~d}, J=$ $8.4 \mathrm{~Hz}, 1 \mathrm{H}), 7.69$ (d, $J=8.1 \mathrm{~Hz}, 1 \mathrm{H}), 7.43(\mathrm{dd}, J=8.5,4.2 \mathrm{~Hz}, 1 \mathrm{H}), 7.16$ $(\mathrm{d}, J=8.7 \mathrm{~Hz}, 2 \mathrm{H}), 6.76(\mathrm{~d}, J=8.7 \mathrm{~Hz}, 2 \mathrm{H}), 3.72(\mathrm{~s}, 3 \mathrm{H}), 1.76(\mathrm{q}, J=7.5$ $\mathrm{Hz}, 2 \mathrm{H}), 1.39(\mathrm{~s}, 6 \mathrm{H}), 0.95(\mathrm{t}, J=7.5 \mathrm{~Hz}, 3 \mathrm{H}) .{ }^{13} \mathrm{C}$ NMR (101 MHz, $\left.\mathrm{CDCl}_{3}\right): \delta 176.5,158.6,148.2,139.3,135.1,134.4,133.6,131.2,128.4$, $126.9,125.1,121.9,115.9,114.7,55.2,44.0,34.0,25.0,9.2$. HRMS m/z (EI) calcd for $\left[\mathrm{C}_{22} \mathrm{H}_{24} \mathrm{~N}_{2} \mathrm{O}_{2} \mathrm{~S}\right]: 380.1558$, found: 380.1556 .

$N$-(5-((4-chlorophenyl)thio)quinolin-8-yl)-2,2-dimethylbutanamide (4h)

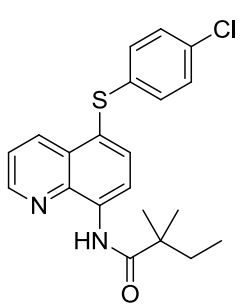

The amide was obtained as yellow solid, $89.8 \mathrm{mg}, 78 \%$ yield. Mp: 138-139 ${ }^{\circ} \mathrm{C} .{ }^{1} \mathrm{H}$ NMR $\left(400 \mathrm{MHz}, \mathrm{CDCl}_{3}\right): \delta 10.35(\mathrm{~s}, 1 \mathrm{H}), 8.91-8.76(\mathrm{~m}, 2 \mathrm{H}), 8.61(\mathrm{~d}$, $J=7.9 \mathrm{~Hz}, 1 \mathrm{H}), 7.88(\mathrm{~d}, J=8.1 \mathrm{~Hz}, 1 \mathrm{H}), 7.48(\mathrm{dd}, J=8.5,4.2 \mathrm{~Hz}, 1 \mathrm{H}), 7.13$ $(\mathrm{d}, J=8.5 \mathrm{~Hz}, 2 \mathrm{H}), 6.96(\mathrm{~d}, J=8.5 \mathrm{~Hz}, 2 \mathrm{H}), 1.78(\mathrm{q}, J=7.5 \mathrm{~Hz}, 2 \mathrm{H}), 1.40(\mathrm{~s}$, $6 \mathrm{H}), 0.97(\mathrm{t}, J=7.5 \mathrm{~Hz}, 3 \mathrm{H}) .{ }^{13} \mathrm{C} \mathrm{NMR}\left(101 \mathrm{MHz}, \mathrm{CDCl}_{3}\right): \delta 176.9,148.5$, $139.6,136.7,136.5,134.7,132.0,131.5,129.2,129.1,128.4,122.5,121.6$, 116.0, 44.2, 34.1, 25.1, 9.3. HRMS m/z (EI) calcd for $\left[\mathrm{C}_{21} \mathrm{H}_{21} \mathrm{ClN}_{2} \mathrm{OS}\right]$ : 384.1063, found: 384.1067 .

2-ethyl- $N$-(5-(phenylthio)quinolin-8-yl)butanamide (4i)

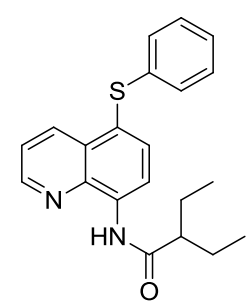

The amide was obtained as yellow solid, $88.2 \mathrm{mg}, 84 \%$ yield. Mp: $146-148{ }^{\circ} \mathrm{C}$. ${ }^{1} \mathrm{H}$ NMR (400 MHz, $\left.\mathrm{CDCl}_{3}\right): \delta 9.97(\mathrm{~s}, 1 \mathrm{H}), 8.84(\mathrm{dd}, J=12.6,6.1 \mathrm{~Hz}, 2 \mathrm{H})$, $8.67(\mathrm{~d}, J=8.5 \mathrm{~Hz}, 1 \mathrm{H}), 7.88(\mathrm{~d}, J=8.1 \mathrm{~Hz}, 1 \mathrm{H}), 7.46(\mathrm{dd}, J=8.5,4.2 \mathrm{~Hz}$, $1 \mathrm{H}), 7.17(\mathrm{t}, J=7.4 \mathrm{~Hz}, 2 \mathrm{H}), 7.08(\mathrm{dd}, J=14.2,7.5 \mathrm{~Hz}, 3 \mathrm{H}), 2.41-2.28(\mathrm{~m}$, $1 \mathrm{H}), 1.91-1.74(\mathrm{~m}, 2 \mathrm{H}), 1.74-1.60(\mathrm{~m}, 2 \mathrm{H}), 1.02(\mathrm{t}, J=7.4 \mathrm{~Hz}, 6 \mathrm{H}) .{ }^{13} \mathrm{C}$ NMR (101 MHz, $\left.\mathrm{CDCl}_{3}\right): \delta 174.9,148.3,139.2,137.8,136.2,136.0,134.9$, $129.3,129.0,127.4,125.7,122.5,122.3,116.2,52.6,25.8,12.1$. HRMS m/z (EI) calcd for $\left[\mathrm{C}_{21} \mathrm{H}_{22} \mathrm{~N}_{2} \mathrm{OS}\right]: 350.1453$, found: 350.1455 .

$N$-(5-(phenylthio)quinolin-8-yl)cyclopentanecarboxamide (4j)

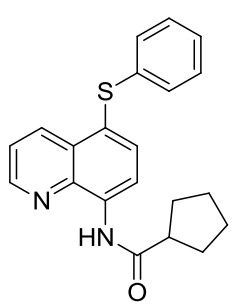

The amide was obtained as yellow oil, $75.2 \mathrm{mg}, 72 \%$ yield. ${ }^{1} \mathrm{H}$ NMR (400 $\left.\mathrm{MHz} \mathrm{CDCl}_{3}\right): \delta 9.98(\mathrm{~s}, 1 \mathrm{H}), 8.80(\mathrm{~d}, J=8.0 \mathrm{~Hz}, 2 \mathrm{H}), 8.67(\mathrm{~d}, J=8.5 \mathrm{~Hz}$, $1 \mathrm{H}), 7.88(\mathrm{~d}, J=8.1 \mathrm{~Hz}, 1 \mathrm{H}), 7.46(\mathrm{dd}, J=8.5,4.2 \mathrm{~Hz}, 1 \mathrm{H}), 7.17(\mathrm{t}, J=7.4$ $\mathrm{Hz}, 2 \mathrm{H}), 7.12-7.02(\mathrm{~m}, 3 \mathrm{H}), 3.06-2.87(\mathrm{~m}, 1 \mathrm{H}), 2.08-1.97(\mathrm{~m}, 4 \mathrm{H}), 1.90-$ $1.80(\mathrm{~m}, 2 \mathrm{H}), 1.75-1.65(\mathrm{~m}, 2 \mathrm{H}) .{ }^{13} \mathrm{C} \mathrm{NMR}\left(101 \mathrm{MHz}, \mathrm{CDCl}_{3}\right): \delta 175.2$, $148.3,139.2,137.9,136.3,136.3,134.9,129.4,129.0,127.3,125.7,122.4$, 122.3, 116.2, 47.4, 30.6, 26.0. HRMS m/z (EI) calcd for $\left[\mathrm{C}_{21} \mathrm{H}_{20} \mathrm{~N}_{2} \mathrm{OS}\right]$ : 348.1296, found: 348.1292 . 


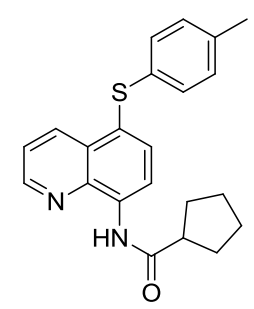

The amide was obtained as yellow solid, $78.2 \mathrm{mg}, 72 \%$ yield. Mp: 170-171 ${ }^{\circ} \mathrm{C} .{ }^{1} \mathrm{H}$ NMR $\left(400 \mathrm{MHz}, \mathrm{CDCl}_{3}\right): \delta 9.96(\mathrm{~s}, 1 \mathrm{H}), 8.78(\mathrm{dd}, J=12.9,5.9 \mathrm{~Hz}$, $2 \mathrm{H}), 8.68(\mathrm{~d}, J=8.5 \mathrm{~Hz}, 1 \mathrm{H}), 7.81(\mathrm{~d}, J=8.1 \mathrm{~Hz}, 1 \mathrm{H}), 7.46(\mathrm{dd}, J=8.5,4.2$ $\mathrm{Hz}, 1 \mathrm{H}), 7.00(\mathrm{~s}, 4 \mathrm{H}), 3.00-2.91(\mathrm{~m}, 1 \mathrm{H}), 2.26(\mathrm{~s}, 3 \mathrm{H}), 2.08-1.94(\mathrm{~m}, 4 \mathrm{H})$, $1.91-1.80(\mathrm{~m}, 2 \mathrm{H}), 1.69$ (d, $J=11.8 \mathrm{~Hz}, 2 \mathrm{H}) .{ }^{13} \mathrm{C}$ NMR (101 MHz, $\left.\mathrm{CDCl}_{3}\right)$ : $\delta 175.2,148.2,139.1,135.9,135.8,135.4,134.8,133.8,129.8,129.1,128.1$, 123.4, 122.2, 116.2, 47.4, 30.6, 26.0, 20.9. HRMS m/z (EI) calcd for $\left[\mathrm{C}_{22} \mathrm{H}_{22} \mathrm{~N}_{2} \mathrm{OS}\right]: 362.1453$, found: 362.1456 .

$N$-(5-((4-methoxyphenyl)thio)quinolin-8-yl)cyclopentanecarboxamide (41)

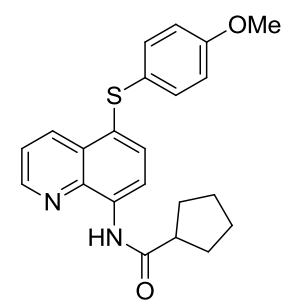

The amide was obtained as yellow solid, $82.7 \mathrm{mg}$, 73\% yield. Mp: 107-108 ${ }^{\circ} \mathrm{C} .{ }^{1} \mathrm{H}$ NMR $\left(400 \mathrm{MHz}, \mathrm{CDCl}_{3}\right): \delta=9.92(\mathrm{~s}, 1 \mathrm{H}), 8.84-8.78(\mathrm{~m}, 1 \mathrm{H}), 8.71$ $(\mathrm{t}, J=9.3 \mathrm{~Hz}, 2 \mathrm{H}), 7.68(\mathrm{~d}, J=8.1 \mathrm{~Hz}, 1 \mathrm{H}), 7.47(\mathrm{dd}, J=8.4,4.1 \mathrm{~Hz}, 1 \mathrm{H})$, $7.16(\mathrm{~d}, J=8.2 \mathrm{~Hz}, 2 \mathrm{H}), 6.78(\mathrm{~d}, J=8.2 \mathrm{~Hz}, 2 \mathrm{H}), 3.75(\mathrm{~s}, 3 \mathrm{H}), 2.99-2.90$ $(\mathrm{m}, 1 \mathrm{H}), 2.12-1.92(\mathrm{~m}, 4 \mathrm{H}), 1.89-1.77(\mathrm{~m}, 2 \mathrm{H}), 1.74-1.60(\mathrm{~m}, 2 \mathrm{H}) .{ }^{13} \mathrm{C}$

NMR (101 MHz, $\left.\mathrm{CDCl}_{3}\right): \delta 175.1,158.7,148.2,139.0,135.2,134.6,133.7$, 131.3, 128.5, 127.0, 125.2, 122.0, 116.2, 114.9, 55.3, 47.4, 30.6, 26.0. HRMS m/z (EI) calcd for $\left[\mathrm{C}_{22} \mathrm{H}_{22} \mathrm{~N}_{2} \mathrm{O}_{2} \mathrm{~S}\right]: 378.1402$, found: 378.1405 .

$N$-(5-((4-chlorophenyl)thio)quinolin-8-yl)cyclopentanecarboxamide (4m)

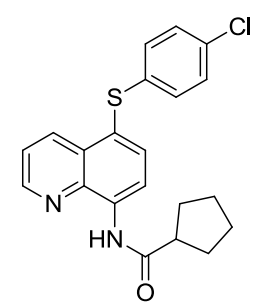

The amide was obtained as yellow solid, $80.2 \mathrm{mg}$, 70\% yield. Mp: 169-171 ${ }^{\circ} \mathrm{C} .{ }^{1} \mathrm{H}$ NMR $\left(400 \mathrm{MHz}, \mathrm{CDCl}_{3}\right): \delta 9.98(\mathrm{~s}, 1 \mathrm{H}), 8.87-8.76(\mathrm{~m}, 2 \mathrm{H}), 8.61(\mathrm{~d}$, $J=8.5 \mathrm{~Hz}, 1 \mathrm{H}), 7.87(\mathrm{~d}, J=8.1 \mathrm{~Hz}, 1 \mathrm{H}), 7.47(\mathrm{dd}, J=8.5,4.2 \mathrm{~Hz}, 1 \mathrm{H}), 7.12$ $(\mathrm{d}, J=8.4 \mathrm{~Hz}, 2 \mathrm{H}), 6.95(\mathrm{~d}, J=8.5 \mathrm{~Hz}, 2 \mathrm{H}), 3.04-2.89(\mathrm{~m}, 1 \mathrm{H}), 2.13-1.95$ $(\mathrm{m}, 4 \mathrm{H}), 1.91-1.80(\mathrm{~m}, 2 \mathrm{H}), 1.75-1.67$ (m, 2H). ${ }^{13} \mathrm{C}$ NMR (101 MHz, $\left.\mathrm{CDCl}_{3}\right): \delta 175.3,148.4,139.2,136.61,136.56,134.8,131.5,129.2,129.1$, 128.4, 122.5, 121.6, 116.2, 47.4, 30.6, 26.0. One signal missing. HRMS m/z (EI) calcd for $\left[\mathrm{C}_{21} \mathrm{H}_{19} \mathrm{ClN}_{2} \mathrm{OS}\right]: 382.0907$, found: 382.0909 .

1-methyl- $N$-(5-(phenylthio)quinolin-8-yl)cyclohexanecarboxamide (4n)

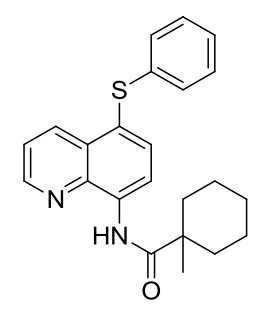

The amide was obtained as yellow solid, $86.8 \mathrm{mg}, 77 \%$ yield. Mp: $112-113{ }^{\circ} \mathrm{C}$. ${ }^{1} \mathrm{H}$ NMR $\left(400 \mathrm{MHz}, \mathrm{CDCl}_{3}\right): \delta 10.40(\mathrm{~s}, 1 \mathrm{H}), 8.88-8.74(\mathrm{~m}, 2 \mathrm{H}), 8.67(\mathrm{~d}, J$ $=8.5 \mathrm{~Hz}, 1 \mathrm{H}), 7.89(\mathrm{~d}, J=8.1 \mathrm{~Hz}, 1 \mathrm{H}), 7.46(\mathrm{dd}, J=8.5,4.2 \mathrm{~Hz}, 1 \mathrm{H}), 7.16(\mathrm{t}$, $J=7.4 \mathrm{~Hz}, 2 \mathrm{H}), 7.07(\mathrm{dd}, J=17.8,7.7 \mathrm{~Hz}, 3 \mathrm{H}), 2.26-2.13(\mathrm{~m}, 2 \mathrm{H}), 1.71-$ $1.49(\mathrm{~m}, 8 \mathrm{H}), 1.37(\mathrm{~s}, 3 \mathrm{H}) .{ }^{13} \mathrm{C} \mathrm{NMR}\left(101 \mathrm{MHz}, \mathrm{CDCl}_{3}\right): \delta 176.8,148.4$, $139.6,138.0,136.4,136.4,134.9,129.4,129.0,127.2,125.6,122.3,122.1$, 116.1, 44.5, 35.8, 26.6, 25.8, 23.0. HRMS m/z (EI) calcd for $\left[\mathrm{C}_{23} \mathrm{H}_{24} \mathrm{~N}_{2} \mathrm{OS}\right]: 376.1609$, found: 376.1612 . 


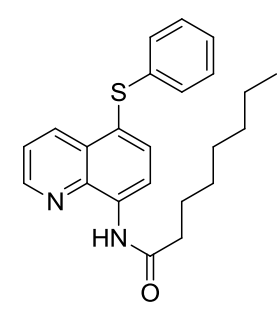

The amide was obtained as yellow solid, $92.9 \mathrm{mg}, 82 \%$ yield. Mp: $94-96{ }^{\circ} \mathrm{C}$. ${ }^{1} \mathrm{H}$ NMR (400 MHz, $\left.\mathrm{CDCl}_{3}\right): \delta 9.91(\mathrm{~s}, 1 \mathrm{H}), 8.80(\mathrm{t}, J=6.6 \mathrm{~Hz}, 2 \mathrm{H}), 8.67$ $(\mathrm{d}, J=8.5 \mathrm{~Hz}, 1 \mathrm{H}), 7.87(\mathrm{~d}, J=8.1 \mathrm{~Hz}, 1 \mathrm{H}), 7.46(\mathrm{dd}, J=8.5,4.2 \mathrm{~Hz}, 1 \mathrm{H})$, $7.17(\mathrm{t}, J=7.4 \mathrm{~Hz}, 2 \mathrm{H}), 7.08(\mathrm{dd}, J=18.4,7.3 \mathrm{~Hz}, 3 \mathrm{H}), 2.58(\mathrm{t}, J=7.6 \mathrm{~Hz}$, $2 \mathrm{H}), 1.89-1.76(\mathrm{~m}, 2 \mathrm{H}), 1.49-1.28(\mathrm{~m}, 8 \mathrm{H}), 0.89(\mathrm{t}, J=6.7 \mathrm{~Hz}, 3 \mathrm{H}) .{ }^{13} \mathrm{C}$ NMR (101 MHz, $\left.\mathrm{CDCl}_{3}\right): \delta 172.1,148.3,139.1,137.8,136.2,136.0,134.9$, 129.3, 129.0, 127.4, 125.7, 122.6, 122.4, 116.2, 38.3, 31.7, 29.2, 29.0, 25.6, 22.6, 14.1. HRMS $\mathrm{m} / \mathrm{z}$ (EI) calcd for $\left[\mathrm{C}_{23} \mathrm{H}_{26} \mathrm{~N}_{2} \mathrm{OS}\right]: 378.1766$, found: 378.1769 .

11-(phenylthio)- $N$-(5-(phenylthio)quinolin-8-yl)undecanamide (4p)

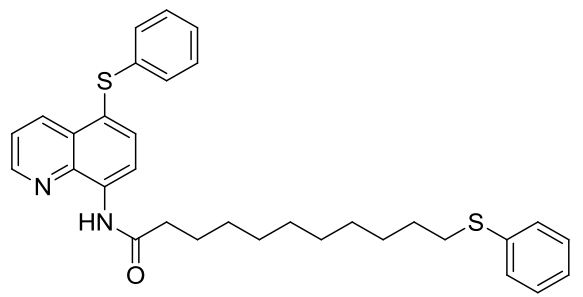

The amide was obtained as yellow solid, $123.7 \mathrm{mg}, 78 \%$ yield. Mp: $80-81{ }^{\circ} \mathrm{C} .{ }^{1} \mathrm{H}$ NMR $\left(400 \mathrm{MHz}, \mathrm{CDCl}_{3}\right): \delta$ $9.83(\mathrm{~s}, 1 \mathrm{H}), 8.71(\mathrm{~d}, J=7.9 \mathrm{~Hz}, 2 \mathrm{H}), 8.59(\mathrm{~d}, J=8.5$ $\mathrm{Hz}, 1 \mathrm{H}), 7.78(\mathrm{~d}, J=8.1 \mathrm{~Hz}, 1 \mathrm{H}), 7.37(\mathrm{dd}, J=8.3,4.0$ $\mathrm{Hz}, 1 \mathrm{H}), 7.19(\mathrm{dd}, J=15.6,8.3 \mathrm{~Hz}, 4 \mathrm{H}), 7.10-7.00(\mathrm{~m}$, $6 \mathrm{H}), 2.82(\mathrm{t}, J=7.3 \mathrm{~Hz}, 2 \mathrm{H}), 2.49(\mathrm{t}, J=7.5 \mathrm{~Hz}, 2 \mathrm{H})$, $1.76-1.70(\mathrm{~m}, 2 \mathrm{H}), 1.62-1.48(\mathrm{~m}, 2 \mathrm{H}), 1.32-1.13(\mathrm{~m}, 12 \mathrm{H}) .{ }^{13} \mathrm{C} \mathrm{NMR}\left(101 \mathrm{MHz}, \mathrm{CDCl}_{3}\right): \delta$ $172.0,148.3$, 139.1, 137.8, 137.0, 136.2, 136.0, 134.9, 129.3, 129.0, 128.8, 128.8, 127.4, 125.7, 125.6, 122.6, 122.3, 116.2, 38.2, 33.5, 29.7, 29.4, 29.3, 29.3, 29.2, 29.1, 28.8, 25.6. HRMS m/z (EI) calcd for $\left[\mathrm{C}_{32} \mathrm{H}_{36} \mathrm{~N}_{2} \mathrm{OS}_{2}\right]: 528.2269$, found: 528.2273.

$N$-(5-(phenylselanyl)quinolin-8-yl)pivalamide (5a)

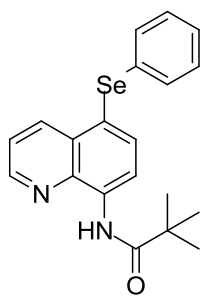

The amide was obtained as white solid, $93.3 \mathrm{mg}, 81 \%$ yield. Mp: $154-155{ }^{\circ} \mathrm{C}$. ${ }^{1} \mathrm{H}$ NMR (400 MHz, $\left.\mathrm{CDCl}_{3}\right): \delta 10.27(\mathrm{~s}, 1 \mathrm{H}), 8.74-8.63(\mathrm{~m}, 2 \mathrm{H}), 8.55(\mathrm{~d}, J=$ $8.4 \mathrm{~Hz}, 1 \mathrm{H}), 7.89$ (d, $J=8.0 \mathrm{~Hz}, 1 \mathrm{H}), 7.33(\mathrm{dd}, J=8.4,4.1 \mathrm{~Hz}, 1 \mathrm{H}), 7.12-6.98$ $(\mathrm{m}, 5 \mathrm{H}), 1.34(\mathrm{~s}, 9 \mathrm{H}) .{ }^{13} \mathrm{C}$ NMR $\left(101 \mathrm{MHz}, \mathrm{CDCl}_{3}\right): \delta 177.3,148.3,139.4$, 137.4, 137.0, 136.2, 132.6, 130.0, 129.7, 129.2, 126.3, 122.4, 120.3, 116.2, 40.4, 27.7. HRMS m/z (EI) calcd for $\left[\mathrm{C}_{20} \mathrm{H}_{20} \mathrm{~N}_{2} \mathrm{OSe}\right]$ : 384.0741 , found: 384.0744 .

$N$-(5-((4-chlorophenyl)selanyl)quinolin-8-yl)pivalamide (5b)

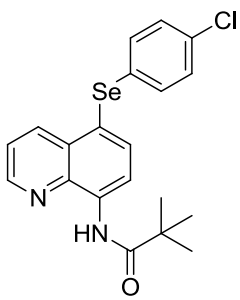

The amide was obtained as yellow solid, $93.8 \mathrm{mg}, 75 \%$ yield. Mp: 155-156 ${ }^{\circ} \mathrm{C} .{ }^{1} \mathrm{H}$ NMR $\left(400 \mathrm{MHz}, \mathrm{CDCl}_{3}\right) \delta 10.38(\mathrm{~s}, 1 \mathrm{H}), 8.84-8.73(\mathrm{~m}, 2 \mathrm{H}), 8.61(\mathrm{~d}$, $J=8.5 \mathrm{~Hz}, 1 \mathrm{H}), 7.99(\mathrm{~d}, J=7.4 \mathrm{~Hz}, 1 \mathrm{H}), 7.46(\mathrm{dd}, J=7.6,3.3 \mathrm{~Hz}, 1 \mathrm{H}), 7.10$ $(\mathrm{d}, J=0.9 \mathrm{~Hz}, 4 \mathrm{H}), 1.44(\mathrm{~s}, 9 \mathrm{H}) .{ }^{13} \mathrm{C} \mathrm{NMR}\left(101 \mathrm{MHz}, \mathrm{CDCl}_{3}\right): \delta 177.4$, $148.4,139.4,137.7,136.9,136.5,132.4,131.1,130.9,129.6,129.3,122.5$, 119.7, 116.2, 40.4, 27.7. HRMS m/z (EI) calcd for $\left[\mathrm{C}_{20} \mathrm{H}_{19} \mathrm{ClN}_{2} \mathrm{OSe}\right]$ : 418.0351, found: 418.0355 . 


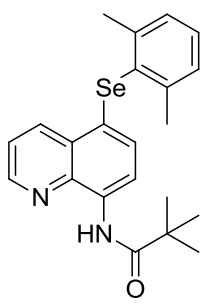

The amide was obtained as yellow oil, $96.4 \mathrm{mg}, 78 \%$ yield. ${ }^{1} \mathrm{H}$ NMR (400 MHz, $\left.\mathrm{CDCl}_{3}\right) \delta 10.21(\mathrm{~s}, 1 \mathrm{H}), 8.83(\mathrm{~d}, J=4.1 \mathrm{~Hz}, 1 \mathrm{H}), 8.55(\mathrm{~d}, J=8.2 \mathrm{~Hz}, 2 \mathrm{H}), 7.50$ $(\mathrm{dd}, J=8.5,4.2 \mathrm{~Hz}, 1 \mathrm{H}), 7.25-7.19(\mathrm{~m}, 1 \mathrm{H}), 7.16(\mathrm{~d}, J=7.2 \mathrm{~Hz}, 2 \mathrm{H}), 7.07$ (d, $J=8.2 \mathrm{~Hz}, 1 \mathrm{H}), 2.44(\mathrm{~s}, 6 \mathrm{H}), 1.40(\mathrm{~s}, 9 \mathrm{H}),{ }^{13} \mathrm{C} \mathrm{NMR}\left(101 \mathrm{MHz}, \mathrm{CDCl}_{3}\right): \delta$ $177.0,148.2$, 143.5, 139.3, 134.8, 133.4, 130.3, 129.2, 128.6, 128.2, 127.8, 124.0, 121.5, 116.7, 40.29, 27.7, 24.3. HRMS m/z (EI) calcd for $\left[\mathrm{C}_{22} \mathrm{H}_{24} \mathrm{~N}_{2} \mathrm{OSe}\right]$ 412.1054, found: 412.1059 .

$N$-(5-(mesitylselanyl)quinolin-8-yl)pivalamide (5d)

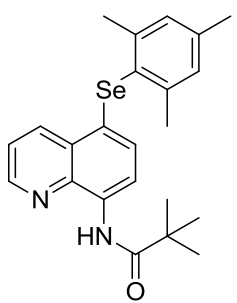

The amide was obtained as yellow solid, $96.9 \mathrm{mg}, 76 \%$ yield. Mp: $144-146{ }^{\circ} \mathrm{C}$. ${ }^{1} \mathrm{H}$ NMR (400 MHz, $\left.\mathrm{CDCl}_{3}\right) \delta 10.20(\mathrm{~s}, 1 \mathrm{H}), 8.82(\mathrm{~d}, J=4.0 \mathrm{~Hz}, 1 \mathrm{H}), 8.54(\mathrm{t}$, $J=7.5 \mathrm{~Hz}, 2 \mathrm{H}), 7.50(\mathrm{dd}, J=8.4,4.1 \mathrm{~Hz}, 1 \mathrm{H}), 7.08-6.97(\mathrm{~m}, 3 \mathrm{H}), 2.40$ (s, $6 \mathrm{H}), 2.32(\mathrm{~s}, 3 \mathrm{H}), 1.40(\mathrm{~s}, 9 \mathrm{H}) .{ }^{13} \mathrm{C} \mathrm{NMR}\left(101 \mathrm{MHz}, \mathrm{CDCl}_{3}\right): \delta 177.0,148.2$, $143.4,139.3,139.2$, 134.8, 133.2, 129.1, 128.2, 127.7, 126.6, 124.4, 121.4, 116.8, 40.3, 27.7, 24.2, 21.0. HRMS m/z (EI) calcd for $\left[\mathrm{C}_{23} \mathrm{H}_{26} \mathrm{~N}_{2} \mathrm{OSe}\right]$ : 426.1210, found: 426.1204 .

$N$-(5-((4-chlorophenyl)selanyl)quinolin-8-yl)-2,2-dimethylbutanamide (5e)

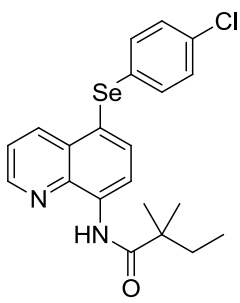

The amide was obtained as yellow solid, 93,3 mg, 72\% yield. Mp: 145-146 ${ }^{\circ} \mathrm{C} .{ }^{1} \mathrm{H}$ NMR $\left(400 \mathrm{MHz}, \mathrm{CDCl}_{3}\right) \delta 10.34(\mathrm{~s}, 1 \mathrm{H}), 8.79(\mathrm{t}, J=6.6 \mathrm{~Hz}, 2 \mathrm{H})$, $8.60(\mathrm{~d}, J=8.5 \mathrm{~Hz}, 1 \mathrm{H}), 7.99(\mathrm{~d}, J=8.1 \mathrm{~Hz}, 1 \mathrm{H}), 7.45(\mathrm{dd}, J=8.5,4.2 \mathrm{~Hz}$, 1H), $7.09(\mathrm{~s}, 4 \mathrm{H}), 1.78(\mathrm{dd}, J=14.8,7.3 \mathrm{~Hz}, 2 \mathrm{H}), 1.40(\mathrm{~s}, 6 \mathrm{H}), 0.97(\mathrm{t}, J=$ $7.4 \mathrm{~Hz}, 3 \mathrm{H}) .{ }^{13} \mathrm{C}$ NMR $\left(101 \mathrm{MHz}, \mathrm{CDCl}_{3}\right): \delta 176.8,148.4,139.4,137.7$, $136.8,136.4,132.4,131.1,130.9,129.6,129.3,122.5,119.7,116.2,44.1$,

34.0, 25.0, 9.3. HRMS m/z (EI) calcd for $\left[\mathrm{C}_{21} \mathrm{H}_{21} \mathrm{ClN}_{2} \mathrm{OSe}\right]$ : 432.0508 , found: 432.0501 .

$N$-(5-((4-chlorophenyl)selanyl)quinolin-8-yl)acetamide (5f)

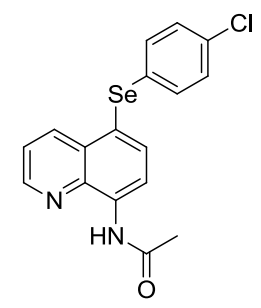

The amide was obtained as yellow solid, $76.7 \mathrm{mg}, 68 \%$ yield. Mp: 146-148 ${ }^{\circ} \mathrm{C} .{ }^{1} \mathrm{H}$ NMR $\left(400 \mathrm{MHz}, \mathrm{CDCl}_{3}\right) \delta 9.89(\mathrm{~s}, 1 \mathrm{H}), 8.80(\mathrm{~d}, J=3.3 \mathrm{~Hz}, 1 \mathrm{H}), 8.73$ $(\mathrm{d}, J=8.0 \mathrm{~Hz}, 1 \mathrm{H}), 8.63(\mathrm{~d}, J=8.4 \mathrm{~Hz}, 1 \mathrm{H}), 7.98(\mathrm{~d}, J=8.0 \mathrm{~Hz}, 1 \mathrm{H}), 7.48$ $(\mathrm{dd}, J=8.5,4.2 \mathrm{~Hz}, 1 \mathrm{H}), 7.12$ (s, 4H), 2.37 (s, 3H). ${ }^{13} \mathrm{C}$ NMR (101 MHz, $\left.\mathrm{CDCl}_{3}\right): \delta 168.9,148.4,138.9,137.5,136.9,136.3,132.6,131.4,130.7,129.6$, 129.4, 122.6, 120.2, 116.5, 25.1. HRMS m/z (EI) calcd for $\left[\mathrm{C}_{17} \mathrm{H}_{13} \mathrm{ClN}_{2} \mathrm{OSe}\right]$ : 375.9882, found: 375.9875 .

$N$-(5-((4-chlorophenyl)selanyl)quinolin-8-yl)cyclopentanecarboxamide (5g)

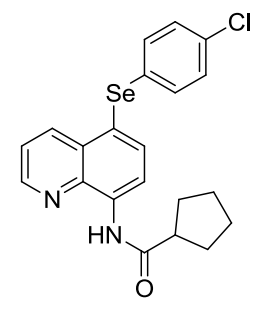

The amide was obtained as yellow solid, $96.7 \mathrm{mg}, 75 \%$ yield. Mp: 154-155 ${ }^{\circ} \mathrm{C} .{ }^{1} \mathrm{H}$ NMR $\left(400 \mathrm{MHz}, \mathrm{CDCl}_{3}\right) \delta 9.96(\mathrm{~s}, 1 \mathrm{H}), 8.85-8.71(\mathrm{~m}, 2 \mathrm{H}), 8.59(\mathrm{~d}$, 
$J=8.5 \mathrm{~Hz}, 1 \mathrm{H}), 7.97(\mathrm{~d}, J=8.1 \mathrm{~Hz}, 1 \mathrm{H}), 7.44(\mathrm{dd}, J=8.5,4.2 \mathrm{~Hz}, 1 \mathrm{H}), 7.09$ (s, 4H), $2.99-$ $2.91(\mathrm{~m}, 1 \mathrm{H}), 2.11-1.92(\mathrm{~m}, 4 \mathrm{H}), 1.89-1.77(\mathrm{~m}, 2 \mathrm{H}), 1.72-1.65(\mathrm{~m}, 2 \mathrm{H}) .{ }^{13} \mathrm{C}$ NMR $(101$ $\left.\mathrm{MHz}, \mathrm{CDCl}_{3}\right): \delta 175.2,148.3,139.0,137.6,136.8,136.4,132.4,131.1,130.9,129.5,129.3$, 122.5, 119.7, 116.3, 47.3, 30.5, 25.9. HRMS m/z (EI) calcd for $\left[\mathrm{C}_{21} \mathrm{H}_{19} \mathrm{ClN}_{2} \mathrm{OSe}\right]: 430.0351$, found: 430.0345 .

5-(phenylthio)quinolin-8-amine (6)<smiles>Nc1ccc(Sc2ccccc2)c2cccnc12</smiles>

To a solution of $N$-(5-(phenylthio)quinolin-8-yl)pivalamide 3a $(0.2 \mathrm{mmol}$, $67.2 \mathrm{mg}$ ) in EtOH $(1.0 \mathrm{~mL})$, was added $\mathrm{NaOH}(20 \mathrm{mmol}, 80 \mathrm{mg})$. The mixture was heated in $100{ }^{\circ} \mathrm{C}$ for $12 \mathrm{~h}$. Then, the mixture was concentrated under reduced pressure. The residue was purified by flash chromatography (silica gel, petroleum ether / ethyl acetate $=5: 1, \mathrm{v} / \mathrm{v}$ ) to afford 5-(phenylthio)quinolin-8-amine. ${ }^{\text {la }}$ The amide was obtained as brown thick oil, $42.8 \mathrm{mg}, 85 \%$ yield. ${ }^{1} \mathrm{H} \mathrm{NMR}\left(400 \mathrm{MHz}, \mathrm{CDCl}_{3}\right) \delta 8.76(\mathrm{~d}, J=3.0 \mathrm{~Hz}, 1 \mathrm{H}), 8.59(\mathrm{~d}, J=8.5 \mathrm{~Hz}$, $1 \mathrm{H}), 7.74(\mathrm{~d}, J=7.8 \mathrm{~Hz}, 1 \mathrm{H}), 7.39(\mathrm{dd}, J=8.4,4.0 \mathrm{~Hz}, 1 \mathrm{H}), 7.14(\mathrm{t}, J=7.5 \mathrm{~Hz}, 2 \mathrm{H}), 7.08-$ $6.88(\mathrm{~m}, 4 \mathrm{H}), 5.28(\mathrm{~s}, 2 \mathrm{H}) .{ }^{13} \mathrm{C} \mathrm{NMR}\left(101 \mathrm{MHz}, \mathrm{CDCl}_{3}\right): \delta 147.5,146.1,139.5,138.9,137.6$, 134.6, 130.6, 128.8, 126.0, 124.9, 122.4, 114.0, 109.4. HRMS m/z (EI) calcd for $\left[\mathrm{C}_{15} \mathrm{H}_{12} \mathrm{~N}_{2} \mathrm{~S}\right]$ : 252.0721, found: 252.0723 .

$N$-(5-(phenylsulfonyl)quinolin-8-yl)pivalamide (7)

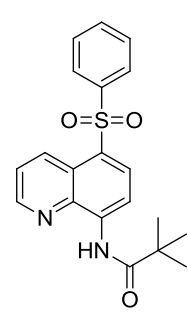

To a solution of $N$-(5-(phenylthio)quinolin-8-yl)pivalamide $3 \mathbf{a}(0.2 \mathrm{mmol}, 67.2 \mathrm{mg})$ in $\mathrm{CH}_{2} \mathrm{Cl}_{2}(3.0 \mathrm{~mL})$ at $0{ }^{\circ} \mathrm{C}$, was added $m$-CPBA $(92 \mathrm{mg}, 0.44 \mathrm{mmol})$. The reaction mixture was stirred vigorously for $1 \mathrm{~h}$, during which the temperature was slowly allowed to raise from $0{ }^{\circ} \mathrm{C}$ to room temperature. Then, aqueous saturated solution of $\mathrm{Na}_{2} \mathrm{SO}_{3}(10 \mathrm{~mL})$ was added. The organic phase was separated, washed with aqueous saturated solution of $\mathrm{NaHCO}_{3}(2 \times 10 \mathrm{~mL})$, dried $\left(\mathrm{Na}_{2} \mathrm{SO}_{4}\right)$ and concentrated under reduced pressure. The residue was purified by flash chromatography (silica gel, petroleum ether / ethyl acetate $=10: 1, \mathrm{v} / \mathrm{v})$ to afford $N$-(5-(phenylsulfonyl)quinolin- 8 -yl)pivalamide. ${ }^{4}$ The amide was obtained as yellow white solid, $60.3 \mathrm{mg}, 82 \%$ yield. Mp: $152-153{ }^{\circ} \mathrm{C} .{ }^{1} \mathrm{H} \mathrm{NMR}\left(400 \mathrm{MHz}, \mathrm{CDCl}_{3}\right) \delta 10.49(\mathrm{~s}$, $1 \mathrm{H}), 9.02(\mathrm{~d}, J=8.7 \mathrm{~Hz}, 1 \mathrm{H}), 8.89(\mathrm{~d}, J=8.4 \mathrm{~Hz}, 1 \mathrm{H}), 8.83(\mathrm{~d}, J=4.0 \mathrm{~Hz}, 1 \mathrm{H}), 8.51(\mathrm{~d}, J=8.4 \mathrm{~Hz}$, $1 \mathrm{H}), 7.91(\mathrm{~d}, J=7.5 \mathrm{~Hz}, 2 \mathrm{H}), 7.62-7.40(\mathrm{~m}, 4 \mathrm{H}), 1.40(\mathrm{~s}, 9 \mathrm{H}) .{ }^{13} \mathrm{C} \mathrm{NMR}\left(101 \mathrm{MHz}, \mathrm{CDCl}_{3}\right): \delta 177.7$, 148.7, 141.9, 140.2, 138.4, 133.2, 133.0, 132.3, 129.2, 128.3, 127.0, 124.2, 123.2, 113.8, 40.5, 27.5. HRMS m/z (EI) calcd for $\left[\mathrm{C}_{20} \mathrm{H}_{20} \mathrm{~N}_{2} \mathrm{O}_{3} \mathrm{~S}\right]: 368.1195$, found: 368.1199 .

2,2-dimethyl-3-(phenylthio)- $N$-(5-(phenylthio)quinolin-8-yl)propanamide (8)

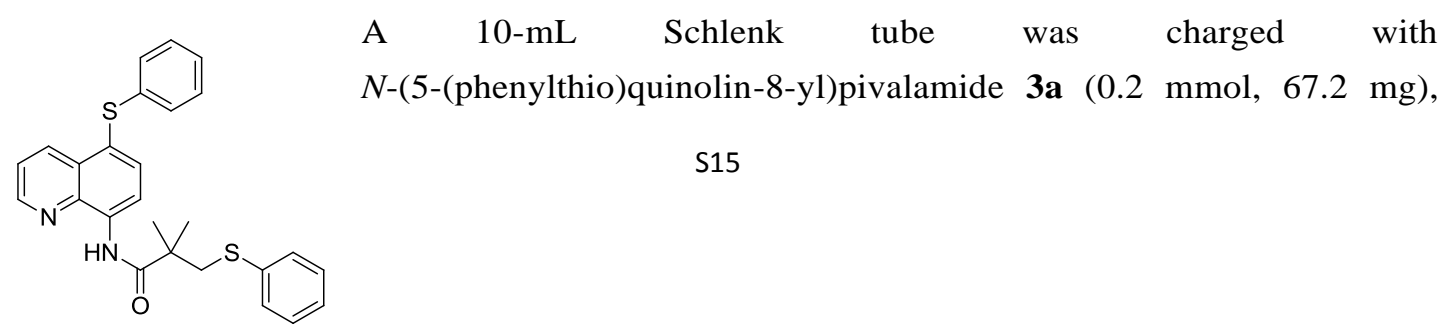


diphenyl disulfide $2 \mathrm{a}(87.3 \mathrm{mg}, 0.4 \mathrm{mmol}), \mathrm{NiBr}_{2}(4.4 \mathrm{mg}, 0.02 \mathrm{mmol})$, MesCOOH (6.6 mg, $0.04 \mathrm{mmol}), \mathrm{Na}_{2} \mathrm{CO}_{3}(42.4 \mathrm{mg}, 0.4 \mathrm{mmol})$ and DMF $(0.5 \mathrm{~mL})$. The vial was evacuated and filled with $\mathrm{N}_{2}$, and stirred at $160{ }^{\circ} \mathrm{C}$ for $24 \mathrm{~h}$. The mixture was then cooled to room temperature, diluted with $\mathrm{CH}_{2} \mathrm{Cl}_{2}(2 \mathrm{~mL})$, filtered through a celite pad, and concentrated in vacuo. The residue was purified by flash column chromatography on silica gel, eluting with EtOAc/Hexane $(1: 100 \sim 1: 60, \quad \mathrm{v} / \mathrm{v}), \quad$ to afford the desired thiolated product 2,2-dimethyl-3-(phenylthio)- $N$-(5-(phenylthio)quinolin-8-yl)propanamide. ${ }^{\text {la }}$ The amide was obtained as yellow solid, $37.3 \mathrm{mg}, 42 \%$ yield. Mp: $100-101{ }^{\circ} \mathrm{C} .{ }^{1} \mathrm{H}$ NMR $\left(400 \mathrm{MHz}, \mathrm{CDCl}_{3}\right) \delta$ $10.45(\mathrm{~s}, 1 \mathrm{H}), 8.81(\mathrm{dd}, J=4.1,1.3 \mathrm{~Hz}, 1 \mathrm{H}), 8.73(\mathrm{~d}, J=8.1 \mathrm{~Hz}, 1 \mathrm{H}), 8.67(\mathrm{dd}, J=8.5,1.3 \mathrm{~Hz}$, $1 \mathrm{H}), 7.85(\mathrm{~d}, J=8.1 \mathrm{~Hz}, 1 \mathrm{H}), 7.47(\mathrm{dd}, J=8.5,4.2 \mathrm{~Hz}, 1 \mathrm{H}), 7.38(\mathrm{~d}, J=7.3 \mathrm{~Hz}, 2 \mathrm{H}), 7.21-$ $7.03(\mathrm{~m}, 8 \mathrm{H}), 3.37(\mathrm{~s}, 2 \mathrm{H}), 1.55(\mathrm{~s}, 6 \mathrm{H}) .{ }^{13} \mathrm{C} \mathrm{NMR}\left(101 \mathrm{MHz}, \mathrm{CDCl}_{3}\right): \delta 174.8,148.4,139.5$, 137.9, 136.8, 136.2, 135.8, 134.9, 130.0, 129.3, 129.0, 128.7, 127.4, 126.1, 125.7, 122.7, 122.4, 116.3, 45.4, 45.4, 25.2. One signal missing. HRMS m/z (EI) calcd for $\left[\mathrm{C}_{26} \mathrm{H}_{24} \mathrm{~N}_{2} \mathrm{OS}_{2}\right]: 444.1330$, found: 444.1334 .

\section{Unsuccessful examples}

(1) Aryl-substituted aminoquinolines with low regioselectively:<smiles>O=C(Nc1cccc2cccnc12)c1ccccc1</smiles><smiles>Cc1ccc(C(=O)Nc2cccc3cccnc23)cc1</smiles><smiles>COc1ccc(C(=O)Nc2cccc3cccnc23)cc1</smiles>

(2) Ditellurides with low efficiency:<smiles>Cc1cc(C)c([Te][Te]c2c(C)cc(C)cc2C)c(C)c1</smiles><smiles>Cc1cccc(C)c1[Te][Te]c1c(C)cccc1C</smiles><smiles>Clc1ccccc1[Te]c1ccccc1Cl</smiles> 


\section{GC-MS spectrum of adduct 12}

$\mathrm{E}: \backslash$ 个人数据结果 $\backslash$ 朱龙志 $\backslash 20151026-\mathrm{R} 537+\mathrm{BHT} . \mathrm{qgd}$
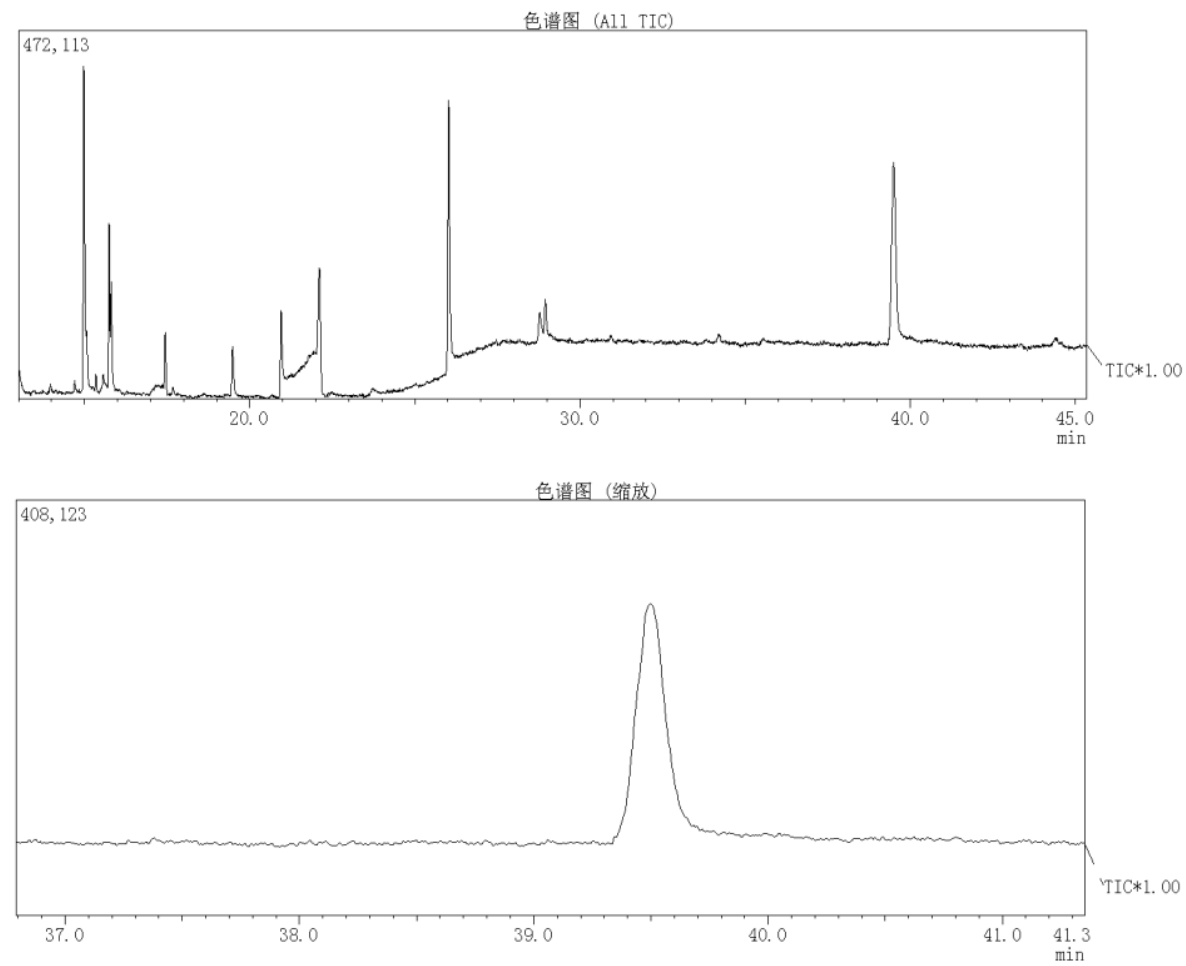

质谱

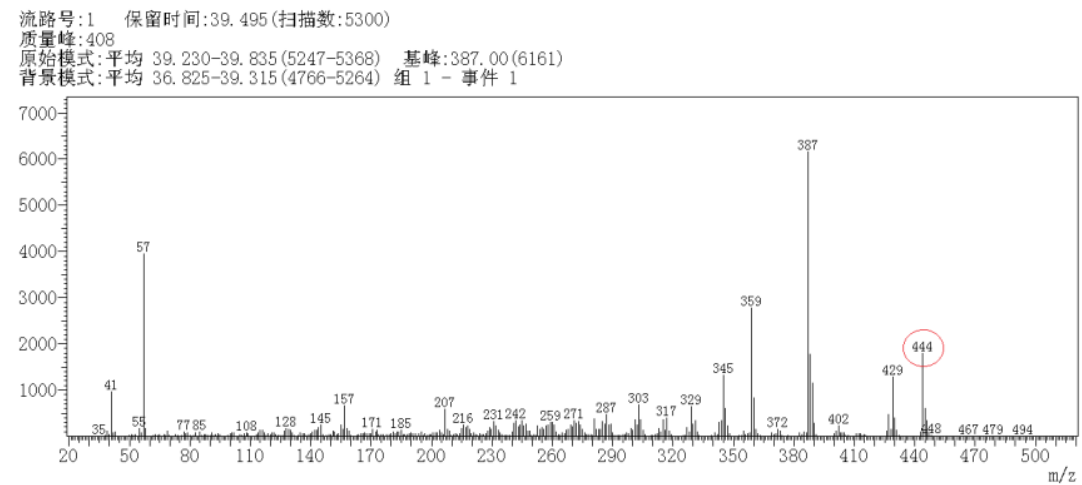




\section{Referrences}

(1) (a) Wang, X.; Qiu, R.; Yan, C.; Reddy, V. P.; Zhu, L.; Xu, X.; Yin, S.-F., Org. Lett. 2015, 17, 1970. (b) Wu, X.; Zhao, Y.; Ge, H., J. Am. Chem. Soc. 2014, 136, 1789. (c) Aihara, Y.; Chatani, N., J. Am. Chem. Soc. 2014, 136, 898.

(2) Kathiravan, S.; Ghosh, S.; Hogarth, G.; Nicholls, I. A., Chem. Commun. 2015, 51, 4834.

(3) Sun, W. W.; Cao, P.; Mei, R. Q.; Li, Y.; Ma, Y. L.; Wu, B., Org. Lett. 2014, 16, 480.

(4) (a) Yao, J.; Yu, M.; Zhang, Y., Adv. Synth. Catal. 2012, 354, 3205. (b) Yu, M.; Xie, Y.; Xie, C.; Zhang, Y., Org. Lett. 2012, 14, 2164. 


\section{X-ray information of Complex 3a (CCDC 1419167 )}

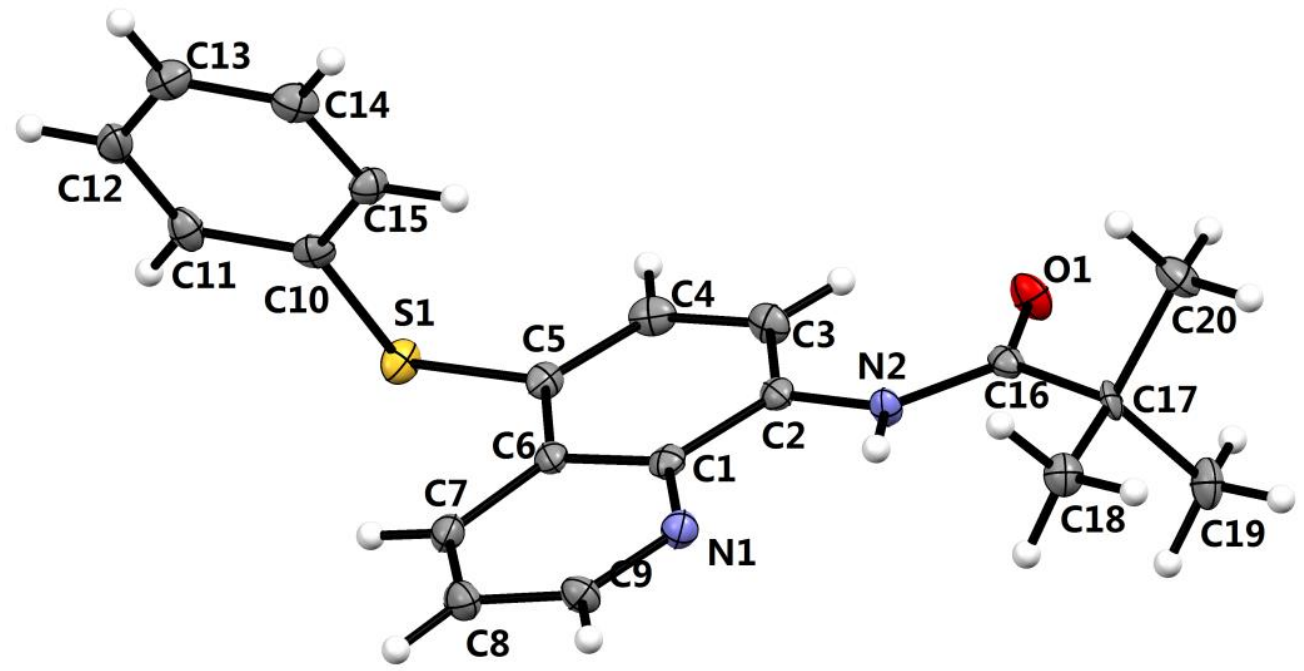

Table 1. Crystal data and structure refinement for mo_20150423D_0m.

Identification code

Empirical formula

Formula weight

Temperature

Wavelength

Crystal system, space group

Unit cell dimensions

Volume

Z, Calculated density

Absorption coefficient

$\mathrm{F}(000)$

Crystal size

Theta range for data collection

Limiting indices

Reflections collected / unique

Completeness to theta $=25.50$

Absorption correction

Max. and min. transmission

Refinement method

$$
\begin{aligned}
& \text { mo_20150423d_0m } \\
& \text { C20 H20 N2 O S } \\
& 336.44 \\
& 100(2) \mathrm{K} \\
& 0.71073 \text { A }
\end{aligned}
$$

Monoclinic, P 21/n

$\mathrm{a}=10.5944(18)$ A alpha $=90 \mathrm{deg}$.

$\mathrm{b}=5.6765(10) \mathrm{A} \quad$ beta $=94.681(3) \mathrm{deg}$.

$\mathrm{c}=27.958(5) \mathrm{A} \quad$ gamma $=90 \mathrm{deg}$.

$1675.8(5) \mathrm{A}^{\wedge} 3$

$4,1.334 \mathrm{Mg} / \mathrm{m}^{\wedge} 3$

$0.202 \mathrm{~mm}^{\wedge}-1$

712

$0.25 \times 0.22 \times 0.18 \mathrm{~mm}$

1.46 to $25.50 \mathrm{deg}$.

$-12<=\mathrm{h}<=10,-6<=\mathrm{k}<=6,-33<=\mathrm{l}<=33$

$12220 / 3048[\mathrm{R}(\mathrm{int})=0.0341]$

$97.7 \%$

Semi-empirical from equivalents

0.9645 and 0.9512

Full-matrix least-squares on $\mathrm{F}^{\wedge} 2$ 
Data / restraints / parameters

Goodness-of-fit on $\mathrm{F}^{\wedge} 2$

Final $\mathrm{R}$ indices $[\mathrm{I}>2 \operatorname{sigma}(\mathrm{I})]$

$\mathrm{R}$ indices (all data)

Largest diff. peak and hole
3048 / 0 / 220

1.179

$\mathrm{R} 1=0.0913, \mathrm{wR} 2=0.2157$

$\mathrm{R} 1=0.0953, \mathrm{wR} 2=0.2174$

0.658 and -0.457 e. $A^{\wedge}-3$

Table 2. Atomic coordinates ( x 10^4) and equivalent isotropic displacement parameters $\left(\mathrm{A}^{\wedge} 2 \times 10^{\wedge} 3\right)$ for mo_20150423D_0m.

$\mathrm{U}(\mathrm{eq})$ is defined as one third of the trace of the orthogonalized Uij tensor.

\begin{tabular}{|c|c|c|c|c|}
\hline & $\mathrm{x}$ & $\mathrm{y}$ & $\mathrm{z}$ & $\mathrm{U}(\mathrm{eq})$ \\
\hline$S(1)$ & $2436(1)$ & $3969(2)$ & 137(1) & $18(1)$ \\
\hline $\mathrm{O}(1)$ & $5682(3)$ & $8730(7)$ & $2155(1)$ & $21(1)$ \\
\hline $\mathrm{N}(2)$ & $3845(4)$ & 10272(8) & $1819(1)$ & $14(1)$ \\
\hline $\mathrm{C}(1)$ & 2391(5) & $9134(9)$ & $1166(2)$ & $15(1)$ \\
\hline$C(2)$ & $3570(4)$ & $8701(9)$ & $1438(2)$ & $14(1)$ \\
\hline $\mathrm{C}(3)$ & $4324(5)$ & $6852(9)$ & $1316(2)$ & $17(1)$ \\
\hline$C(4)$ & $3946(5)$ & $5433(9)$ & $916(2)$ & $18(1)$ \\
\hline$C(5)$ & $2811(5)$ & $5774(9)$ & $645(2)$ & $14(1)$ \\
\hline$C(6)$ & $1990(4)$ & $7638(9)$ & $774(2)$ & $14(1)$ \\
\hline$C(7)$ & $775(5)$ & $8078(9)$ & $534(2)$ & $17(1)$ \\
\hline $\mathrm{C}(8)$ & $80(5)$ & $9928(10)$ & $677(2)$ & $20(1)$ \\
\hline $\mathrm{C}(9)$ & $581(5)$ & $11358(9)$ & $1060(2)$ & $17(1)$ \\
\hline $\mathrm{N}(1)$ & 1681(4) & $10977(8)$ & $1300(1)$ & $17(1)$ \\
\hline$C(10)$ & $2790(4)$ & $5861(9)$ & $-345(2)$ & $16(1)$ \\
\hline$C(11)$ & $2420(5)$ & $5084(9)$ & $-811(2)$ & $18(1)$ \\
\hline$C(12)$ & $2683(5)$ & $6456(11)$ & $-1200(2)$ & $22(1)$ \\
\hline$C(13)$ & $3302(5)$ & $8615(10)$ & $-1130(2)$ & $19(1)$ \\
\hline$C(14)$ & $3657(5)$ & $9368(10)$ & $-670(2)$ & $21(1)$ \\
\hline$C(15)$ & $3427(5)$ & $8009(9)$ & $-277(2)$ & $16(1)$ \\
\hline$C(16)$ & $4844(4)$ & 10208(9) & $2160(2)$ & $14(1)$ \\
\hline$C(17)$ & $4854(4)$ & $12034(9)$ & $2565(2)$ & $13(1)$ \\
\hline$C(18)$ & $3788(5)$ & $13854(10)$ & $2504(2)$ & $19(1)$ \\
\hline
\end{tabular}




\begin{tabular}{lllll}
$\mathrm{C}(20)$ & $6146(5)$ & $13282(10)$ & $2590(2)$ & $22(1)$ \\
$\mathrm{C}(19)$ & $4735(5)$ & $10657(10)$ & $3033(2)$ & $21(1)$ \\
\hline
\end{tabular}

Table 3. Bond lengths [A] and angles [deg] for mo_20150423D_0m.

\begin{tabular}{|c|c|}
\hline$S(1)-C(5)$ & $1.770(5)$ \\
\hline$S(1)-C(10)$ & $1.787(5)$ \\
\hline $\mathrm{O}(1)-\mathrm{C}(16)$ & $1.223(6)$ \\
\hline$N(2)-C(16)$ & $1.366(6)$ \\
\hline $\mathrm{N}(2)-\mathrm{C}(2)$ & $1.402(6)$ \\
\hline $\mathrm{N}(2)-\mathrm{H}(2)$ & 0.8600 \\
\hline $\mathrm{C}(1)-\mathrm{N}(1)$ & $1.359(7)$ \\
\hline$C(1)-C(6)$ & $1.423(7)$ \\
\hline$C(1)-C(2)$ & $1.430(7)$ \\
\hline$C(2)-C(3)$ & $1.378(7)$ \\
\hline$C(3)-C(4)$ & $1.409(7)$ \\
\hline $\mathrm{C}(3)-\mathrm{H}(3)$ & 0.9300 \\
\hline$C(4)-C(5)$ & $1.381(7)$ \\
\hline $\mathrm{C}(4)-\mathrm{H}(4)$ & 0.9300 \\
\hline$C(5)-C(6)$ & $1.434(7)$ \\
\hline$C(6)-C(7)$ & $1.424(7)$ \\
\hline$C(7)-C(8)$ & $1.361(7)$ \\
\hline $\mathrm{C}(7)-\mathrm{H}(7)$ & 0.9300 \\
\hline $\mathrm{C}(8)-\mathrm{C}(9)$ & $1.414(7)$ \\
\hline $\mathrm{C}(8)-\mathrm{H}(8)$ & 0.9300 \\
\hline$C(9)-N(1)$ & $1.313(6)$ \\
\hline $\mathrm{C}(9)-\mathrm{H}(9)$ & 0.9300 \\
\hline $\mathrm{C}(10)-\mathrm{C}(15)$ & $1.399(7)$ \\
\hline $\mathrm{C}(10)-\mathrm{C}(11)$ & $1.401(7)$ \\
\hline $\mathrm{C}(11)-\mathrm{C}(12)$ & $1.384(8)$ \\
\hline $\mathrm{C}(11)-\mathrm{H}(11)$ & 0.9300 \\
\hline $\mathrm{C}(12)-\mathrm{C}(13)$ & $1.396(8)$ \\
\hline $\mathrm{C}(12)-\mathrm{H}(12)$ & 0.9300 \\
\hline$C(13)-C(14)$ & $1.380(7)$ \\
\hline
\end{tabular}




\begin{tabular}{|c|c|}
\hline $\mathrm{C}(13)-\mathrm{H}(13)$ & 0.9300 \\
\hline$C(14)-C(15)$ & $1.378(7)$ \\
\hline $\mathrm{C}(14)-\mathrm{H}(14)$ & 0.9300 \\
\hline $\mathrm{C}(15)-\mathrm{H}(15)$ & 0.9300 \\
\hline$C(16)-C(17)$ & $1.535(7)$ \\
\hline $\mathrm{C}(17)-\mathrm{C}(18)$ & $1.530(7)$ \\
\hline $\mathrm{C}(17)-\mathrm{C}(19)$ & $1.537(7)$ \\
\hline $\mathrm{C}(17)-\mathrm{C}(20)$ & $1.538(7)$ \\
\hline $\mathrm{C}(18)-\mathrm{H}(18 \mathrm{~A})$ & 0.9600 \\
\hline $\mathrm{C}(18)-\mathrm{H}(18 \mathrm{~B})$ & 0.9600 \\
\hline $\mathrm{C}(18)-\mathrm{H}(18 \mathrm{C})$ & 0.9600 \\
\hline $\mathrm{C}(20)-\mathrm{H}(20 \mathrm{~A})$ & 0.9600 \\
\hline $\mathrm{C}(20)-\mathrm{H}(20 \mathrm{~B})$ & 0.9600 \\
\hline $\mathrm{C}(20)-\mathrm{H}(20 \mathrm{C})$ & 0.9600 \\
\hline $\mathrm{C}(19)-\mathrm{H}(19 \mathrm{~A})$ & 0.9600 \\
\hline $\mathrm{C}(19)-\mathrm{H}(19 \mathrm{~B})$ & 0.9600 \\
\hline C(19)-H(19C) & 0.9600 \\
\hline$C(5)-S(1)-C(10)$ & $102.2(2)$ \\
\hline $\mathrm{C}(16)-\mathrm{N}(2)-\mathrm{C}(2)$ & $127.9(4)$ \\
\hline $\mathrm{C}(16)-\mathrm{N}(2)-\mathrm{H}(2)$ & 116.0 \\
\hline $\mathrm{C}(2)-\mathrm{N}(2)-\mathrm{H}(2)$ & 116.0 \\
\hline$N(1)-C(1)-C(6)$ & $122.3(4)$ \\
\hline $\mathrm{N}(1)-\mathrm{C}(1)-\mathrm{C}(2)$ & $117.7(4)$ \\
\hline$C(6)-C(1)-C(2)$ & $120.0(5)$ \\
\hline $\mathrm{C}(3)-\mathrm{C}(2)-\mathrm{N}(2)$ & $125.7(4)$ \\
\hline$C(3)-C(2)-C(1)$ & $119.9(5)$ \\
\hline$N(2)-C(2)-C(1)$ & $114.4(4)$ \\
\hline$C(2)-C(3)-C(4)$ & $119.9(5)$ \\
\hline $\mathrm{C}(2)-\mathrm{C}(3)-\mathrm{H}(3)$ & 120.0 \\
\hline $\mathrm{C}(4)-\mathrm{C}(3)-\mathrm{H}(3)$ & 120.0 \\
\hline$C(5)-C(4)-C(3)$ & $122.2(5)$ \\
\hline $\mathrm{C}(5)-\mathrm{C}(4)-\mathrm{H}(4)$ & 118.9 \\
\hline $\mathrm{C}(3)-\mathrm{C}(4)-\mathrm{H}(4)$ & 118.9 \\
\hline$C(4)-C(5)-C(6)$ & $119.0(4)$ \\
\hline
\end{tabular}




\begin{tabular}{|c|c|}
\hline$C(4)-C(5)-S(1)$ & $119.1(4)$ \\
\hline$C(6)-C(5)-S(1)$ & $121.8(4)$ \\
\hline$C(1)-C(6)-C(7)$ & $117.3(5)$ \\
\hline$C(1)-C(6)-C(5)$ & $119.0(4)$ \\
\hline$C(7)-C(6)-C(5)$ & $123.7(5)$ \\
\hline$C(8)-C(7)-C(6)$ & $119.2(5)$ \\
\hline $\mathrm{C}(8)-\mathrm{C}(7)-\mathrm{H}(7)$ & 120.4 \\
\hline $\mathrm{C}(6)-\mathrm{C}(7)-\mathrm{H}(7)$ & 120.4 \\
\hline$C(7)-C(8)-C(9)$ & $119.1(5)$ \\
\hline $\mathrm{C}(7)-\mathrm{C}(8)-\mathrm{H}(8)$ & 120.4 \\
\hline $\mathrm{C}(9)-\mathrm{C}(8)-\mathrm{H}(8)$ & 120.4 \\
\hline $\mathrm{N}(1)-\mathrm{C}(9)-\mathrm{C}(8)$ & $123.7(5)$ \\
\hline $\mathrm{N}(1)-\mathrm{C}(9)-\mathrm{H}(9)$ & 118.2 \\
\hline $\mathrm{C}(8)-\mathrm{C}(9)-\mathrm{H}(9)$ & 118.2 \\
\hline $\mathrm{C}(9)-\mathrm{N}(1)-\mathrm{C}(1)$ & $118.3(4)$ \\
\hline$C(15)-C(10)-C(11)$ & $119.7(5)$ \\
\hline$C(15)-C(10)-S(1)$ & $123.4(4)$ \\
\hline $\mathrm{C}(11)-\mathrm{C}(10)-\mathrm{S}(1)$ & $116.9(4)$ \\
\hline $\mathrm{C}(12)-\mathrm{C}(11)-\mathrm{C}(10)$ & $119.6(5)$ \\
\hline $\mathrm{C}(12)-\mathrm{C}(11)-\mathrm{H}(11)$ & 120.2 \\
\hline $\mathrm{C}(10)-\mathrm{C}(11)-\mathrm{H}(11)$ & 120.2 \\
\hline $\mathrm{C}(11)-\mathrm{C}(12)-\mathrm{C}(13)$ & $120.5(5)$ \\
\hline $\mathrm{C}(11)-\mathrm{C}(12)-\mathrm{H}(12)$ & 119.8 \\
\hline $\mathrm{C}(13)-\mathrm{C}(12)-\mathrm{H}(12)$ & 119.8 \\
\hline $\mathrm{C}(14)-\mathrm{C}(13)-\mathrm{C}(12)$ & $119.4(5)$ \\
\hline $\mathrm{C}(14)-\mathrm{C}(13)-\mathrm{H}(13)$ & 120.3 \\
\hline $\mathrm{C}(12)-\mathrm{C}(13)-\mathrm{H}(13)$ & 120.3 \\
\hline$C(15)-C(14)-C(13)$ & $121.1(5)$ \\
\hline $\mathrm{C}(15)-\mathrm{C}(14)-\mathrm{H}(14)$ & 119.4 \\
\hline $\mathrm{C}(13)-\mathrm{C}(14)-\mathrm{H}(14)$ & 119.4 \\
\hline$C(14)-C(15)-C(10)$ & $119.7(5)$ \\
\hline $\mathrm{C}(14)-\mathrm{C}(15)-\mathrm{H}(15)$ & 120.2 \\
\hline $\mathrm{C}(10)-\mathrm{C}(15)-\mathrm{H}(15)$ & 120.2 \\
\hline $\mathrm{O}(1)-\mathrm{C}(16)-\mathrm{N}(2)$ & $122.1(5)$ \\
\hline $\mathrm{O}(1)-\mathrm{C}(16)-\mathrm{C}(17)$ & $120.7(4)$ \\
\hline & S23 \\
\hline
\end{tabular}




\begin{tabular}{|c|c|}
\hline $\mathrm{N}(2)-\mathrm{C}(16)-\mathrm{C}(17)$ & $117.1(4)$ \\
\hline$C(18)-C(17)-C(16)$ & $114.4(4)$ \\
\hline$C(18)-C(17)-C(19)$ & $109.1(4)$ \\
\hline$C(16)-C(17)-C(19)$ & $106.8(4)$ \\
\hline$C(18)-C(17)-C(20)$ & $109.9(4)$ \\
\hline$C(16)-C(17)-C(20)$ & $107.3(4)$ \\
\hline C(19)-C(17)-C(20) & $109.2(4)$ \\
\hline $\mathrm{C}(17)-\mathrm{C}(18)-\mathrm{H}(18 \mathrm{~A})$ & 109.5 \\
\hline $\mathrm{C}(17)-\mathrm{C}(18)-\mathrm{H}(18 \mathrm{~B})$ & 109.5 \\
\hline $\mathrm{H}(18 \mathrm{~A})-\mathrm{C}(18)-\mathrm{H}(18 \mathrm{~B})$ & 109.5 \\
\hline $\mathrm{C}(17)-\mathrm{C}(18)-\mathrm{H}(18 \mathrm{C})$ & 109.5 \\
\hline $\mathrm{H}(18 \mathrm{~A})-\mathrm{C}(18)-\mathrm{H}(18 \mathrm{C})$ & 109.5 \\
\hline $\mathrm{H}(18 \mathrm{~B})-\mathrm{C}(18)-\mathrm{H}(18 \mathrm{C})$ & 109.5 \\
\hline $\mathrm{C}(17)-\mathrm{C}(20)-\mathrm{H}(20 \mathrm{~A})$ & 109.5 \\
\hline $\mathrm{C}(17)-\mathrm{C}(20)-\mathrm{H}(20 \mathrm{~B})$ & 109.5 \\
\hline $\mathrm{H}(20 \mathrm{~A})-\mathrm{C}(20)-\mathrm{H}(20 \mathrm{~B})$ & 109.5 \\
\hline $\mathrm{C}(17)-\mathrm{C}(20)-\mathrm{H}(20 \mathrm{C})$ & 109.5 \\
\hline $\mathrm{H}(20 \mathrm{~A})-\mathrm{C}(20)-\mathrm{H}(20 \mathrm{C})$ & 109.5 \\
\hline $\mathrm{H}(20 \mathrm{~B})-\mathrm{C}(20)-\mathrm{H}(20 \mathrm{C})$ & 109.5 \\
\hline $\mathrm{C}(17)-\mathrm{C}(19)-\mathrm{H}(19 \mathrm{~A})$ & 109.5 \\
\hline $\mathrm{C}(17)-\mathrm{C}(19)-\mathrm{H}(19 \mathrm{~B})$ & 109.5 \\
\hline H(19A)-C(19)-H(19B) & 109.5 \\
\hline $\mathrm{C}(17)-\mathrm{C}(19)-\mathrm{H}(19 \mathrm{C})$ & 109.5 \\
\hline H(19A)-C(19)-H(19C) & 109.5 \\
\hline $\mathrm{H}(19 B)-\mathrm{C}(19)-\mathrm{H}(19 \mathrm{C})$ & 109.5 \\
\hline
\end{tabular}

Symmetry transformations used to generate equivalent atoms: 
Table 4. Anisotropic displacement parameters (A^2 x 10^3) for mo_20150423D_0m.

The anisotropic displacement factor exponent takes the form:

$-2 \mathrm{pi}^{\wedge} 2\left[\mathrm{~h}^{\wedge} 2 \mathrm{a}^{* \wedge} 2 \mathrm{U} 11+\ldots+2 \mathrm{~h} \mathrm{k} \mathrm{a} \mathrm{b}^{*} \mathrm{U} 12\right]$

\begin{tabular}{|c|c|c|c|c|c|c|c|}
\hline & U11 & U22 & U33 & U23 & & U13 & U12 \\
\hline $\mathrm{S}(1)$ & $26(1)$ & 11(1) & $17(1)$ & $-3(1)$ & $3(1)$ & $-3(1)$ & \\
\hline $\mathrm{O}(1)$ & $16(2)$ & $19(2)$ & $27(2)$ & $0(2)$ & $-5(2)$ & $6(2)$ & \\
\hline $\mathrm{N}(2)$ & $12(2)$ & $16(2)$ & $14(2)$ & $-4(2)$ & $-1(2)$ & $4(2)$ & \\
\hline$C(1)$ & $17(2)$ & $14(2)$ & $13(2)$ & $3(2)$ & $3(2)$ & $-2(2)$ & \\
\hline$C(2)$ & $14(2)$ & $15(2)$ & $13(2)$ & $4(2)$ & $1(2)$ & $-1(2)$ & \\
\hline $\mathrm{C}(3)$ & $16(2)$ & $15(3)$ & $21(3)$ & $3(2)$ & $0(2)$ & $4(2)$ & \\
\hline$C(4)$ & $19(3)$ & $13(2)$ & $23(3)$ & $4(2)$ & $5(2)$ & $4(2)$ & \\
\hline$C(5)$ & $19(2)$ & $10(2)$ & $15(2)$ & $-2(2)$ & $3(2)$ & $-4(2)$ & \\
\hline$C(6)$ & $14(2)$ & $15(2)$ & $12(2)$ & $2(2)$ & $1(2)$ & $-2(2)$ & \\
\hline $\mathrm{C}(7)$ & $19(3)$ & $19(3)$ & $12(2)$ & $0(2)$ & $1(2)$ & $-1(2)$ & \\
\hline$C(8)$ & $15(2)$ & $25(3)$ & $18(3)$ & $4(2)$ & $-1(2)$ & $1(2)$ & \\
\hline$C(9)$ & $14(2)$ & $17(3)$ & $20(2)$ & $0(2)$ & $1(2)$ & $2(2)$ & \\
\hline $\mathrm{N}(1)$ & $17(2)$ & $18(2)$ & $15(2)$ & $0(2)$ & $1(2)$ & $2(2)$ & \\
\hline$C(10)$ & $13(2)$ & $15(2)$ & $19(2)$ & $2(2)$ & $4(2)$ & $5(2)$ & \\
\hline $\mathrm{C}(11)$ & $15(2)$ & $16(3)$ & $21(3)$ & $-3(2)$ & $-3(2)$ & $3(2)$ & \\
\hline $\mathrm{C}(12)$ & $15(2)$ & $35(3)$ & $16(2)$ & $-5(2)$ & $-1(2)$ & $4(2)$ & \\
\hline $\mathrm{C}(13)$ & $21(3)$ & $18(3)$ & $20(3)$ & $3(2)$ & $5(2)$ & $4(2)$ & \\
\hline$C(14)$ & $19(3)$ & $18(3)$ & $26(3)$ & $-2(2)$ & $4(2)$ & $0(2)$ & \\
\hline$C(15)$ & $18(2)$ & $15(2)$ & $16(2)$ & $-3(2)$ & $4(2)$ & $0(2)$ & \\
\hline$C(16)$ & $12(2)$ & $14(2)$ & $16(2)$ & $3(2)$ & $1(2)$ & $-2(2)$ & \\
\hline $\mathrm{C}(17)$ & $12(2)$ & $14(2)$ & $12(2)$ & $-3(2)$ & $-6(2)$ & $-4(2)$ & \\
\hline$C(18)$ & $20(3)$ & $18(3)$ & $18(2)$ & $0(2)$ & $0(2)$ & $0(2)$ & \\
\hline$C(20)$ & $15(2)$ & $23(3)$ & $28(3)$ & $-3(2)$ & $-2(2)$ & $-5(2)$ & \\
\hline$C(19)$ & $25(3)$ & $21(3)$ & $16(2)$ & $2(2)$ & $-6(2)$ & $2(2)$ & \\
\hline
\end{tabular}

Table 5. Hydrogen coordinates ( $\left.x 10^{\wedge} 4\right)$ and isotropic displacement parameters $\left(\mathrm{A}^{\wedge} 2 \times 10^{\wedge} 3\right)$ for mo_20150423D_0m. 


\begin{tabular}{|c|c|c|c|c|}
\hline & $\mathrm{x}$ & $\mathrm{y}$ & $\mathrm{z}$ & $\mathrm{U}(\mathrm{eq})$ \\
\hline $\mathrm{H}(2)$ & 3320 & 11415 & 1841 & 17 \\
\hline $\mathrm{H}(3)$ & 5081 & 6542 & 1497 & 21 \\
\hline $\mathrm{H}(4)$ & 4478 & 4227 & 832 & 22 \\
\hline $\mathrm{H}(7)$ & 461 & 7113 & 283 & 20 \\
\hline $\mathrm{H}(8)$ & -714 & 10247 & 524 & 23 \\
\hline $\mathrm{H}(9)$ & 105 & 12640 & 1148 & 21 \\
\hline $\mathrm{H}(11)$ & 1999 & 3655 & -859 & 21 \\
\hline $\mathrm{H}(12)$ & 2446 & 5935 & -1509 & 27 \\
\hline $\mathrm{H}(13)$ & 3474 & 9538 & -1392 & 23 \\
\hline $\mathrm{H}(14)$ & 4057 & 10817 & -623 & 25 \\
\hline $\mathrm{H}(15)$ & 3694 & 8517 & 31 & 19 \\
\hline $\mathrm{H}(18 \mathrm{~A})$ & 3830 & 14660 & 2203 & 28 \\
\hline $\mathrm{H}(18 \mathrm{~B})$ & 3880 & 14972 & 2762 & 28 \\
\hline $\mathrm{H}(18 \mathrm{C})$ & 2985 & 13073 & 2507 & 28 \\
\hline $\mathrm{H}(20 \mathrm{~A})$ & 6810 & 12131 & 2630 & 33 \\
\hline $\mathrm{H}(20 \mathrm{~B})$ & 6211 & 14346 & 2858 & 33 \\
\hline $\mathrm{H}(20 \mathrm{C})$ & 6223 & 14148 & 2299 & 33 \\
\hline $\mathrm{H}(19 \mathrm{~A})$ & 3906 & 9973 & 3028 & 31 \\
\hline $\mathrm{H}(19 \mathrm{~B})$ & 4865 & 11708 & 3301 & 31 \\
\hline $\mathrm{H}(19 \mathrm{C})$ & 5362 & 9432 & 3060 & 31 \\
\hline
\end{tabular}


Table 6. Torsion angles [deg] for mo_20150423D_0m.

\begin{tabular}{|c|c|}
\hline $\mathrm{C}(16)-\mathrm{N}(2)-\mathrm{C}(2)-\mathrm{C}(3)$ & $-4.8(8)$ \\
\hline $\mathrm{C}(16)-\mathrm{N}(2)-\mathrm{C}(2)-\mathrm{C}(1)$ & $174.9(5)$ \\
\hline $\mathrm{N}(1)-\mathrm{C}(1)-\mathrm{C}(2)-\mathrm{C}(3)$ & $180.0(4)$ \\
\hline $\mathrm{C}(6)-\mathrm{C}(1)-\mathrm{C}(2)-\mathrm{C}(3)$ & $0.8(7)$ \\
\hline $\mathrm{N}(1)-\mathrm{C}(1)-\mathrm{C}(2)-\mathrm{N}(2)$ & $0.3(6)$ \\
\hline $\mathrm{C}(6)-\mathrm{C}(1)-\mathrm{C}(2)-\mathrm{N}(2)$ & $-178.9(4)$ \\
\hline $\mathrm{N}(2)-\mathrm{C}(2)-\mathrm{C}(3)-\mathrm{C}(4)$ & $-178.7(5)$ \\
\hline $\mathrm{C}(1)-\mathrm{C}(2)-\mathrm{C}(3)-\mathrm{C}(4)$ & $1.7(7)$ \\
\hline $\mathrm{C}(2)-\mathrm{C}(3)-\mathrm{C}(4)-\mathrm{C}(5)$ & $-2.2(8)$ \\
\hline $\mathrm{C}(3)-\mathrm{C}(4)-\mathrm{C}(5)-\mathrm{C}(6)$ & $0.2(7)$ \\
\hline$C(3)-C(4)-C(5)-S(1)$ & $177.9(4)$ \\
\hline $\mathrm{C}(10)-\mathrm{S}(1)-\mathrm{C}(5)-\mathrm{C}(4)$ & $-101.3(4)$ \\
\hline$C(10)-S(1)-C(5)-C(6)$ & $76.3(4)$ \\
\hline $\mathrm{N}(1)-\mathrm{C}(1)-\mathrm{C}(6)-\mathrm{C}(7)$ & $-2.6(7)$ \\
\hline $\mathrm{C}(2)-\mathrm{C}(1)-\mathrm{C}(6)-\mathrm{C}(7)$ & $176.5(4)$ \\
\hline $\mathrm{N}(1)-\mathrm{C}(1)-\mathrm{C}(6)-\mathrm{C}(5)$ & $178.1(4)$ \\
\hline $\mathrm{C}(2)-\mathrm{C}(1)-\mathrm{C}(6)-\mathrm{C}(5)$ & $-2.7(7)$ \\
\hline$C(4)-C(5)-C(6)-C(1)$ & $2.2(7)$ \\
\hline$S(1)-C(5)-C(6)-C(1)$ & $-175.4(4)$ \\
\hline$C(4)-C(5)-C(6)-C(7)$ & $-177.0(5)$ \\
\hline$S(1)-C(5)-C(6)-C(7)$ & $5.4(7)$ \\
\hline $\mathrm{C}(1)-\mathrm{C}(6)-\mathrm{C}(7)-\mathrm{C}(8)$ & $2.2(7)$ \\
\hline$C(5)-C(6)-C(7)-C(8)$ & $-178.5(5)$ \\
\hline $\mathrm{C}(6)-\mathrm{C}(7)-\mathrm{C}(8)-\mathrm{C}(9)$ & $-0.3(7)$ \\
\hline $\mathrm{C}(7)-\mathrm{C}(8)-\mathrm{C}(9)-\mathrm{N}(1)$ & $-1.7(8)$ \\
\hline $\mathrm{C}(8)-\mathrm{C}(9)-\mathrm{N}(1)-\mathrm{C}(1)$ & $1.4(8)$ \\
\hline $\mathrm{C}(6)-\mathrm{C}(1)-\mathrm{N}(1)-\mathrm{C}(9)$ & $0.8(7)$ \\
\hline $\mathrm{C}(2)-\mathrm{C}(1)-\mathrm{N}(1)-\mathrm{C}(9)$ & $-178.3(5)$ \\
\hline$C(5)-S(1)-C(10)-C(15)$ & $10.3(5)$ \\
\hline$C(5)-S(1)-C(10)-C(11)$ & $-170.9(4)$ \\
\hline$C(15)-C(10)-C(11)-C(12)$ & $-0.2(7)$ \\
\hline$S(1)-C(10)-C(11)-C(12)$ & $-179.1(4)$ \\
\hline$C(10)-C(11)-C(12)-C(13)$ & $-0.7(8)$ \\
\hline
\end{tabular}




$\begin{array}{lr}\mathrm{C}(11)-\mathrm{C}(12)-\mathrm{C}(13)-\mathrm{C}(14) & 0.4(8) \\ \mathrm{C}(12)-\mathrm{C}(13)-\mathrm{C}(14)-\mathrm{C}(15) & 1.0(8) \\ \mathrm{C}(13)-\mathrm{C}(14)-\mathrm{C}(15)-\mathrm{C}(10) & -1.9(8) \\ \mathrm{C}(11)-\mathrm{C}(10)-\mathrm{C}(15)-\mathrm{C}(14) & 1.5(7) \\ \mathrm{S}(1)-\mathrm{C}(10)-\mathrm{C}(15)-\mathrm{C}(14) & -179.7(4) \\ \mathrm{C}(2)-\mathrm{N}(2)-\mathrm{C}(16)-\mathrm{O}(1) & 3.2(8) \\ \mathrm{C}(2)-\mathrm{N}(2)-\mathrm{C}(16)-\mathrm{C}(17) & -174.8(4) \\ \mathrm{O}(1)-\mathrm{C}(16)-\mathrm{C}(17)-\mathrm{C}(18) & 176.3(4) \\ \mathrm{N}(2)-\mathrm{C}(16)-\mathrm{C}(17)-\mathrm{C}(18) & -5.6(6) \\ \mathrm{O}(1)-\mathrm{C}(16)-\mathrm{C}(17)-\mathrm{C}(19) & -62.8(6) \\ \mathrm{N}(2)-\mathrm{C}(16)-\mathrm{C}(17)-\mathrm{C}(19) & 115.2(5) \\ \mathrm{O}(1)-\mathrm{C}(16)-\mathrm{C}(17)-\mathrm{C}(20) & 54.2(6) \\ \mathrm{N}(2)-\mathrm{C}(16)-\mathrm{C}(17)-\mathrm{C}(20) & -127.8(5)\end{array}$

Symmetry transformations used to generate equivalent atoms:

Table 7. Hydrogen bonds for mo_20150423D_0m [A and deg.].

\begin{tabular}{lllll}
\hline $\mathrm{D}-\mathrm{H} \ldots \mathrm{A}$ & $\mathrm{d}(\mathrm{D}-\mathrm{H})$ & $\mathrm{d}(\mathrm{H} \ldots \mathrm{A})$ & $\mathrm{d}(\mathrm{D} \ldots \mathrm{A})$ & $<(\mathrm{DHA})$ \\
$\mathrm{N}(2)-\mathrm{H}(2) \ldots \mathrm{N}(1)$ & 0.86 & 2.22 & $2.642(6)$ & 109.9 \\
& & & & \\
\hline
\end{tabular}

Symmetry transformations used to generate equivalent atoms: 


\section{Copies of ${ }^{1} \mathrm{H},{ }^{13} \mathrm{C}$ NMR Charts for the Compounds}

$N$-(quinolin-8-yl)pivalamide (1a)
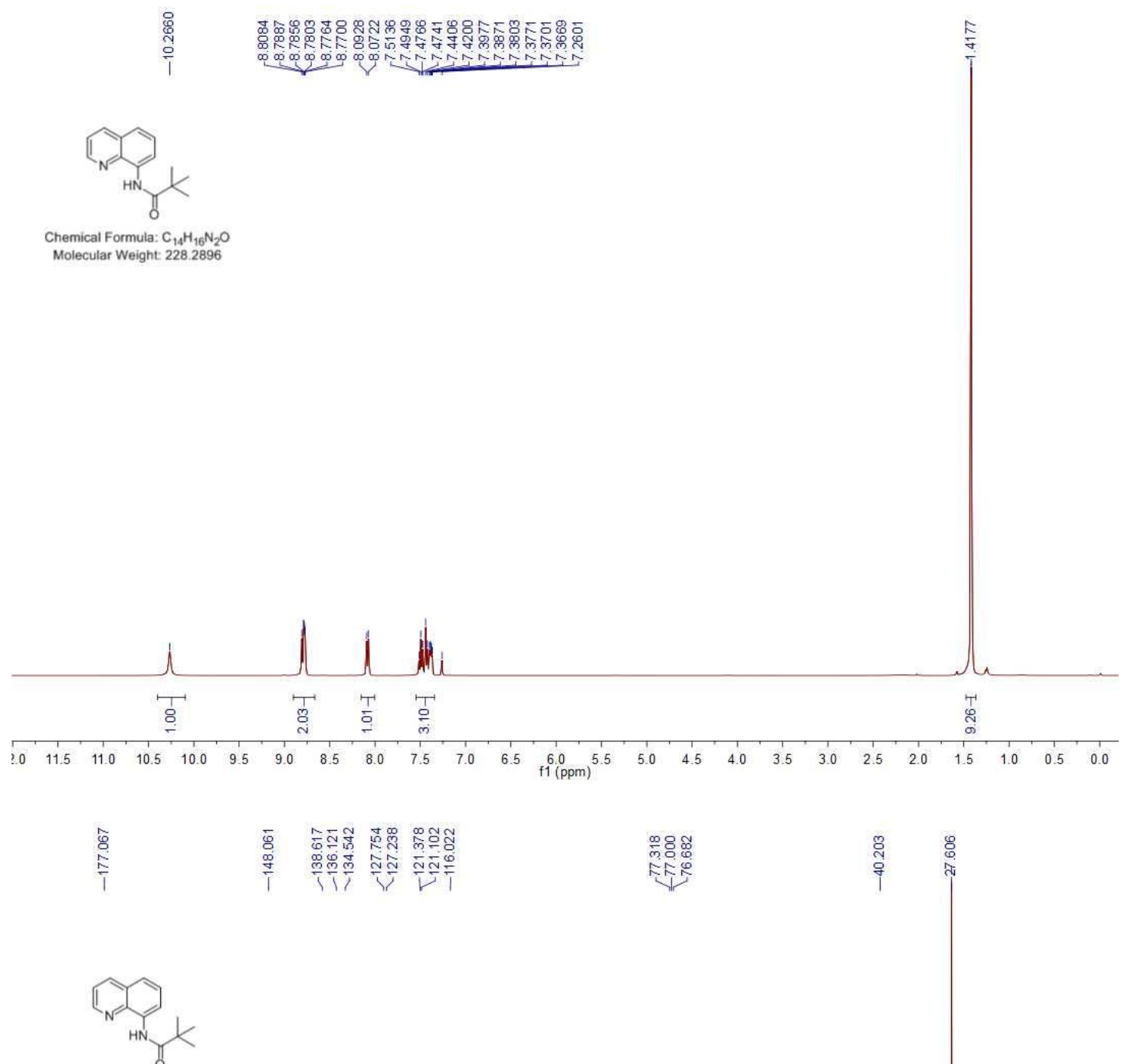

Chemical Formula: $\mathrm{C}_{14} \mathrm{H}_{16} \mathrm{~N}_{2} \mathrm{O}$

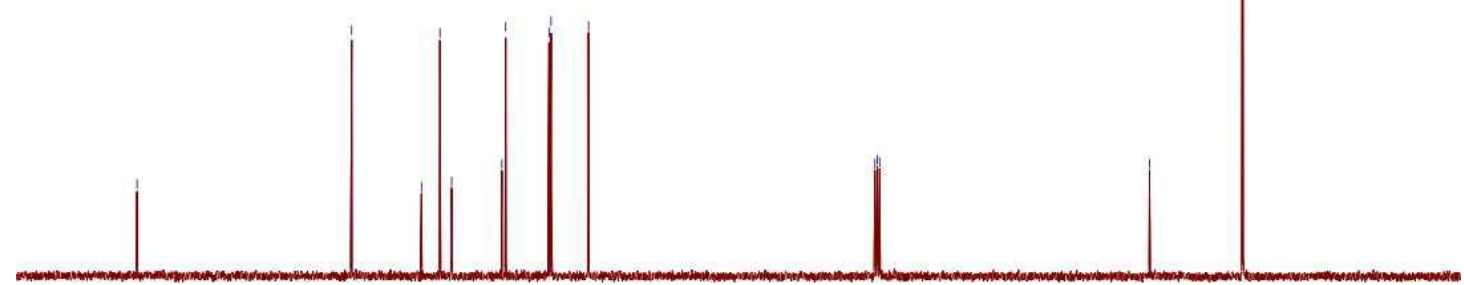


$N$-(quinolin-5-yl)pivalamide (1aa)
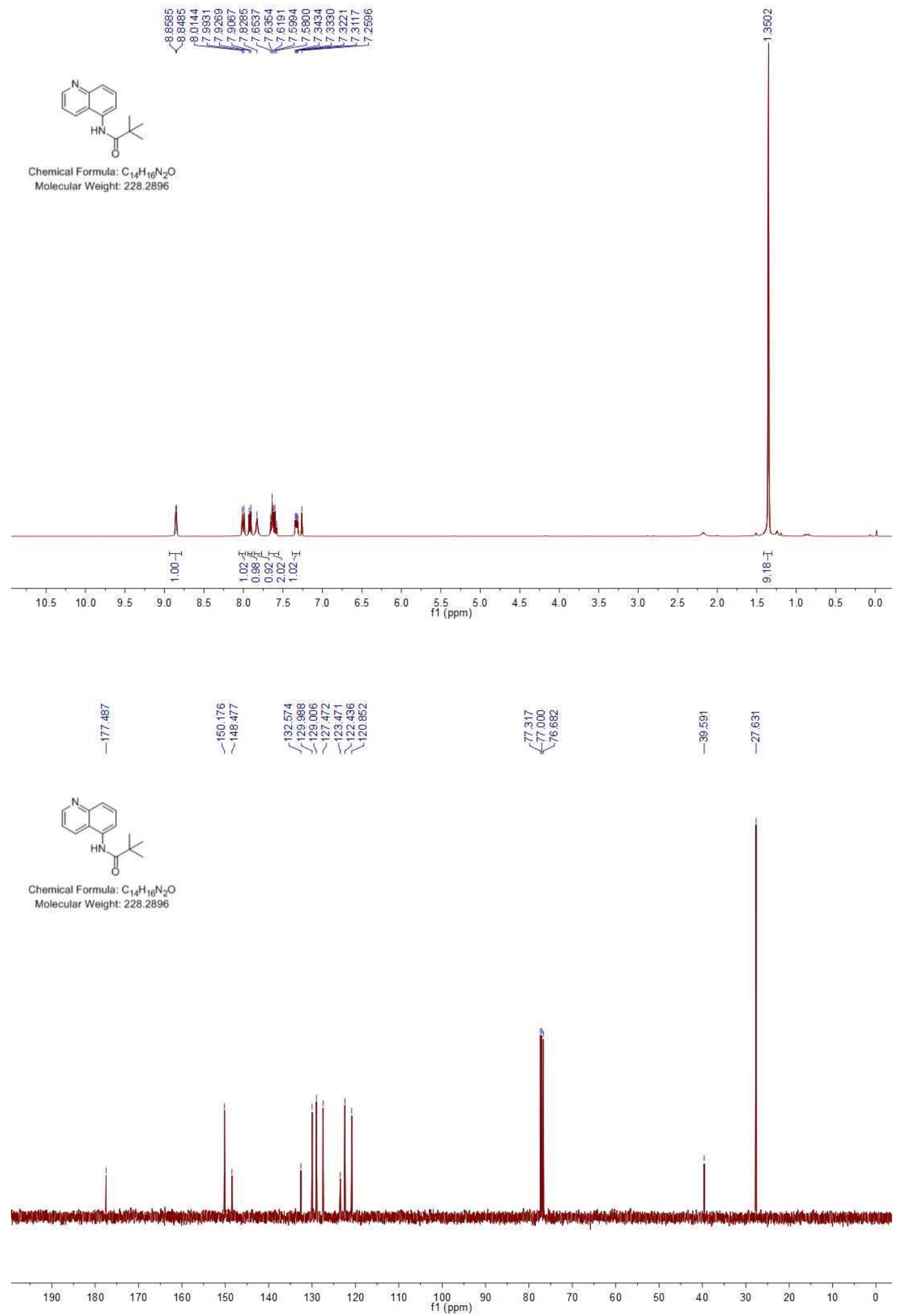
$N$-(quinolin-8-yl)acetamide (1b)

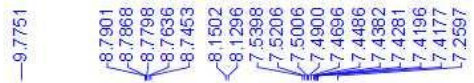

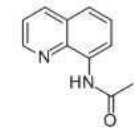

Chemical Formula: $\mathrm{C}_{11} \mathrm{H}_{10} \mathrm{~N}_{2} \mathrm{O}$

Molecular Weight: 1862099

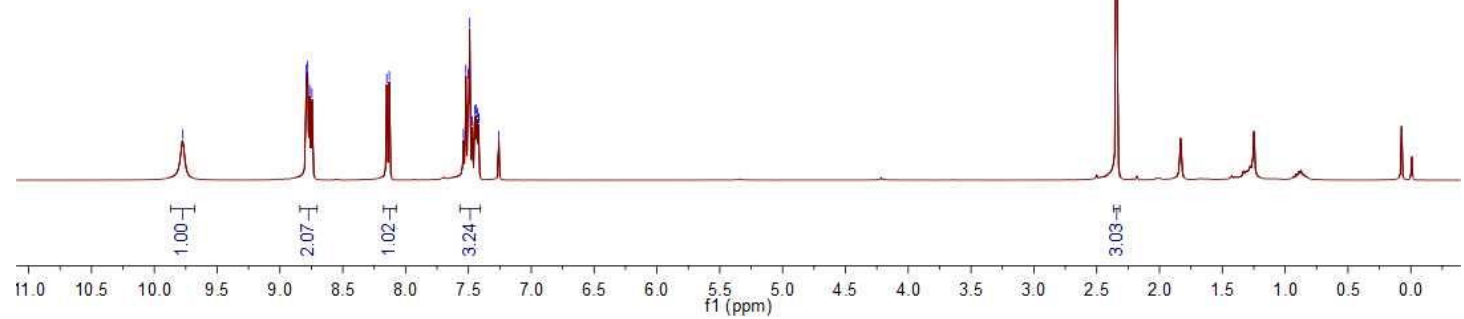

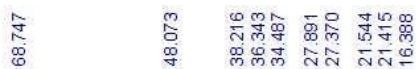

市

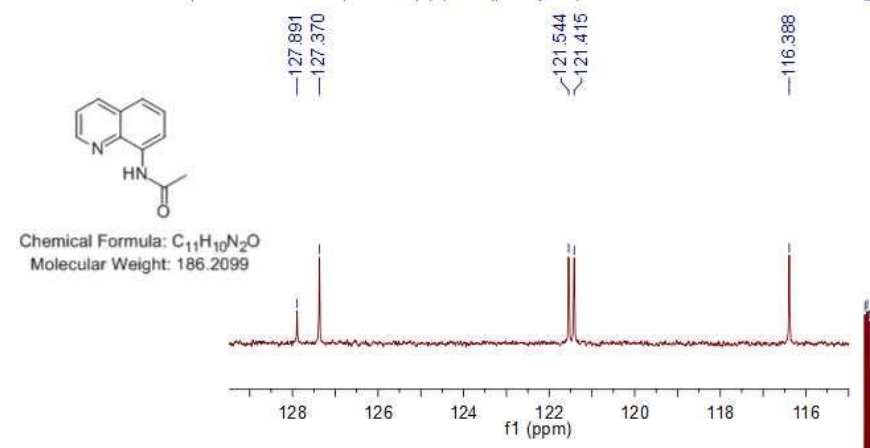

mo:

党

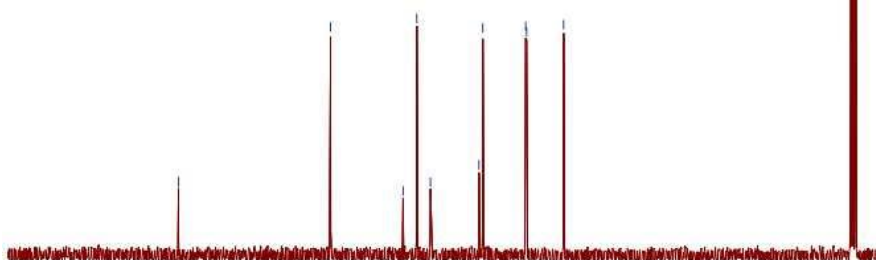

$\begin{array}{llllllllll}190 & 180 & 170 & 160 & 150 & 140 & 130 & 120 & 110 & 100\end{array}$ 
2,2-dimethyl- $N$-(quinolin-8-yl)butanamide (1c)

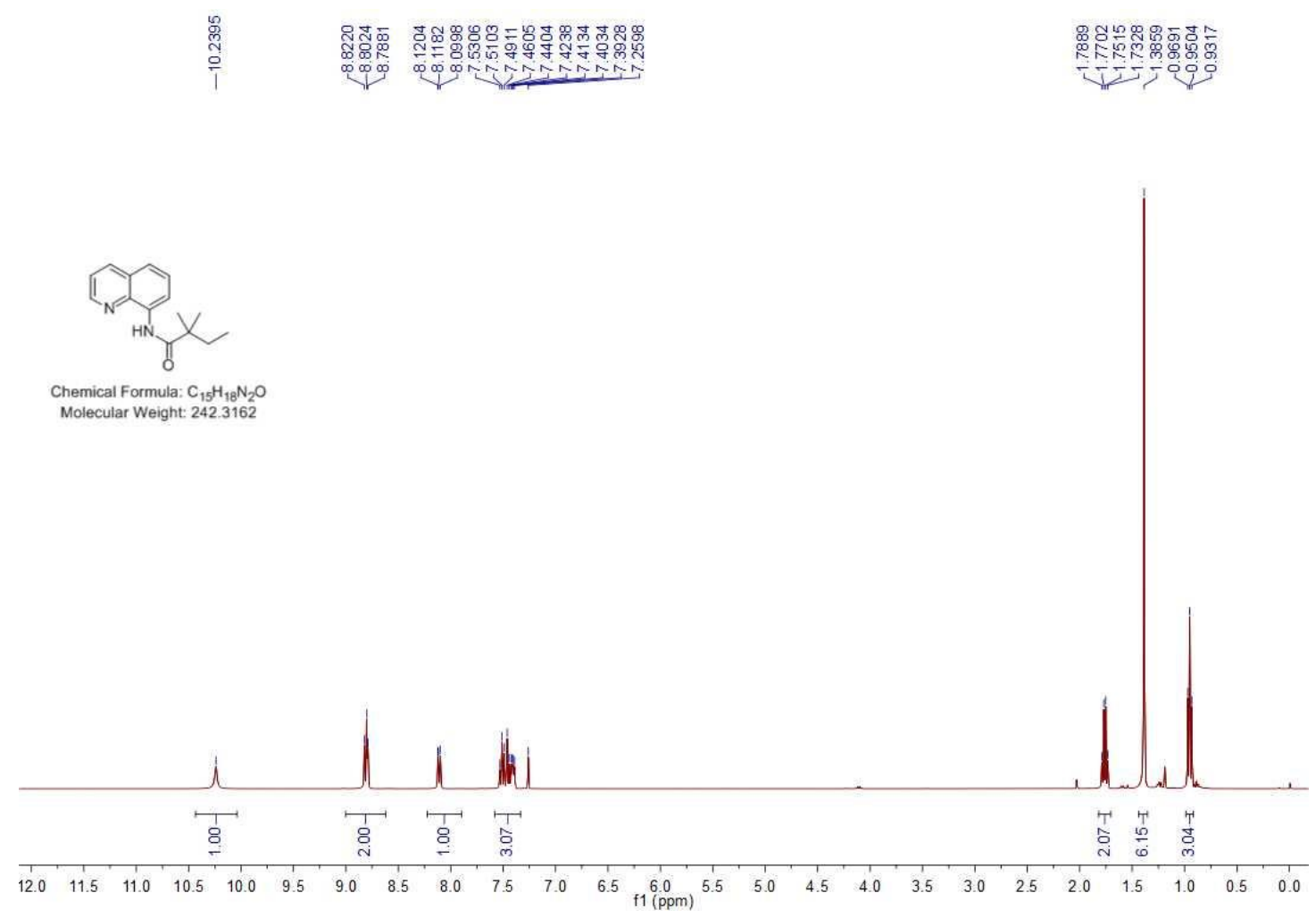

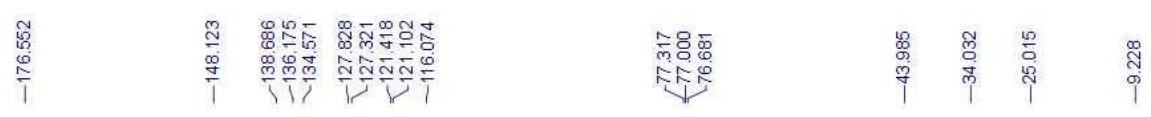

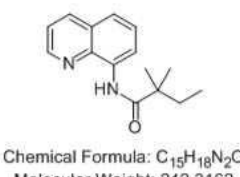

Molecular Weight: 2423162

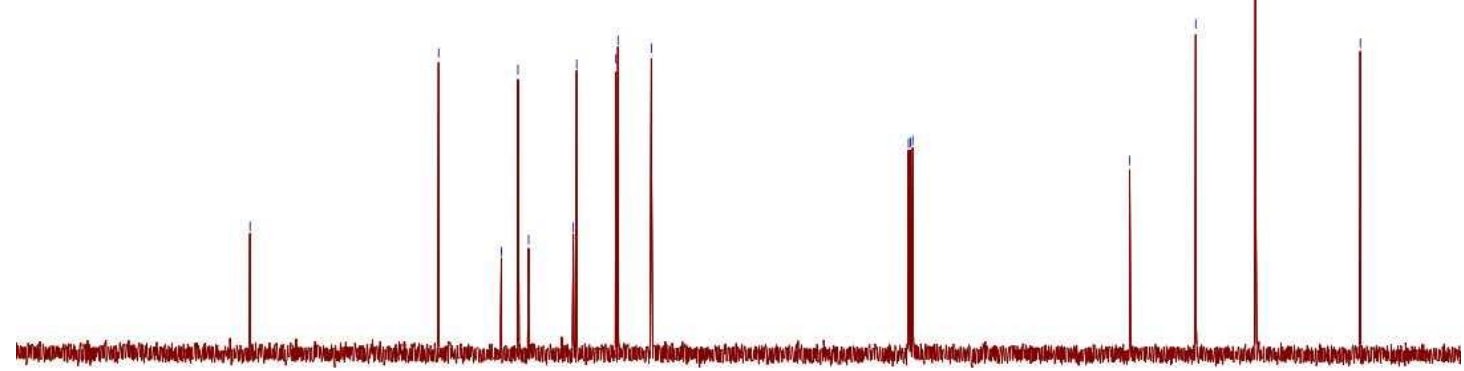

$\begin{array}{llllllllllllllllllllllllll} & 210 & 200 & 190 & 180 & 170 & 160 & 150 & 140 & 130 & 120 & 110 & 100 & 90 & 80 & 70 & 60 & 50 & 40 & 30 & 20 & 10 & 0\end{array}$ 
2-ethyl- $N$-(quinolin-8-yl)butanamide (1d)

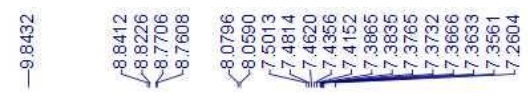

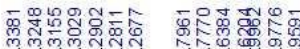

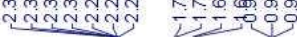

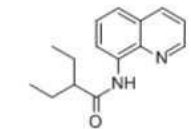

Chemical Formula: $\mathrm{C}_{15} \mathrm{H}_{10} \mathrm{~N}_{2} \mathrm{O}$ Molecular Weight: 242.3162
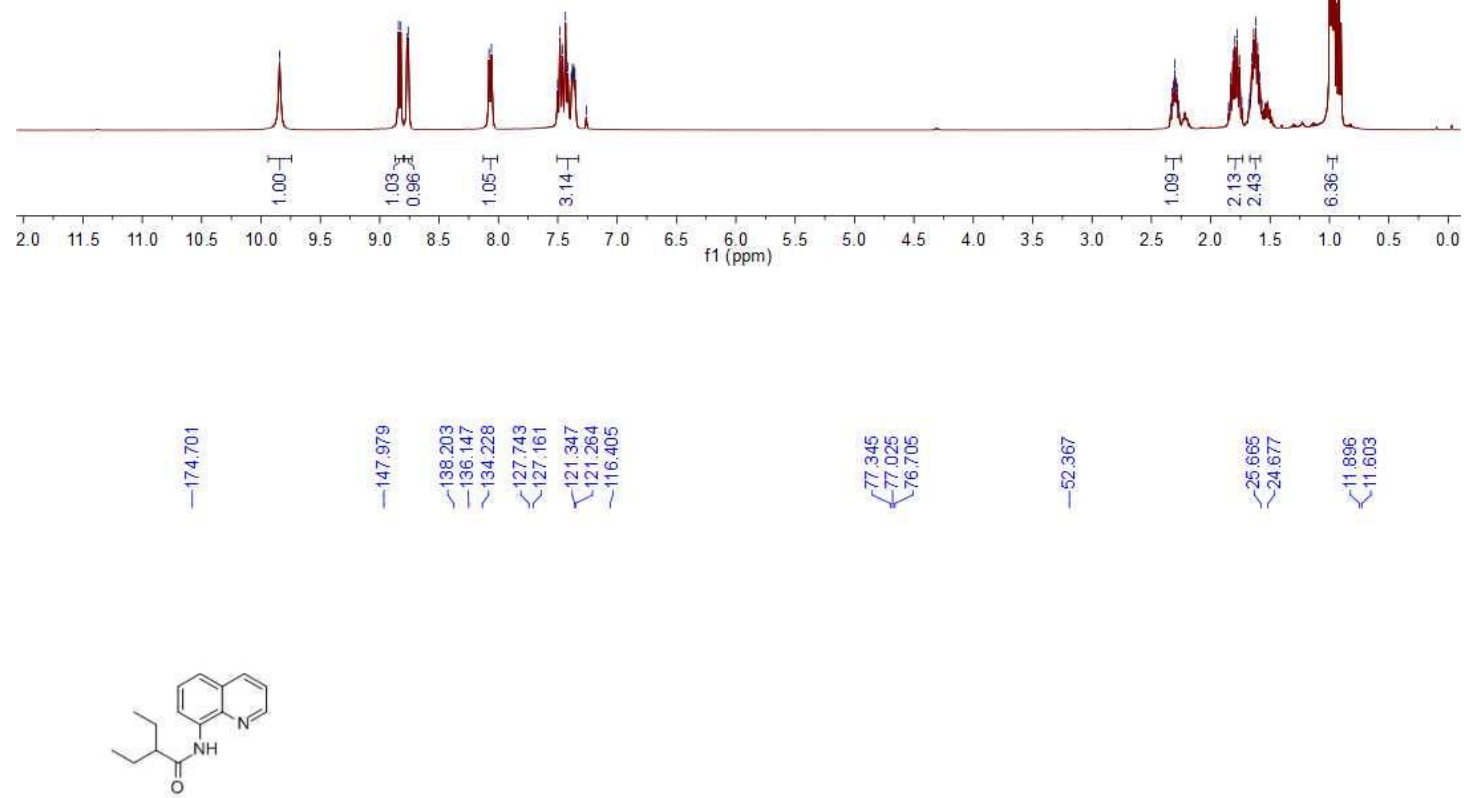

Chemical Formula: $\mathrm{C}_{15} \mathrm{H}_{18} \mathrm{~N}_{2} \mathrm{O}$

Molecular Weight 242.3162

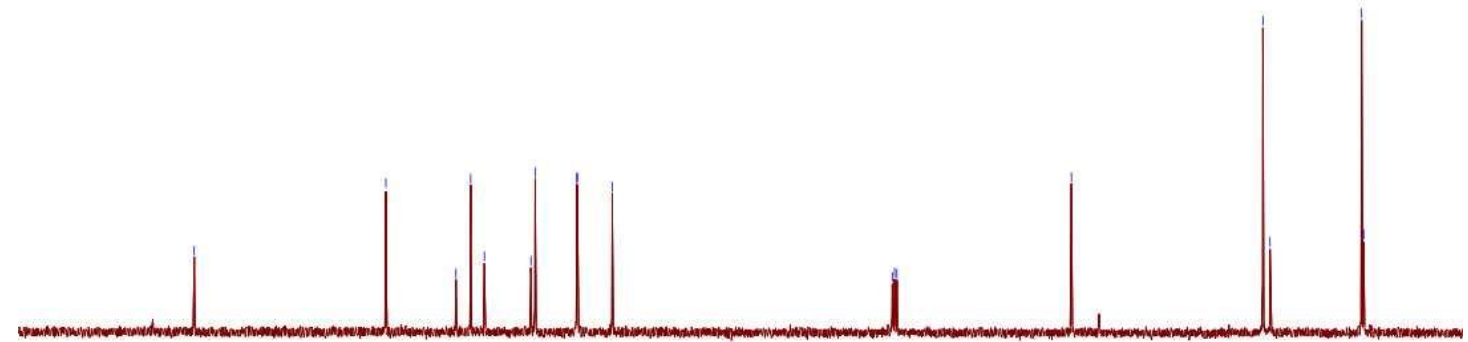

$\begin{array}{lllll}190 & 180 & 170 & 160 & 150\end{array}$ 100
$\mathrm{f} 1(\mathrm{ppm})$ 
$N$-(quinolin-8-yl)cyclopentanecarboxamide (1e)
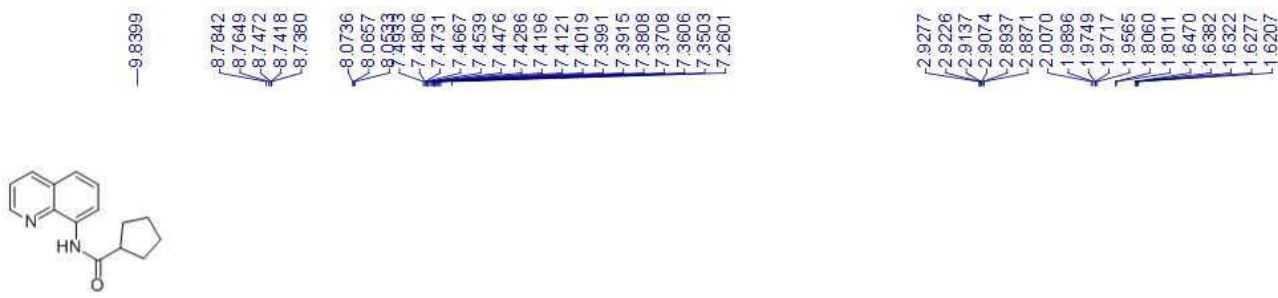

Chemical Formula: $\mathrm{C}_{15} \mathrm{H}_{16} \mathrm{~N}_{2} \mathrm{O}$

Molecular Weight: 240.3003

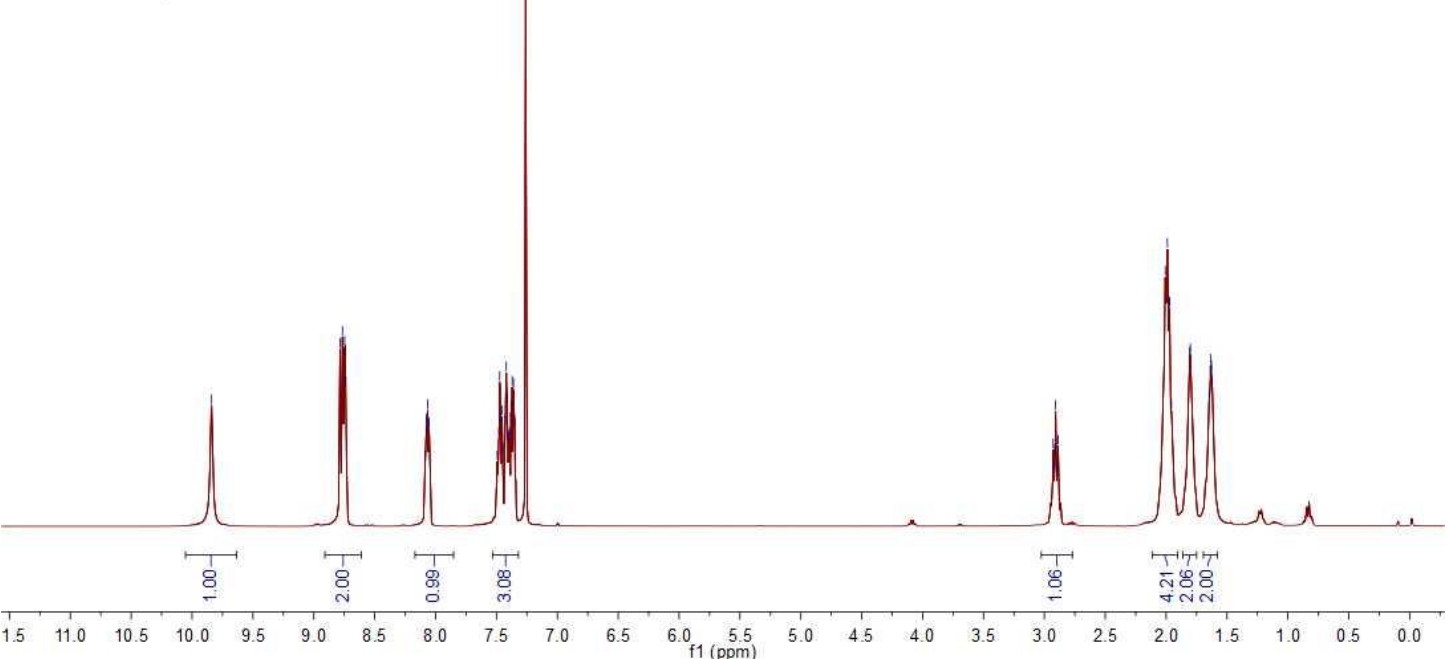

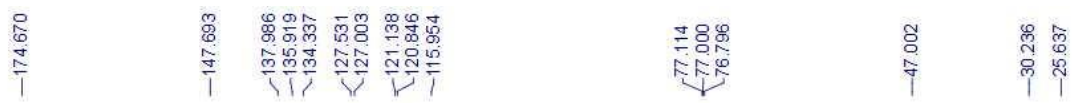

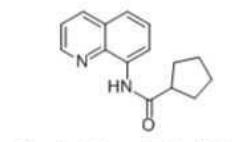

Chemical Formula: $\mathrm{C}_{15} \mathrm{H}_{16} \mathrm{~N}_{2} \mathrm{O}$

Molecular Weight: 240.3003

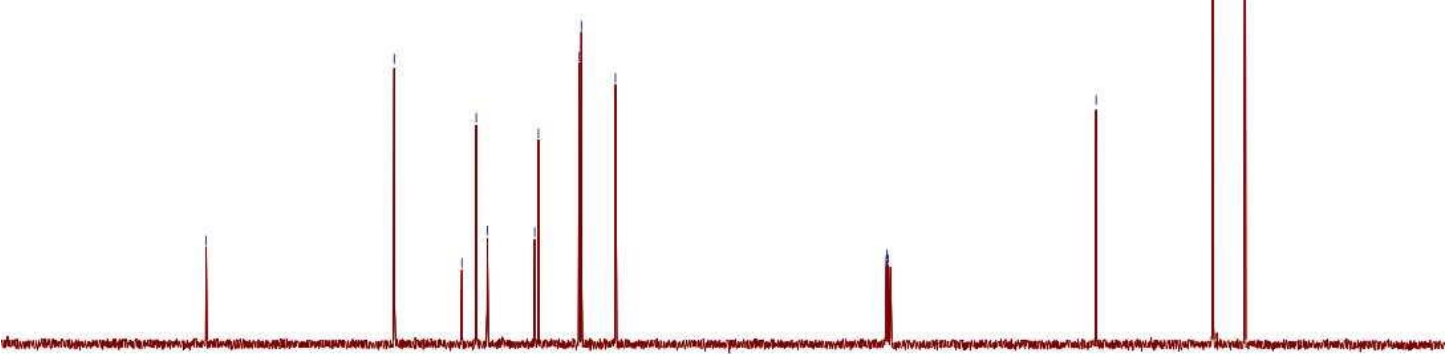

$\begin{array}{lllllllllllllllllllllll}200 & 190 & 180 & 170 & 160 & 150 & 140 & 130 & 120 & 110 & 100 & 90 & 80 & 70 & 60 & 50 & 40 & 30 & 20 & 10 & 0\end{array}$ 
1-methyl- $N$-(quinolin-8-yl)cyclohexanecarboxamide (1f)

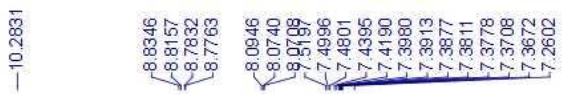

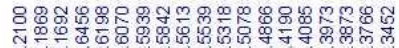

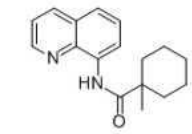

Chemical Formula: $\mathrm{C}_{17} \mathrm{H}_{20} \mathrm{~N}_{2} \mathrm{O}$

Molecular Weight: 268.3535

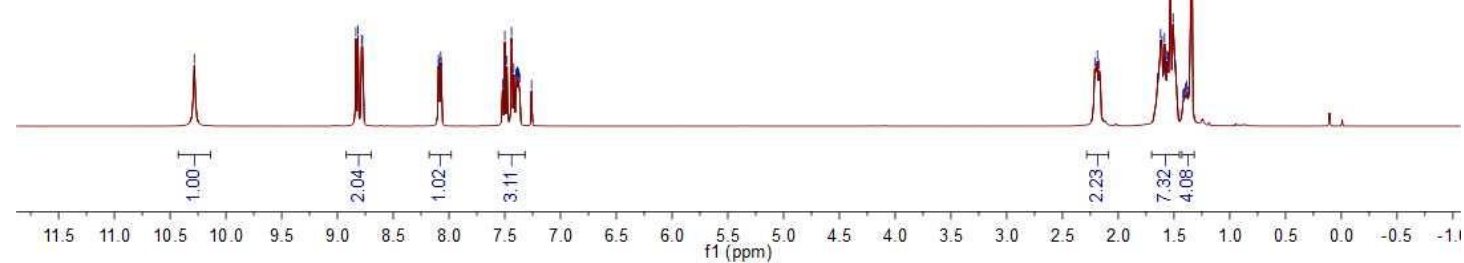

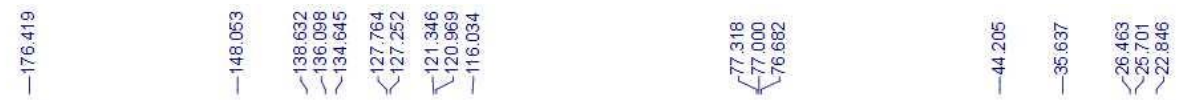

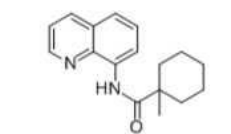

Chemical Formula: $\mathrm{C}_{17} \mathrm{H}_{20} \mathrm{~N}_{2} \mathrm{O}$

Molecular Weight: 268.3535

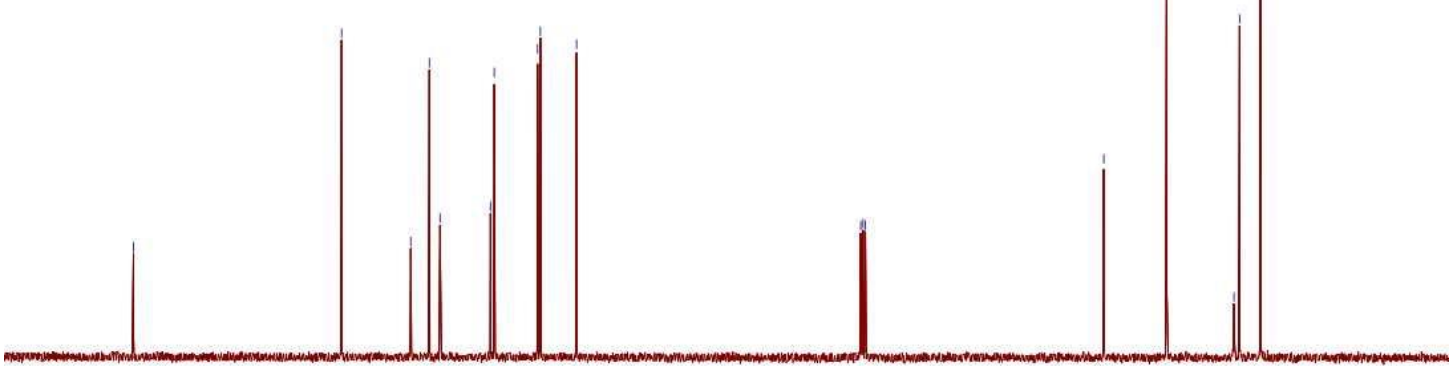

$\begin{array}{llllllllll}190 & 180 & 170 & 160 & 150 & 140 & 130 & 120 & 110 & \underset{\mathrm{f} 1(\mathrm{ppm})}{100}\end{array}$

$\begin{array}{lllllllll}80 & 70 & 60 & 50 & 40 & 30 & 20 & 10 & 0\end{array}$ 
$N$-(quinolin-8-yl)octanamide (1g)

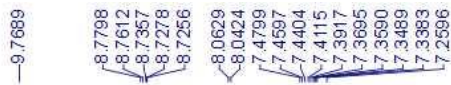

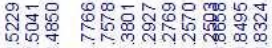

Nin = = Ti0

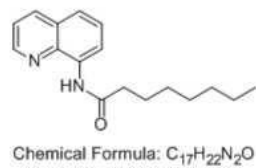

Momical Formula. $\mathrm{C}_{17} \mathrm{H}_{22} \mathrm{~N}_{2} \mathrm{O}$

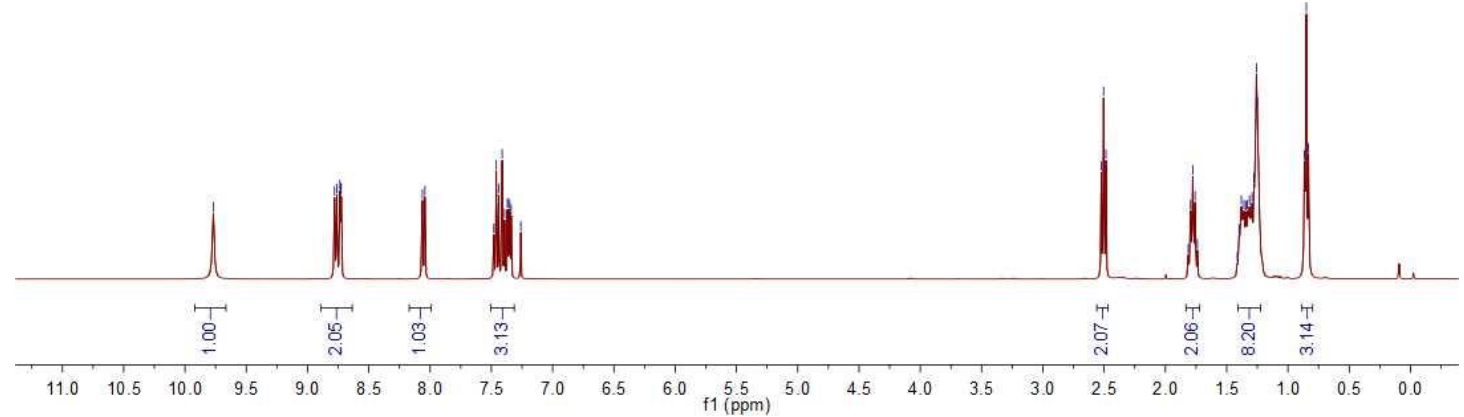

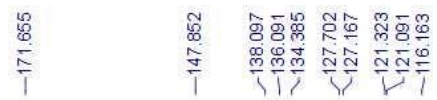

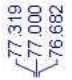

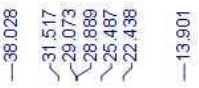

(1)

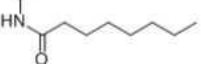

Chemical Formula: $\mathrm{C}_{17} \mathrm{H}_{22} \mathrm{~N}_{2} \mathrm{O}$

Molecular Weight 270.3694

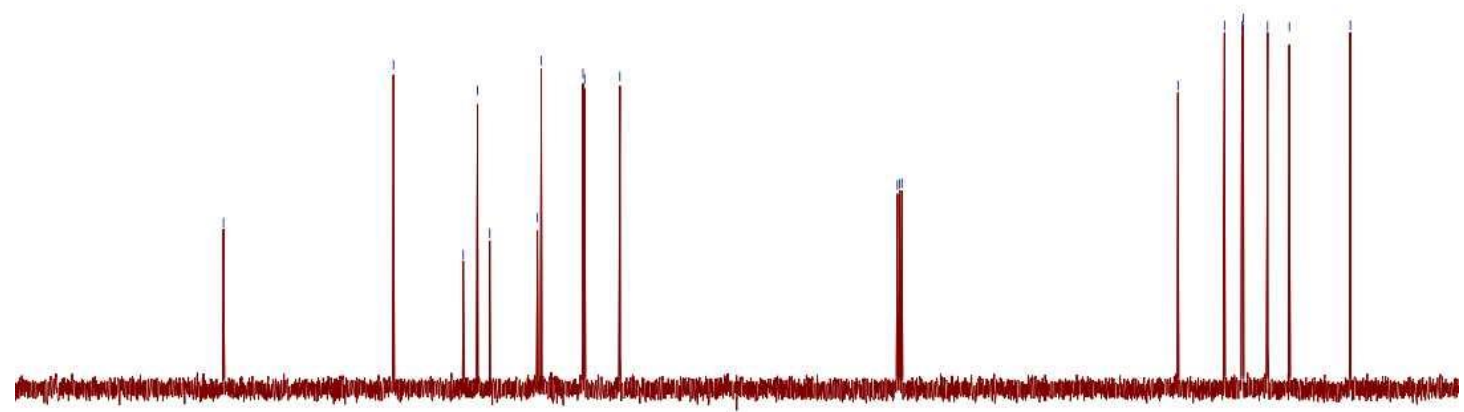

$\begin{array}{llllllllllllllllllllllll}00 & 190 & 180 & 170 & 160 & 150 & 140 & 130 & 120 & 110 & 100 & 10 & 80 & 70 & 60 & 50 & 40 & 30 & 20 & 10 & 0\end{array}$ 
11-bromo- $N$-(quinolin-8-yl)undecanamide (1h)

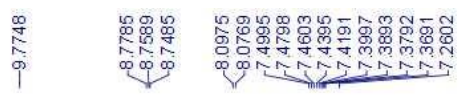

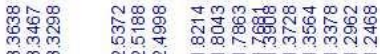

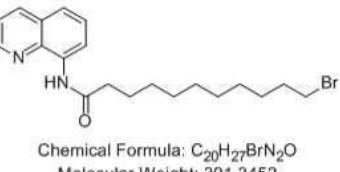

Molecular Weight: 391.3452
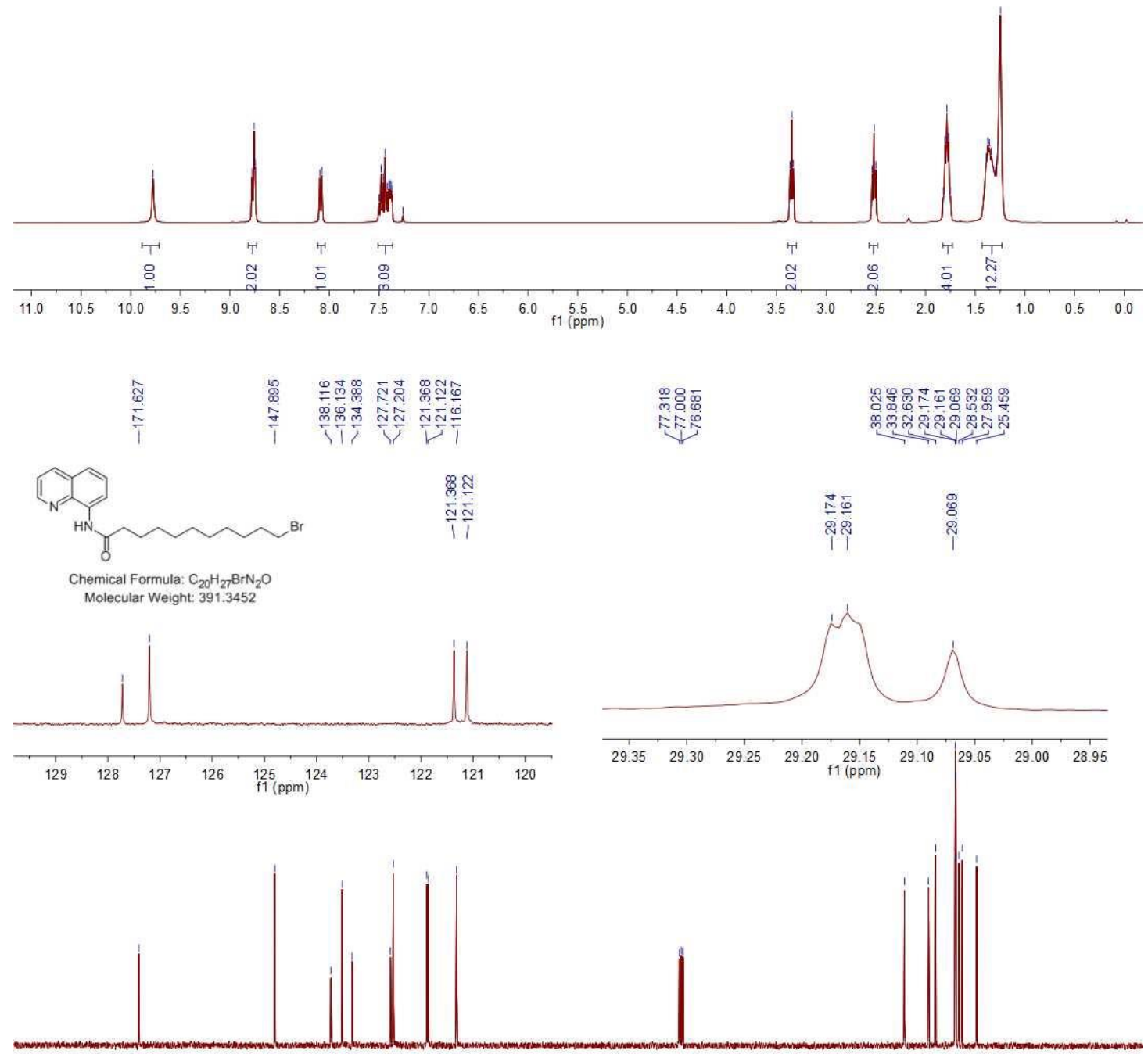

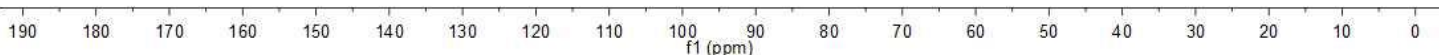


$N$-(5-(phenylthio)quinolin-8-yl)pivalamide (3a)
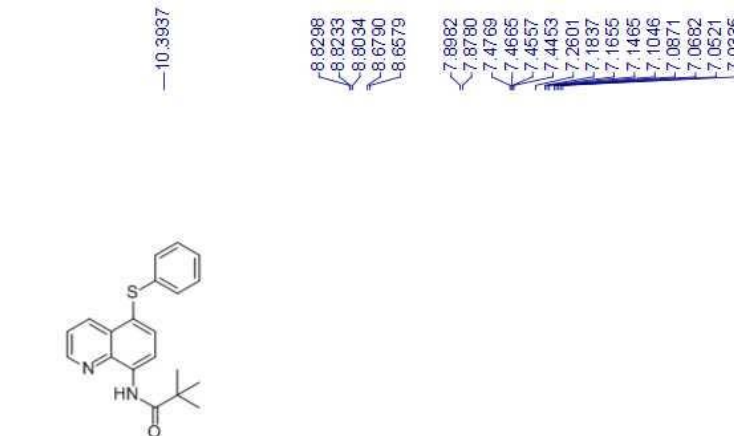

Chemical Formula: $\mathrm{C}_{20} \mathrm{H}_{20} \mathrm{~N}_{2} \mathrm{O}$ Molecular Weight: 336.4506
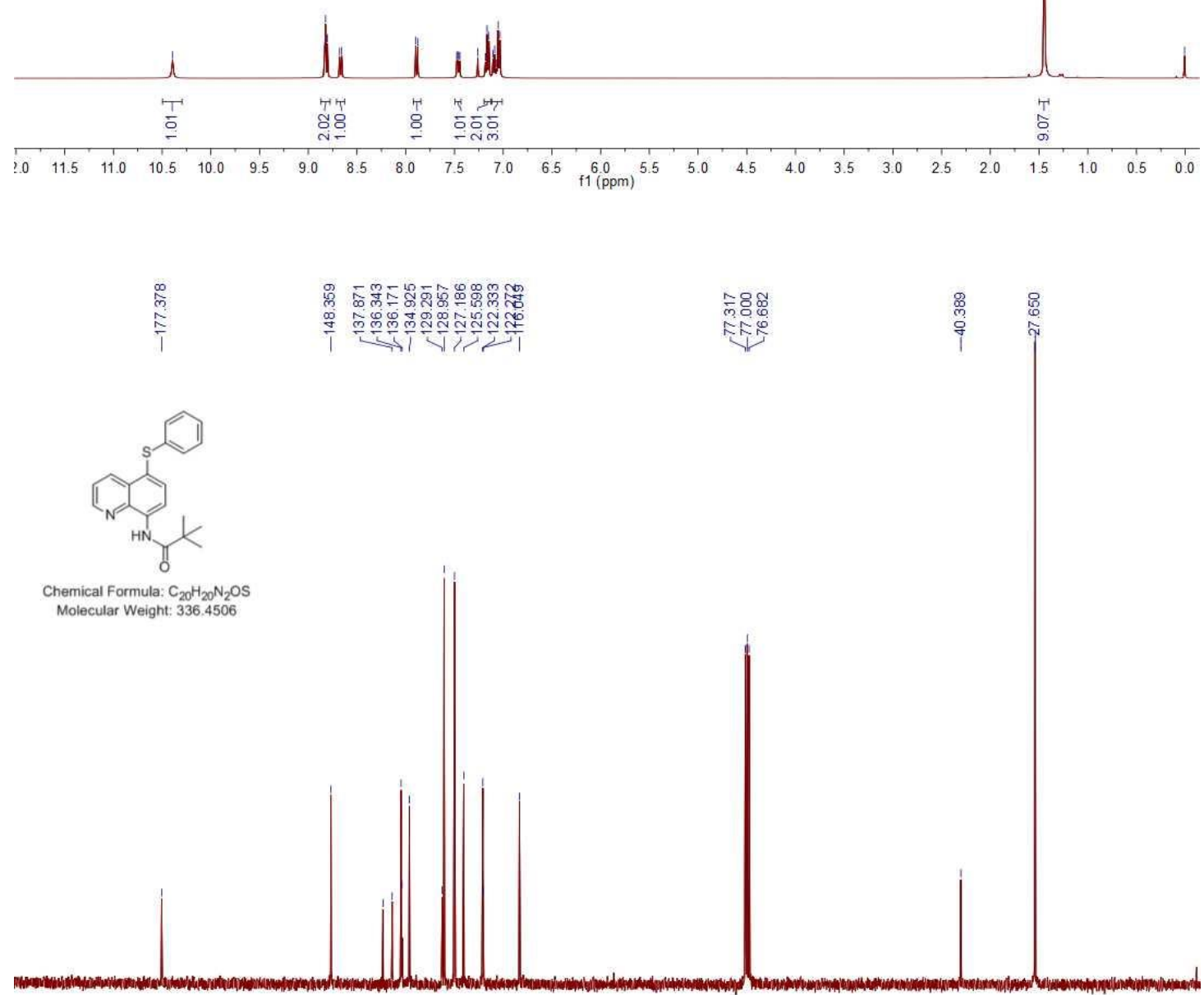

$\begin{array}{lllllllllllllllllllllll}200 & 190 & 180 & 170 & 160 & 150 & 140 & 130 & 120 & 110 & \begin{array}{l}100 \\ \mathrm{f} 1(\mathrm{ppm})\end{array} & 90 & 80 & 70 & 60 & 50 & 40 & 30 & 20 & 10 & 0\end{array}$ 
$N$-(5-bromoquinolin-8-yl)pivalamide (3a')
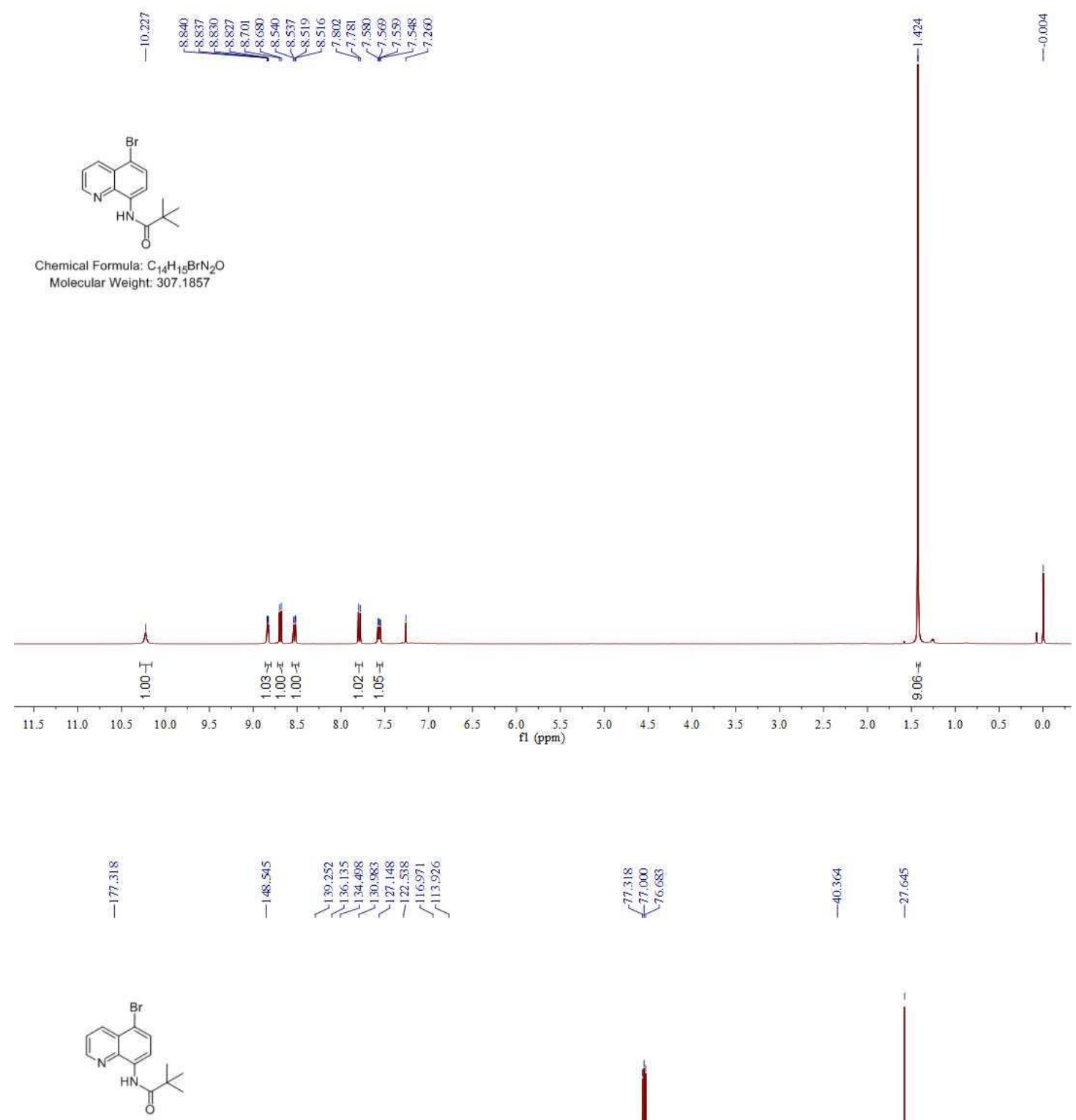

Molecular Weight: 307.1857
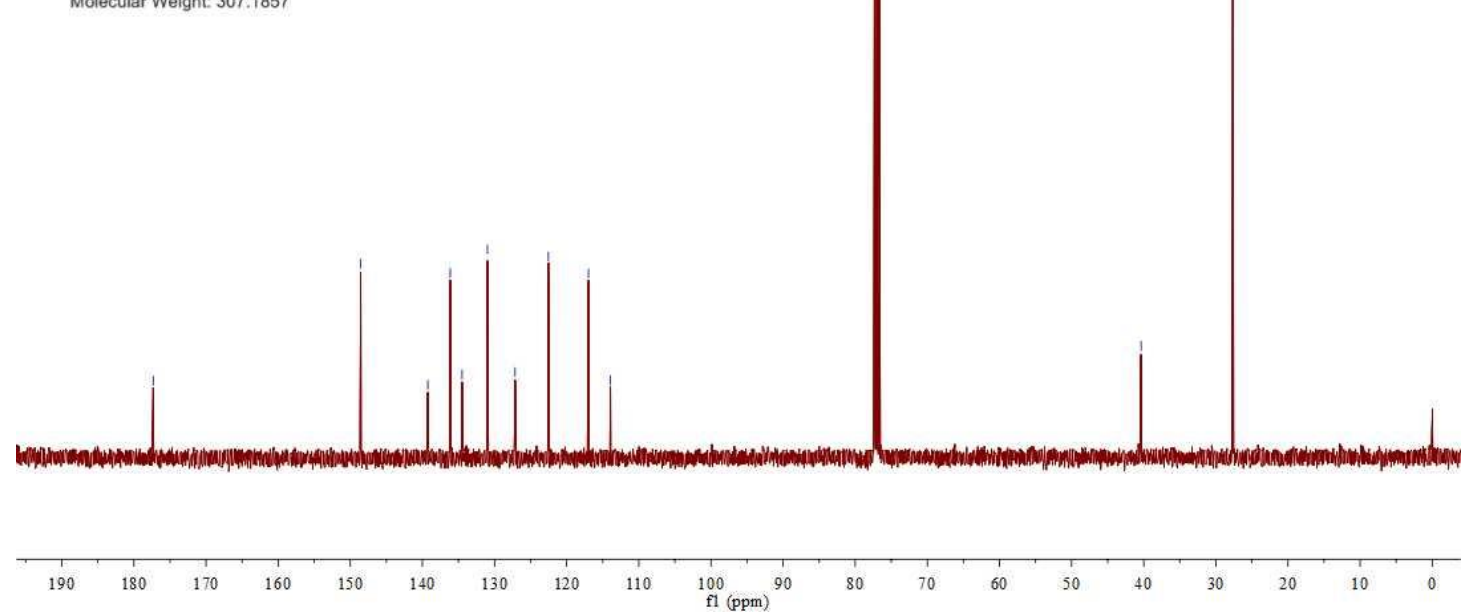
$N$-(5-(p-tolylthio)quinolin-8-yl)pivalamide (3b)
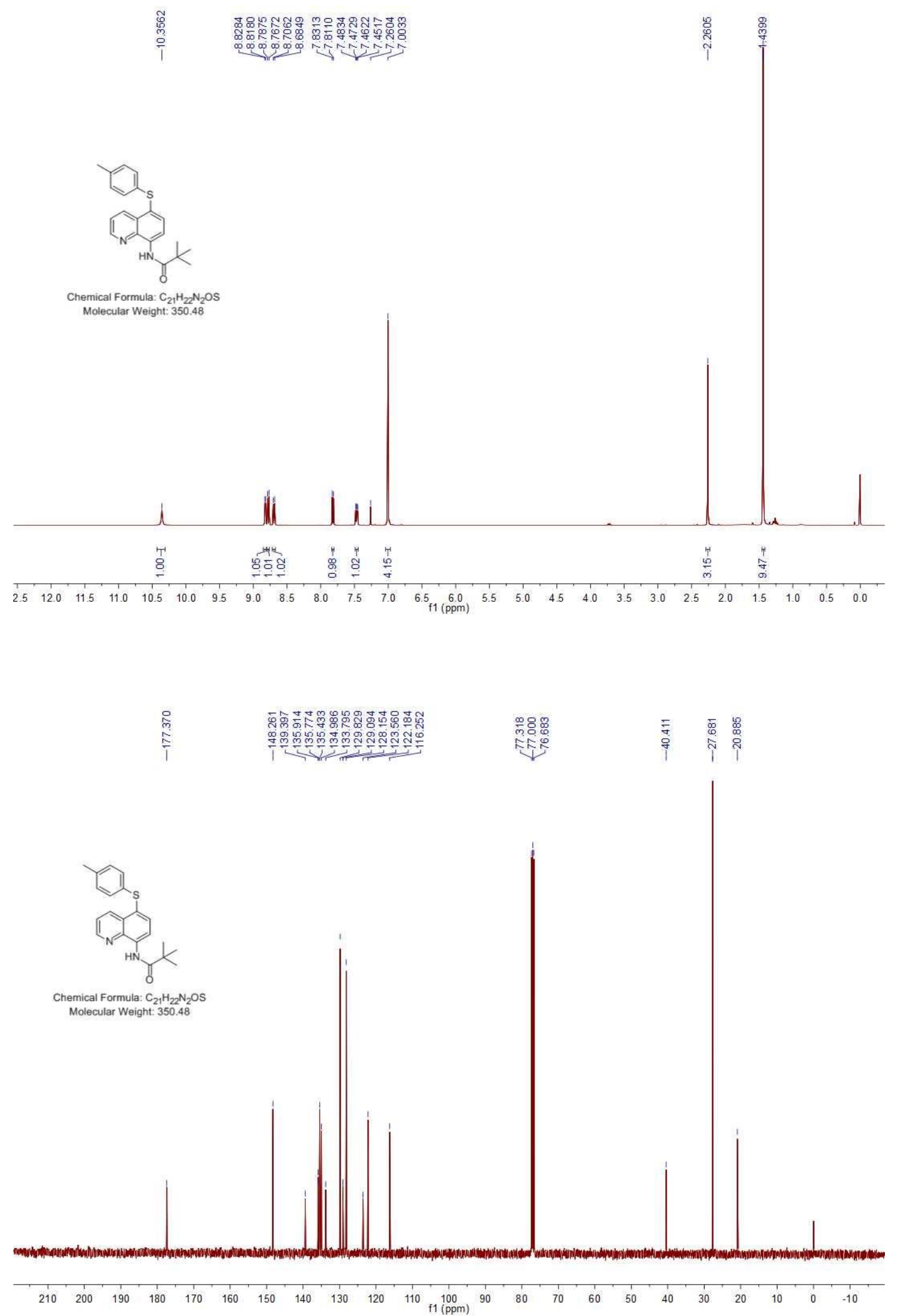
$N$-(5-((4-methoxyphenyl)thio)quinolin-8-yl)pivalamide (Bc)

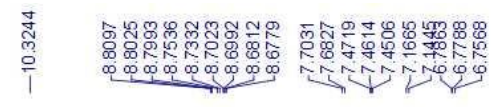

$\underset{\substack{8 \\ 0}}{\substack{1 \\ 0}}$

$\underset{\substack{\mathscr{g} \\ \hdashline}}{i}$

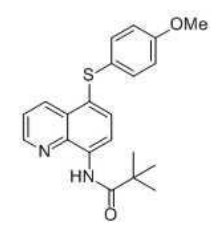

Chemical Formula: $\mathrm{C}_{21} \mathrm{H}_{22} \mathrm{~N}_{2} \mathrm{O}_{2} \mathrm{~S}$
Molecular Weight: 366.48

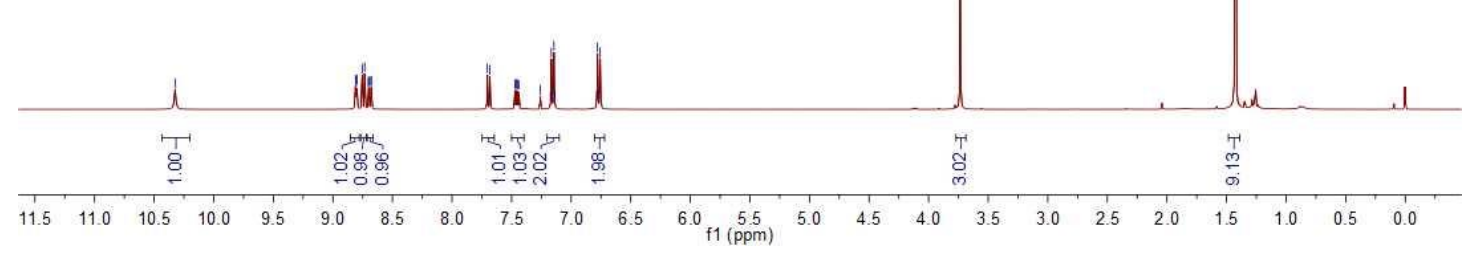

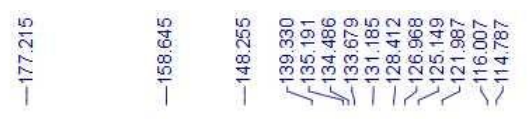

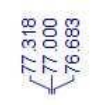

帝离亭

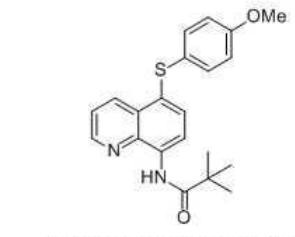
Chemical Formula: $\mathrm{C}_{21} \mathrm{H}_{22} \mathrm{~N}_{2} \mathrm{O}_{2} \mathrm{~S}$
Molecular Weight: 366.48
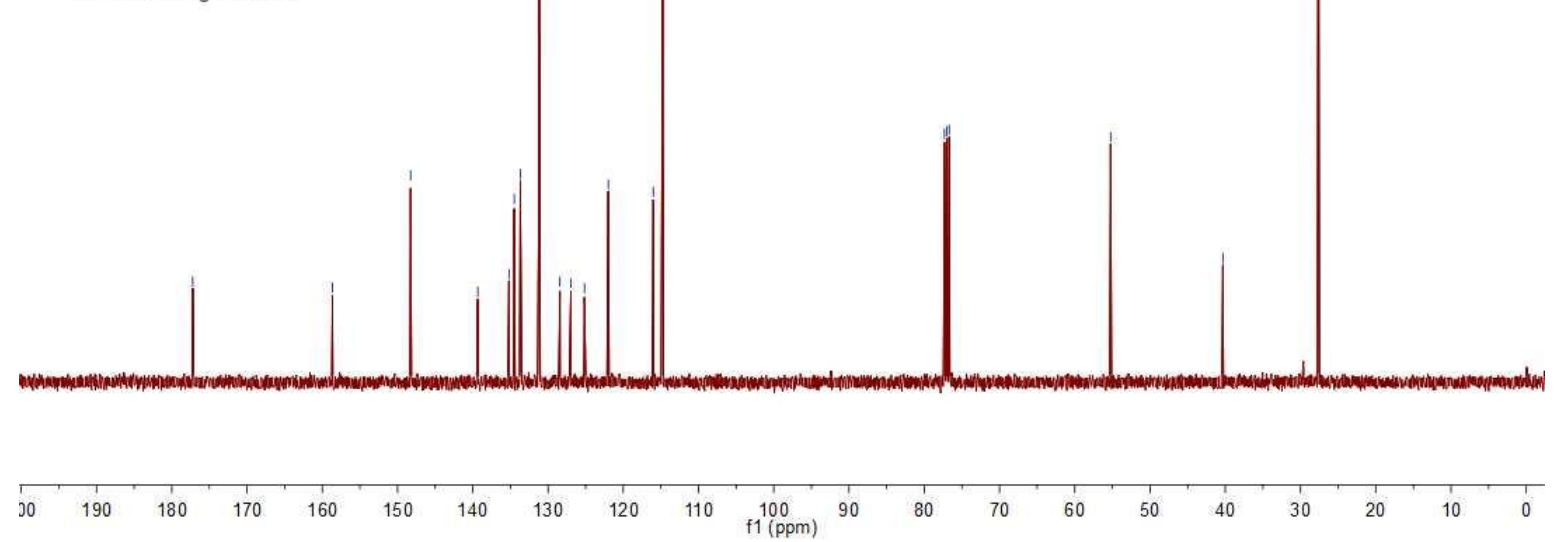

S41 
$N$-(5-((4-chlorophenyl)thio)quinolin-8-yl)pivalamide (3d)
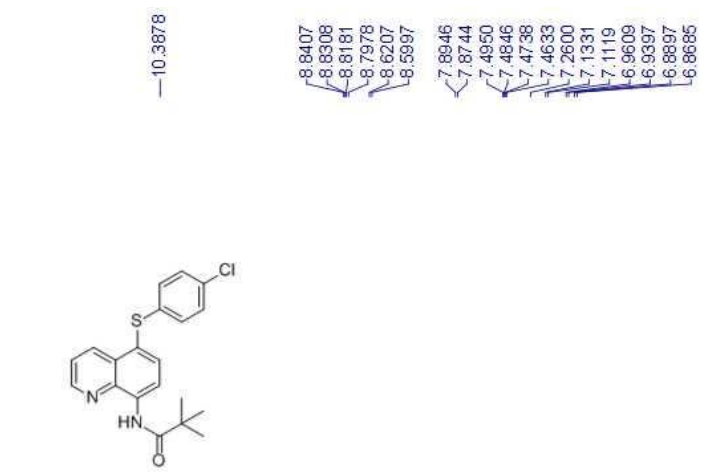

Chemical Formula: $\mathrm{C}_{20} \mathrm{H}_{19} \mathrm{CIN}_{2} \mathrm{OS}$

Molecular Weight: 370.90
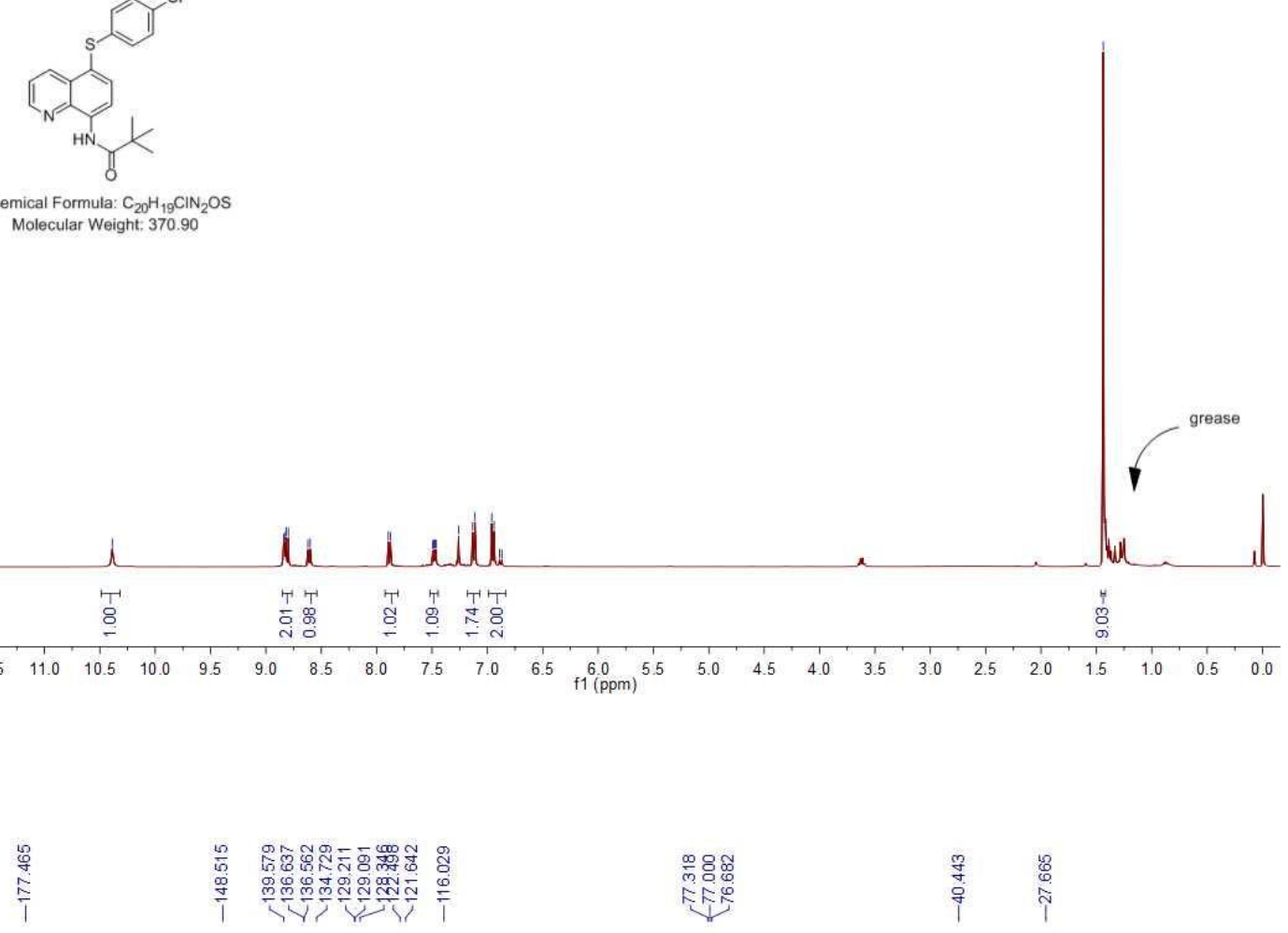

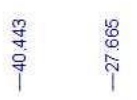

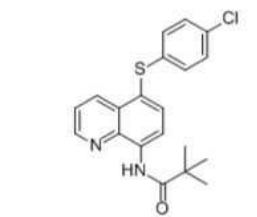

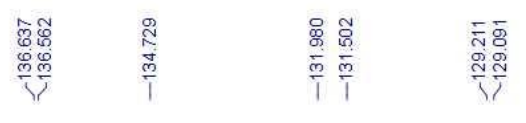

Chemical Formula: $\mathrm{C}_{20} \mathrm{H}_{19} \mathrm{CIN}_{2} \mathrm{OS}$

Molecular Weight: 370.90

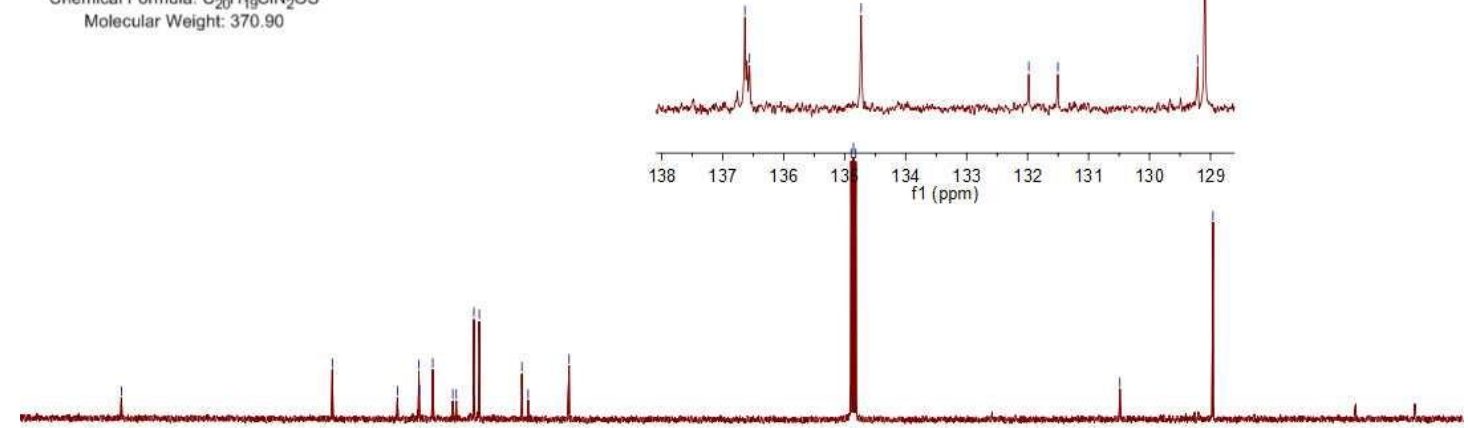

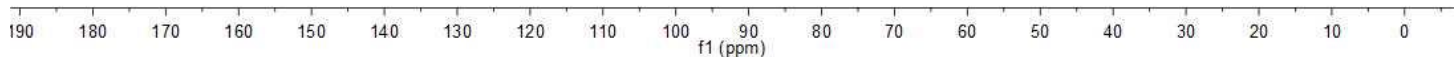


$N$-(5-((4-bromophenyl)thio)quinolin-8-yl)pivalamide (3e)

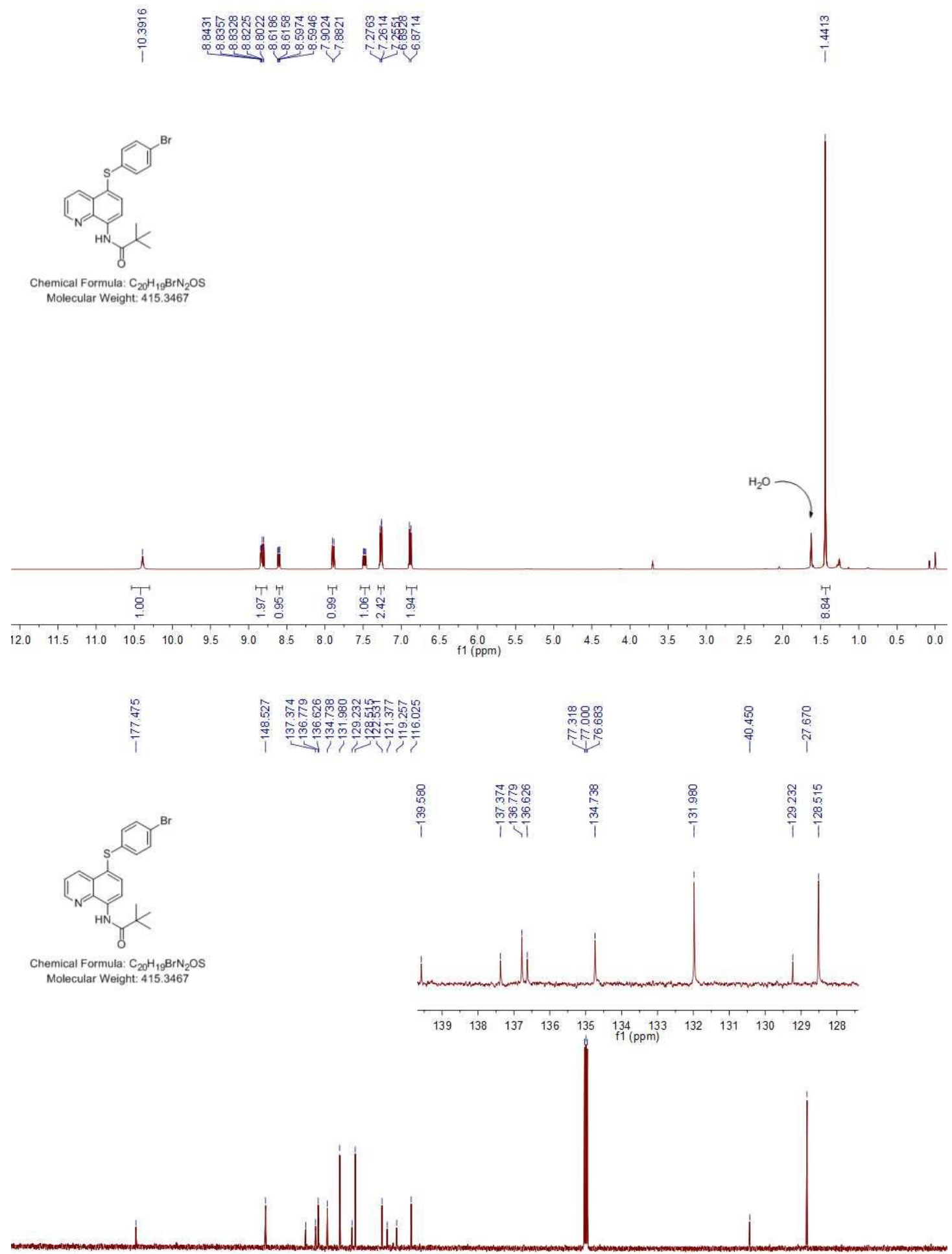

Chemical Formula: $\mathrm{C}_{20} \mathrm{H}_{19} \mathrm{Br} \mathrm{N}_{2} \mathrm{OS}$

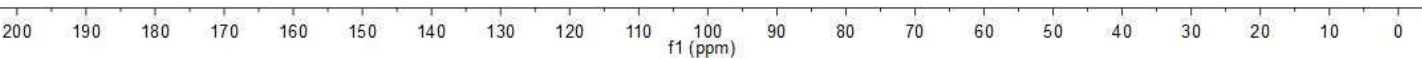


$N$-(5-((4-nitrophenyl)thio)quinolin-8-yl)pivalamide (3f)
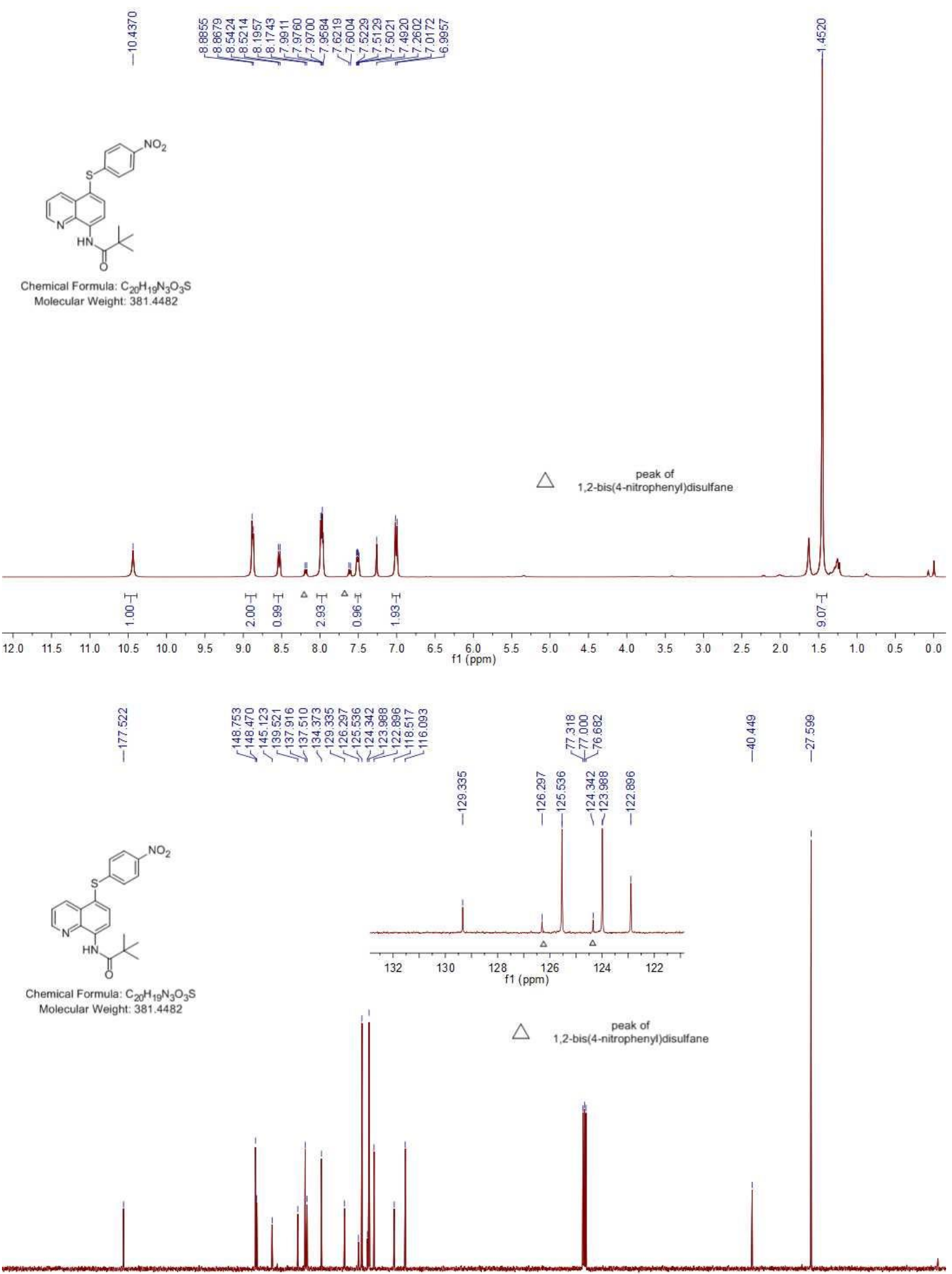

$\begin{array}{llllllllllllllllllllllllll}1 & 1 & 190 & 180 & 170 & 160 & 150 & 140 & 130 & 120 & 110 & 100 & 90 & 80 & 70 & 60 & 50 & 40 & 30 & 20 & 10 & 0\end{array}$ 
1,2-bis(4-nitrophenyl)disulfane
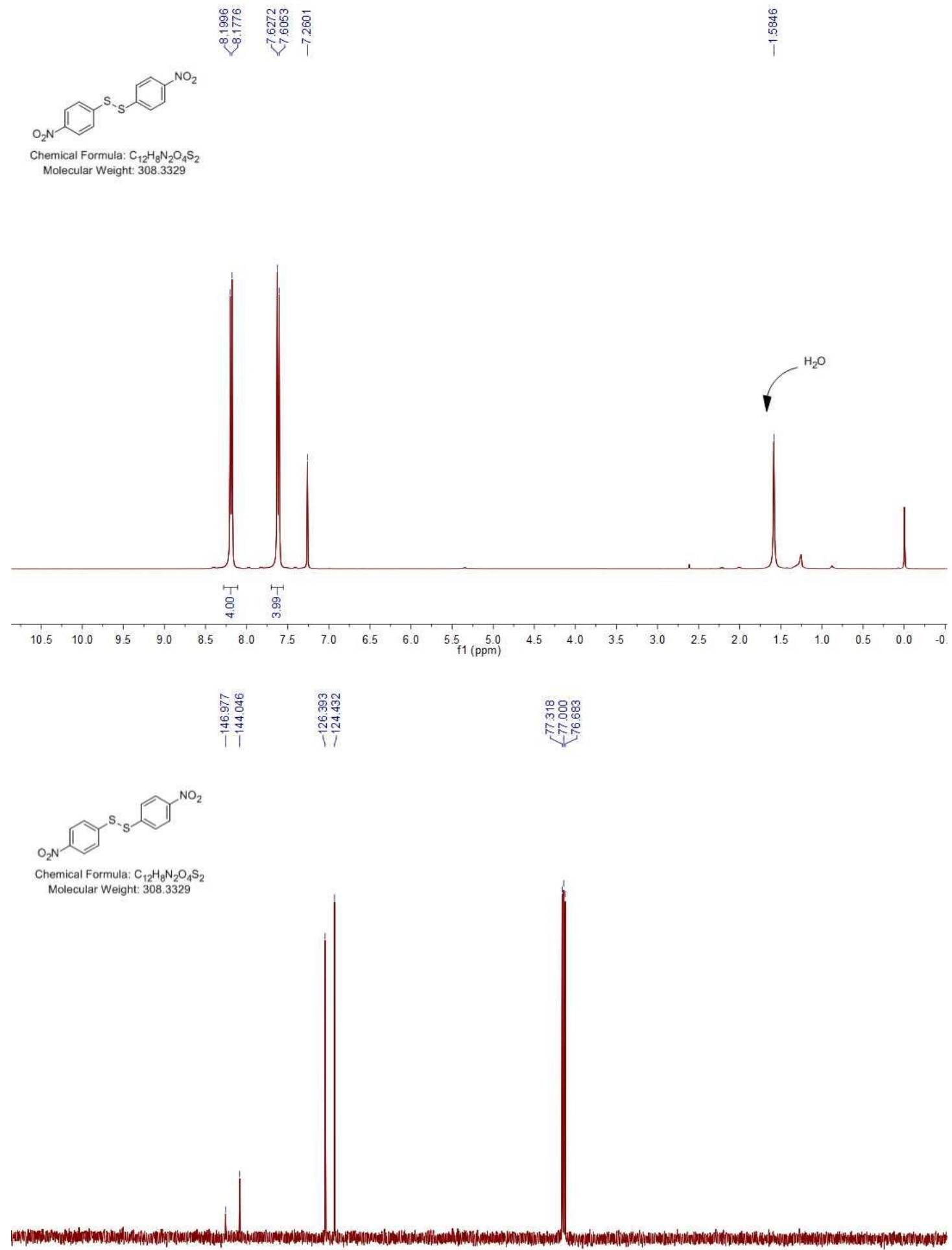

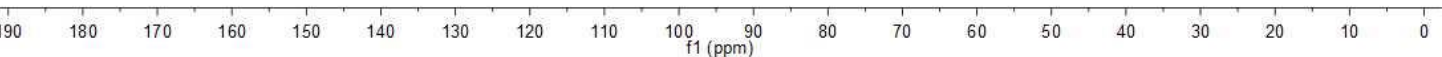


$N-(5-((3-n i t r o p h e n y l)$ thio)quinolin-8-yl)pivalamide (3g)
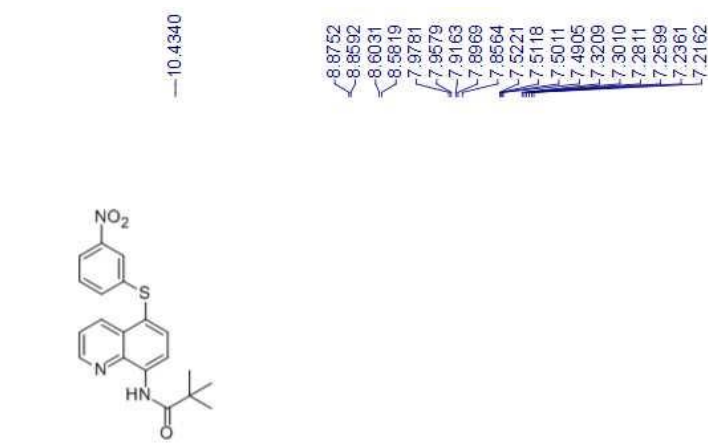

Chemical Formula: $\mathrm{C}_{20} \mathrm{H}_{19} \mathrm{~N}_{3} \mathrm{O}_{3} \mathrm{~S}$

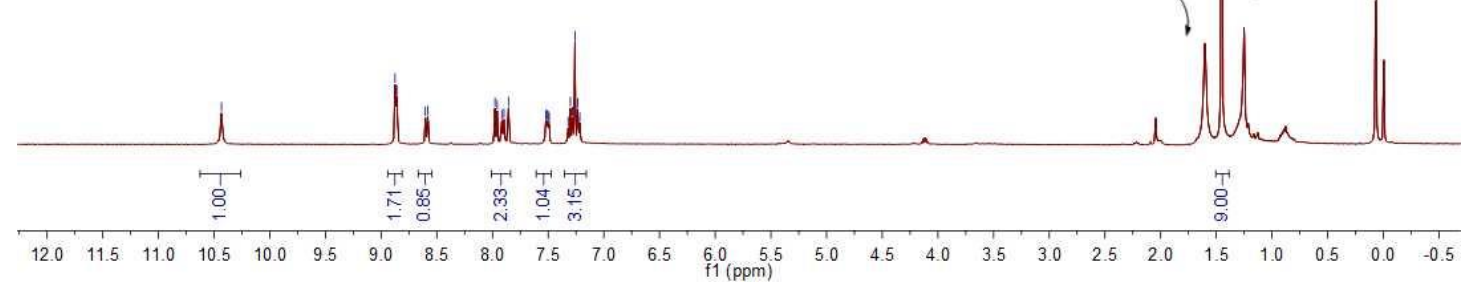

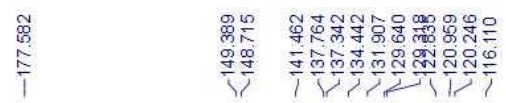
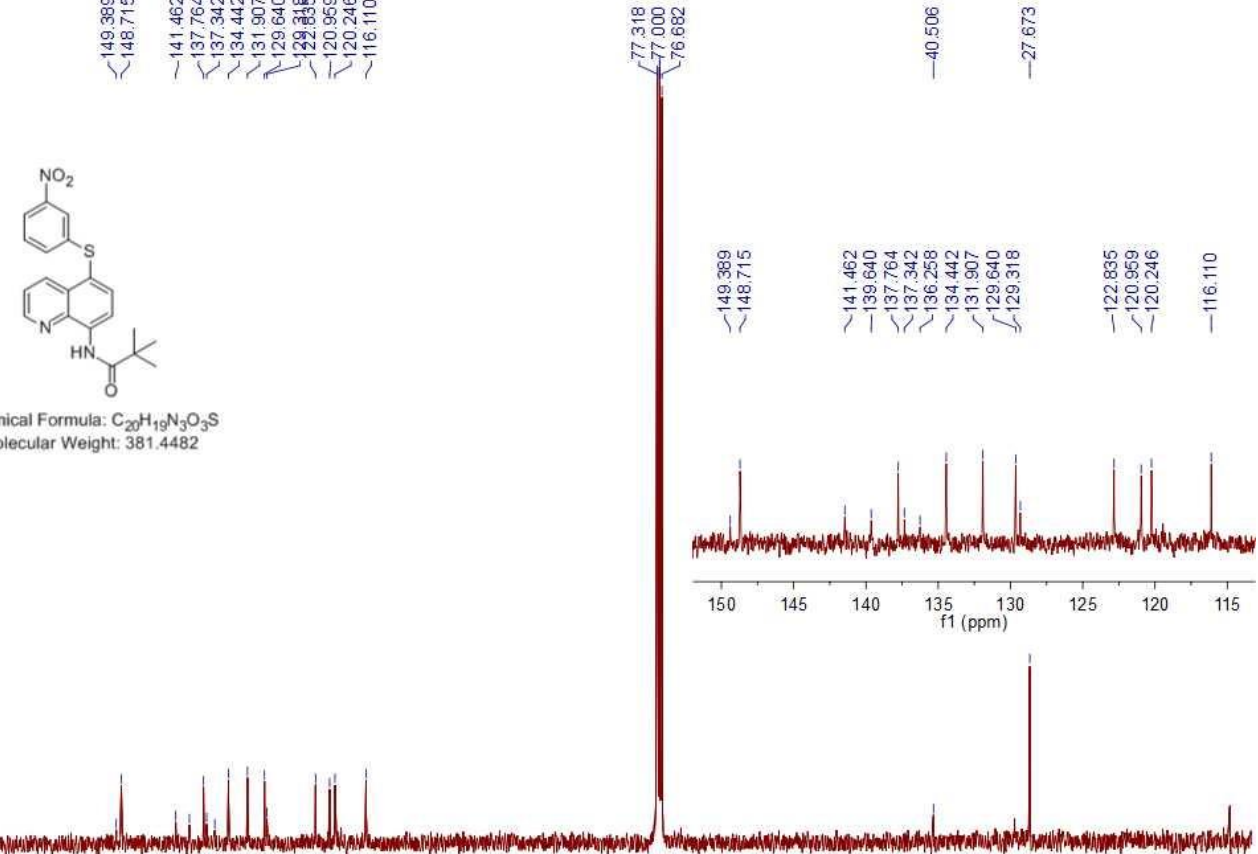

Chemical Formula: $\mathrm{C}_{20} \mathrm{H}_{19} \mathrm{~N}_{3} \mathrm{O}_{3} \mathrm{~S}$ Molecular Weight: 381.4482

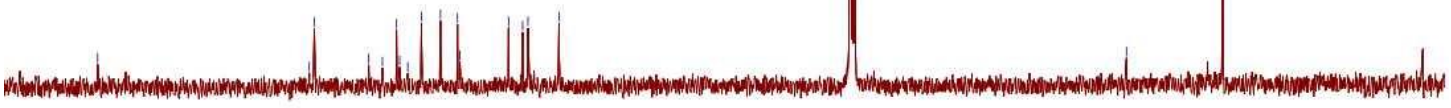

\begin{tabular}{llllllllllllllll}
\hline 0 & 180 & 170 & 160 & 150 & 140 & 130 & 120 & 110 & 100 & 90 & 1 & 1 & 1 & 1 & 1 \\
$\mathrm{f} 1(\mathrm{ppm})$ & 80 & 70 & 60 & 50 & 40 & 30 & 20 & 10 & 0
\end{tabular} 
$N$-(5-((2-nitrophenyl)thio)quinolin-8-yl)pivalamide (3h)
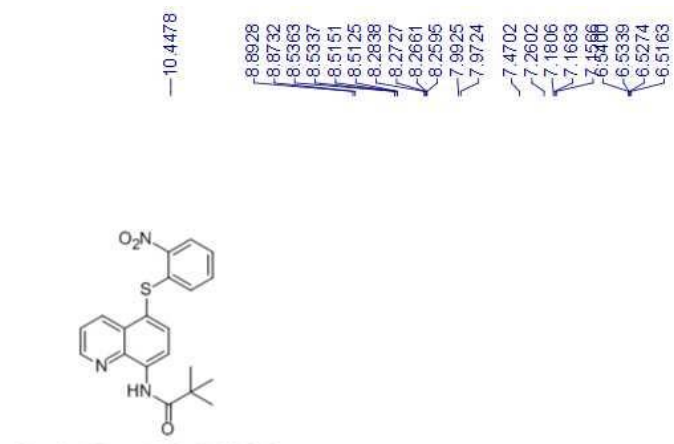

Chemical Formula: $\mathrm{C}_{20} \mathrm{H}_{19} \mathrm{~N}_{3} \mathrm{O}_{3} \mathrm{~S}$ Molecular Weight: 381,4482

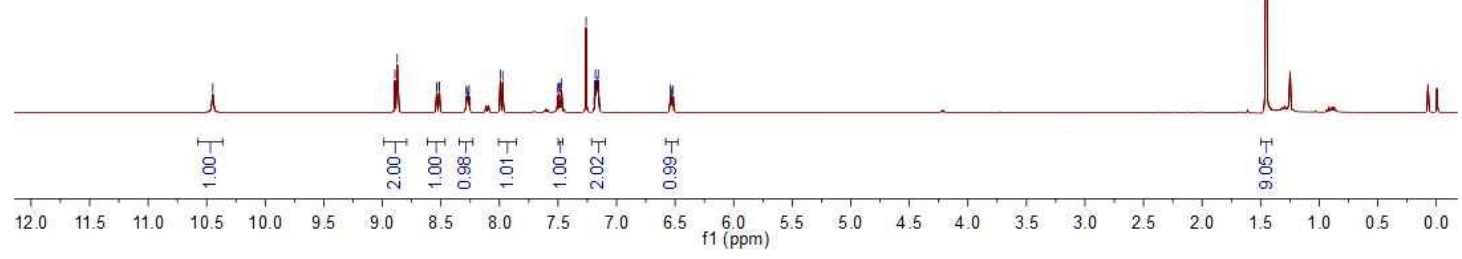

\&

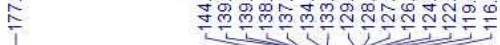

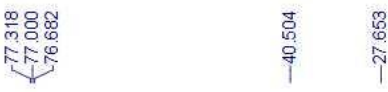

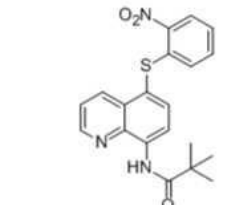

Chemical Formula: $\mathrm{C}_{20} \mathrm{H}_{19} \mathrm{~N}_{3} \mathrm{O}_{3} \mathrm{~S}$ Molecular Weight: 381.4482

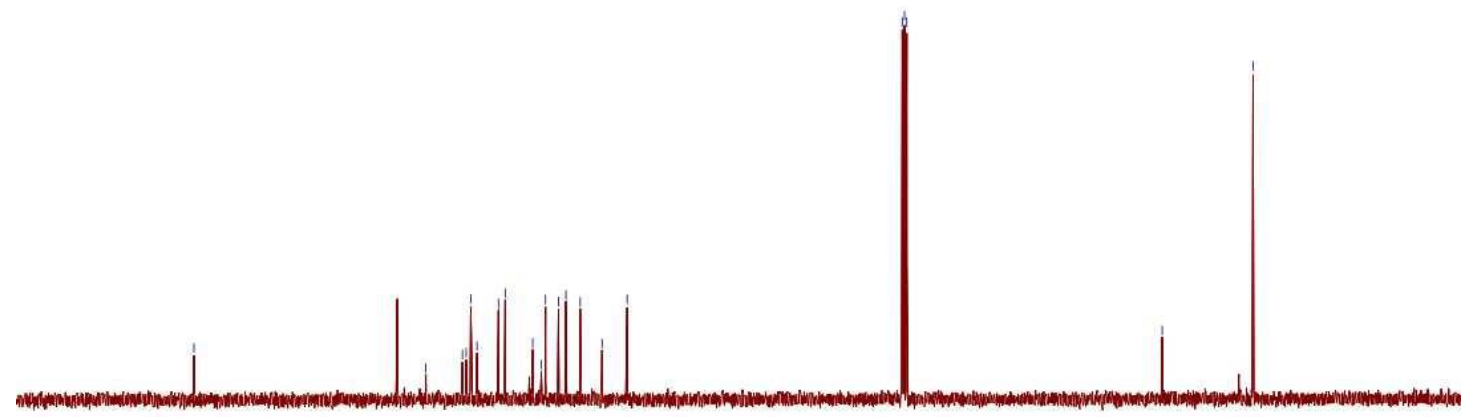

$30 \quad 120$

$110 \begin{gathered}100 \\ \mathrm{f} 1(\mathrm{ppm})\end{gathered}$

80

$60 \quad 50$

$30 \quad 20 \quad 10 \quad 0$ 
$N$-(5-((4-(tert-butyl)phenyl)thio)quinolin-8-yl)pivalamide (3i)
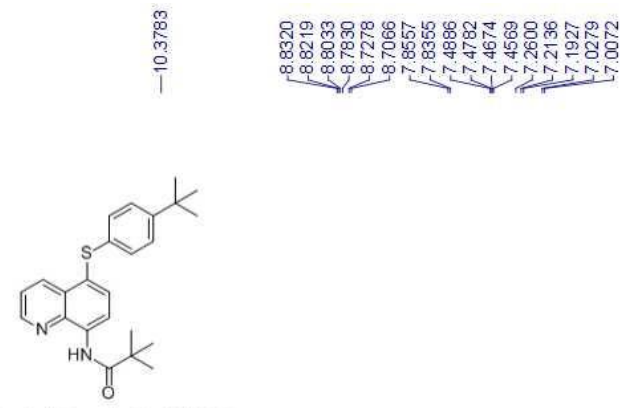

Chemical Formula: $\mathrm{C}_{24} \mathrm{H}_{28} \mathrm{~N}_{2} \mathrm{OS}$

Molecular Weight: 392.5569
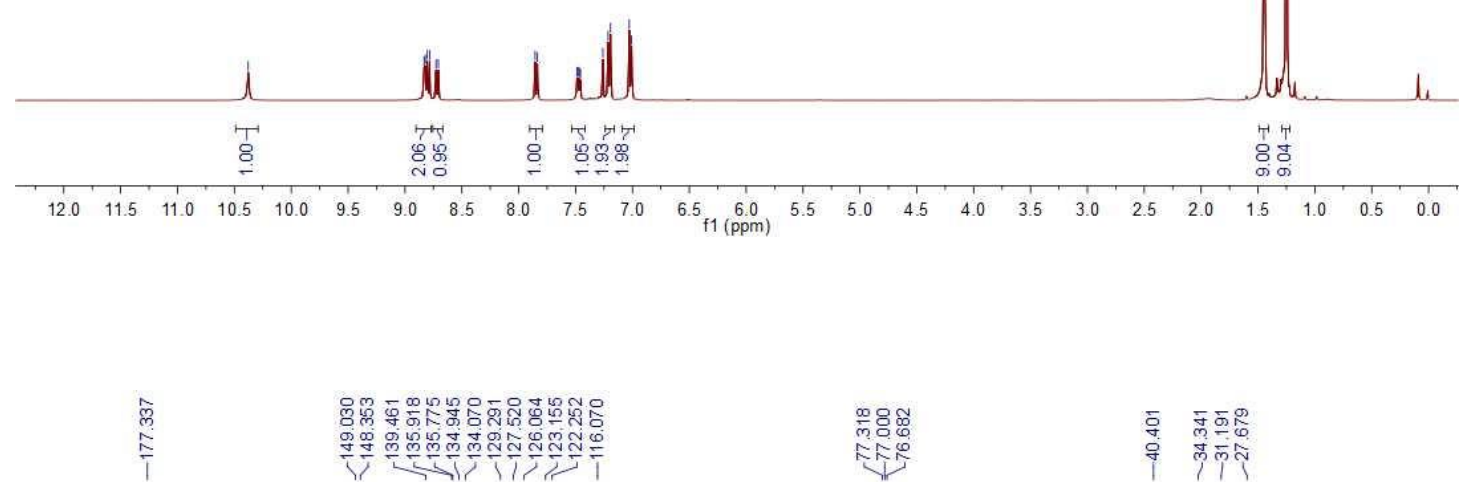

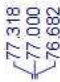

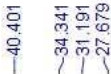

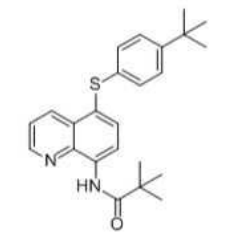

Chemical Formula: $\mathrm{C}_{24} \mathrm{H}_{28} \mathrm{~N}_{2} \mathrm{OS}$

Molecular Weight: 392.5569

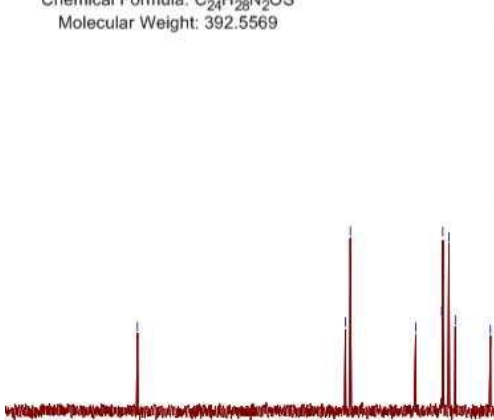

$\begin{array}{llllllllll}190 & 180 & 170 & 160 & 150 & 140 & 130 & 120 & 110 & \begin{array}{l}100 \\ \mathrm{f} 1(\mathrm{ppm})\end{array}\end{array}$

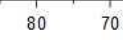

$60 \quad 50$

$\begin{array}{lllll}40 & 30 & 20 & 10 & 1\end{array}$ 
$N$-(5-(cyclohexylthio)quinolin-8-yl)pivalamide (3j)

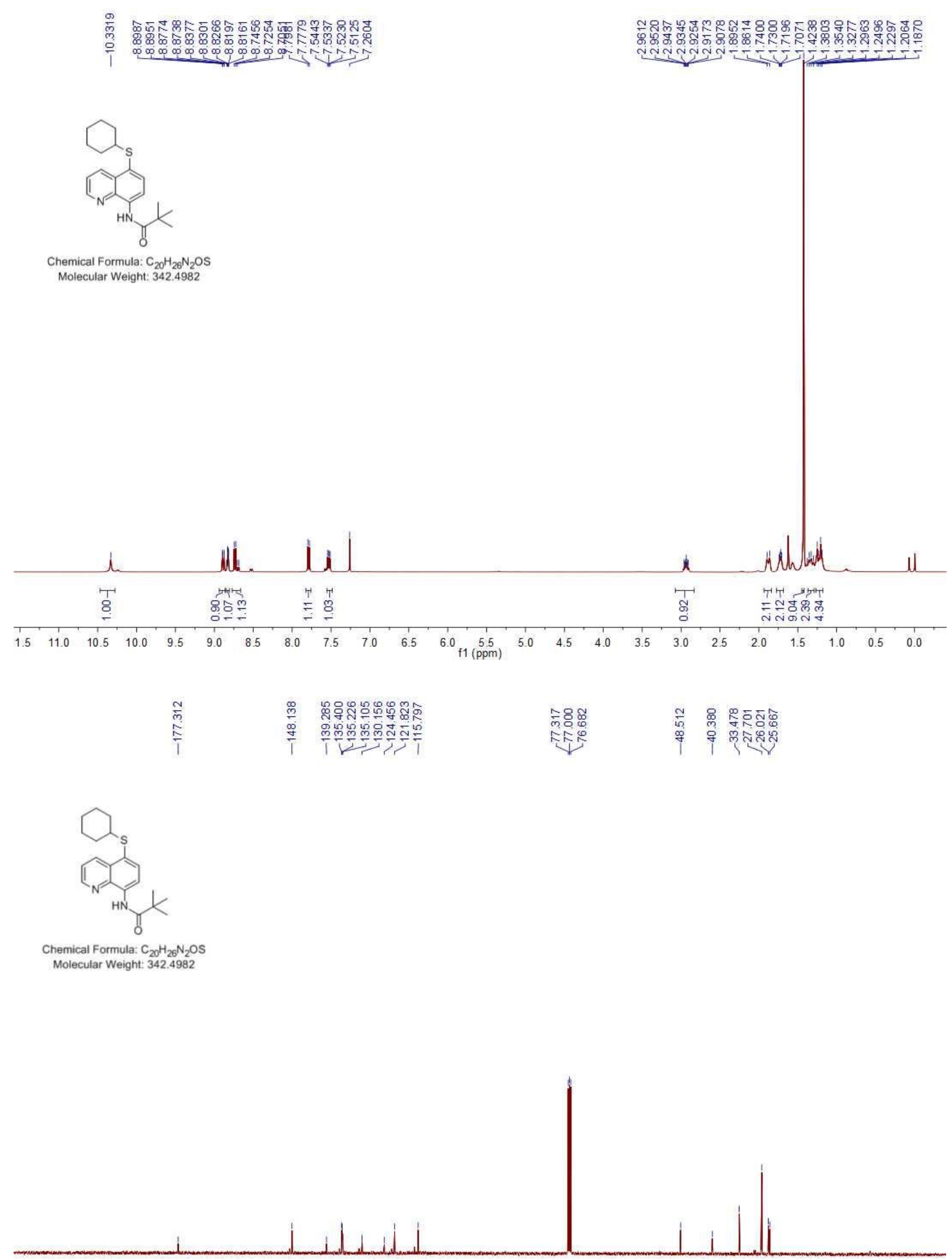

$\begin{array}{lllllllllllllllllllllllll}210 & 200 & 190 & 180 & 170 & 160 & 150 & 140 & 130 & 120 & 110 & 100 & 90 & 80 & 70 & 60 & 50 & 40 & 30 & 20 & 10 & 0 & -10\end{array}$ 
$N$-(5-(propylthio)quinolin-8-yl)pivalamide (3k)

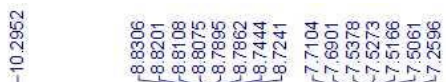

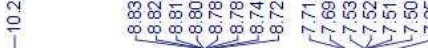

等

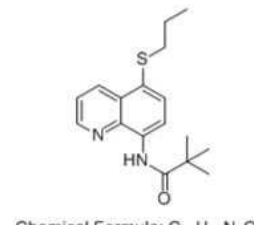

Chemical Formula: $\mathrm{C}_{17} \mathrm{H}_{22} \mathrm{~N}_{2} \mathrm{OS}$

Molecular Weight: 302.4344

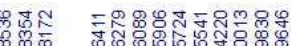

ฟกำ

낭
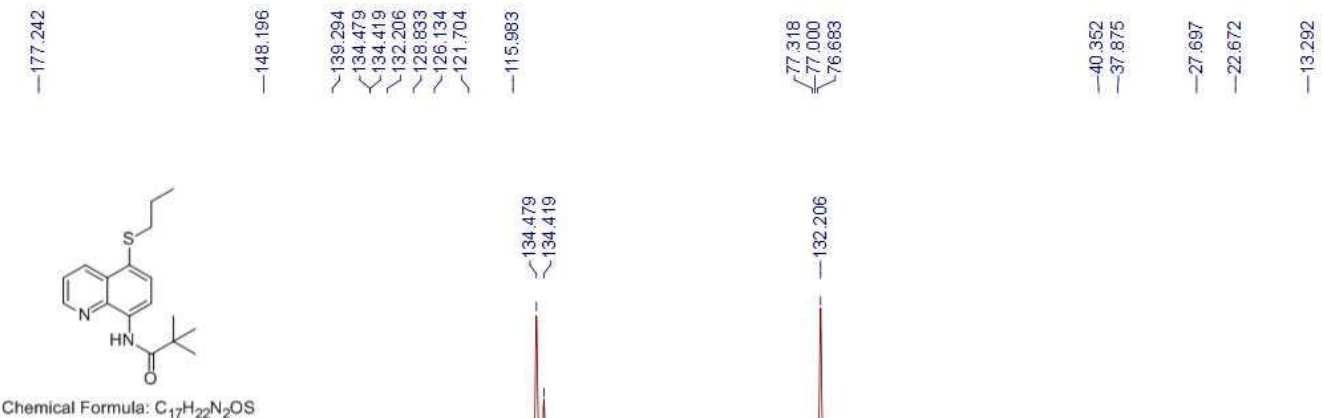

Chemical Formula: $\mathrm{C}_{17} \mathrm{H}_{22} \mathrm{~N}_{2} \mathrm{O}$

Molecular Weight: 302.4344
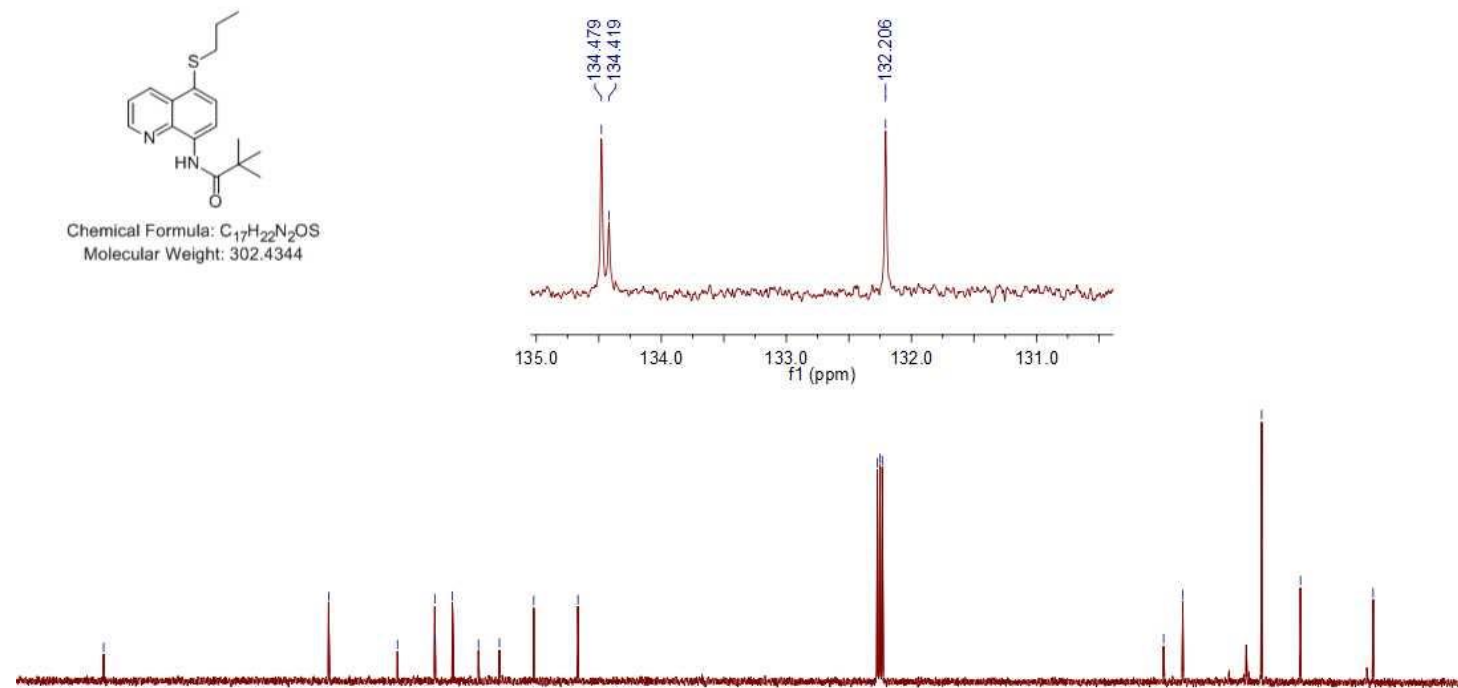

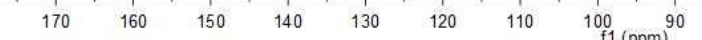

$80 \quad 70 \quad 60$

50
wh

त्र 
$N$-(5-(phenylthio)quinolin-8-yl)acetamide (Aa)

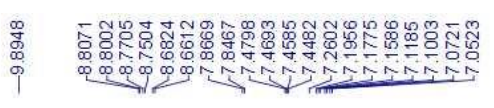

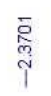

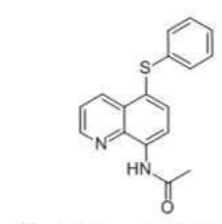

Chemical Formula: $\mathrm{C}_{17} \mathrm{H}_{14} \mathrm{~N}_{2} \mathrm{OS}$
Molecular Weight: 294.3709
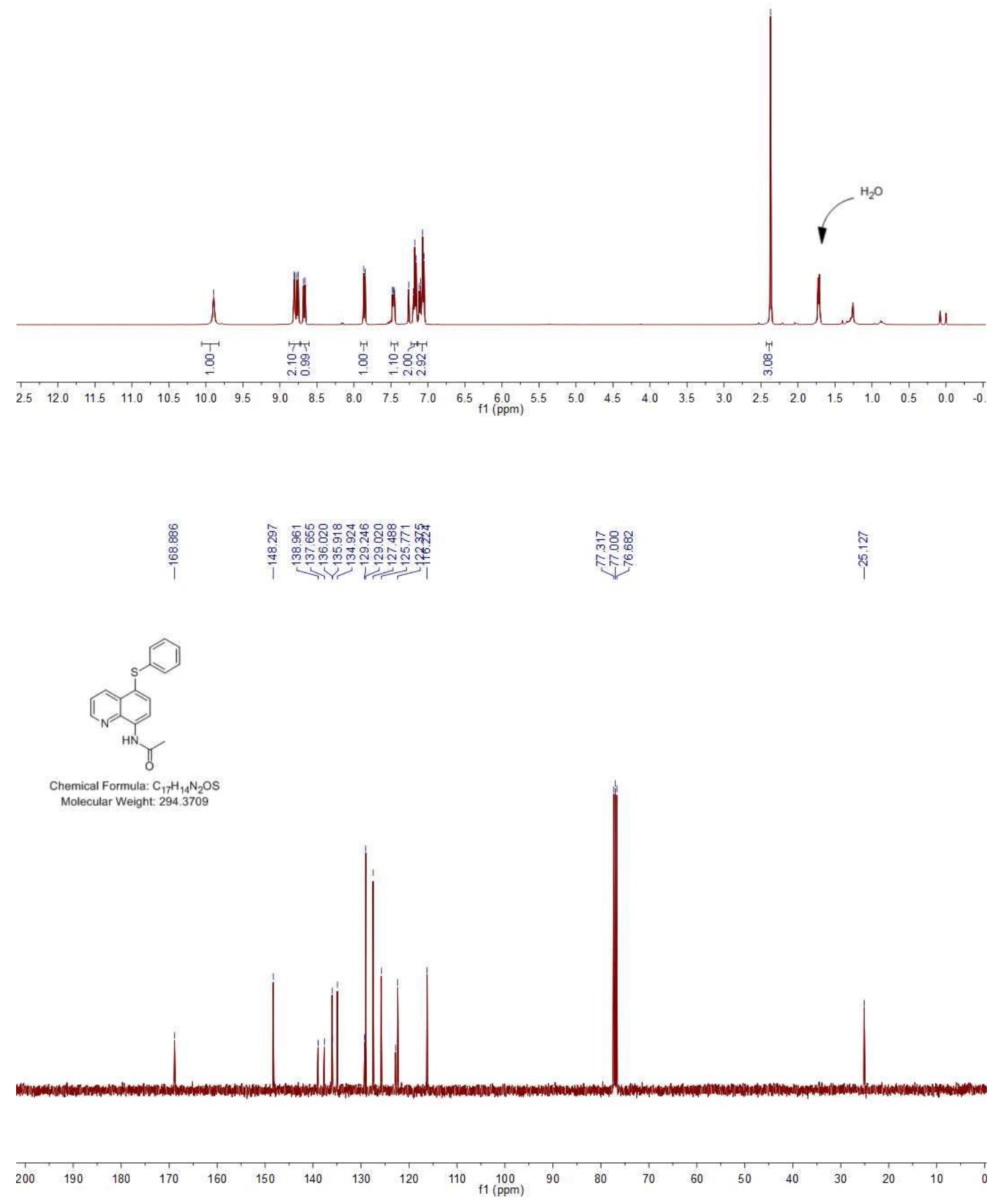

S51 
$N$-(5-(p-tolylthio)quinolin-8-yl)acetamide (4b)
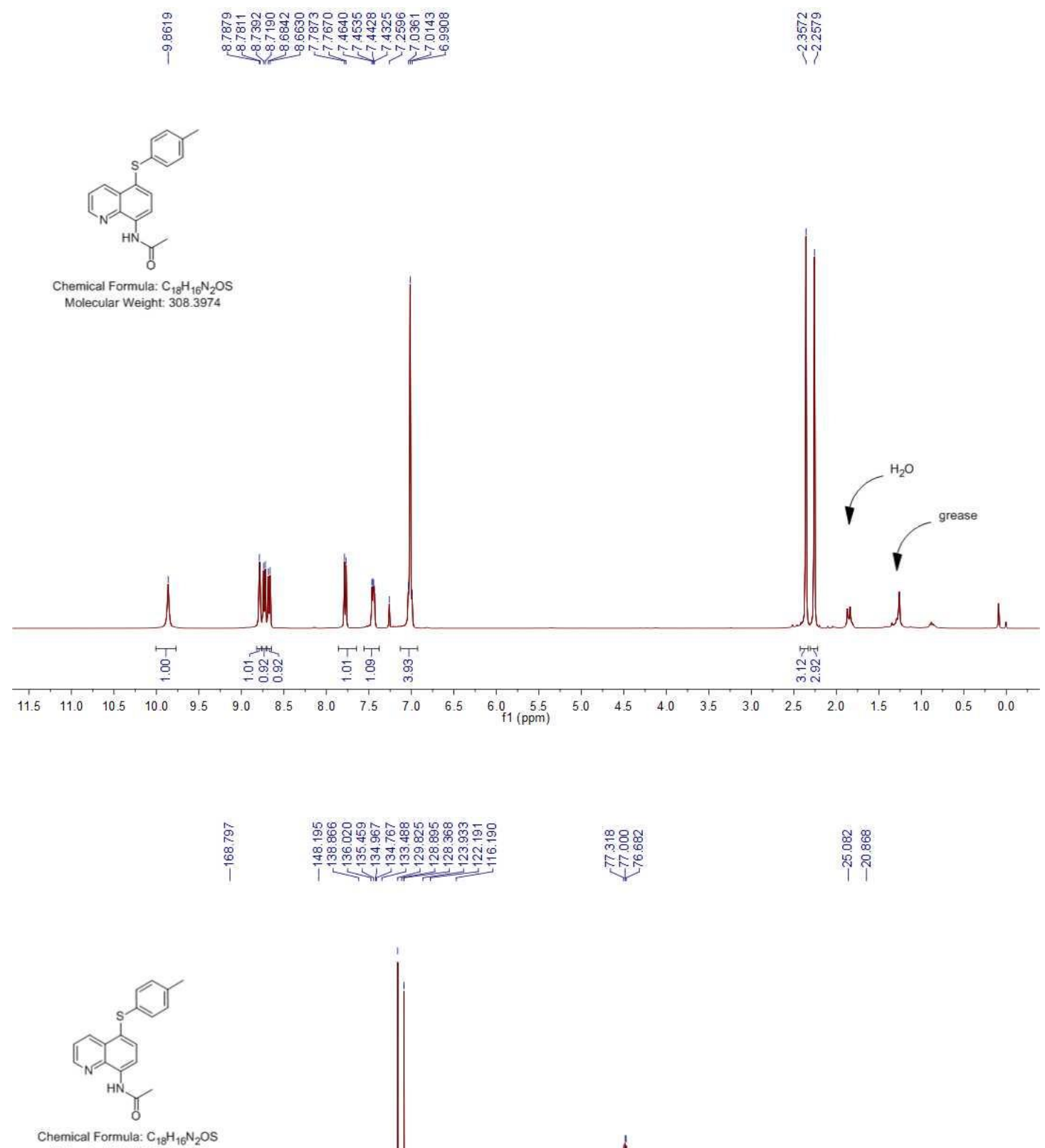

Molection $\mathrm{C}_{18} \mathrm{H}_{16} \mathrm{~N}_{2} \mathrm{OS}$

Molecular Weight: 308.3974

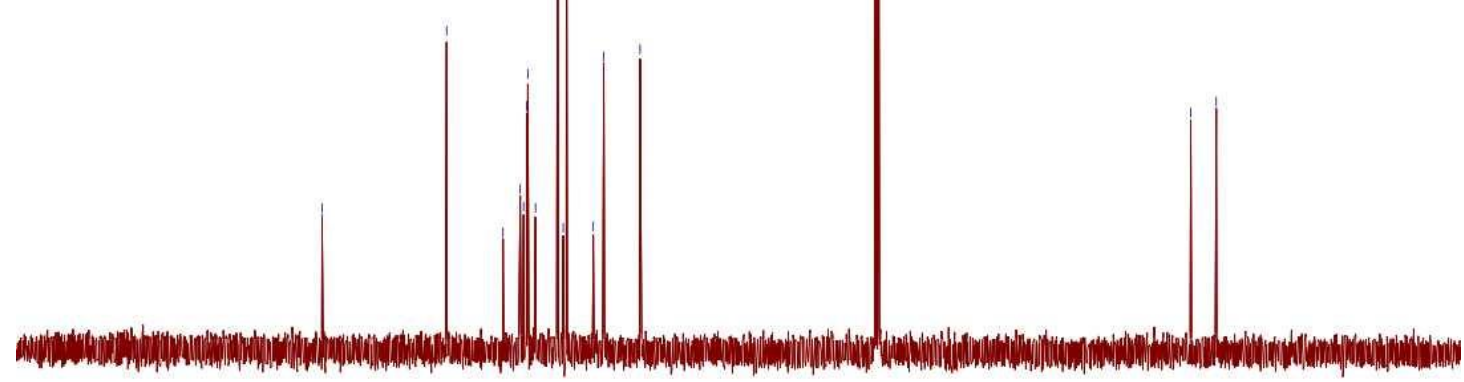

$\begin{array}{lllllllllllllllllllllll}210 & 200 & 190 & 180 & 170 & 160 & 150 & 140 & 130 & 120 & 110 & 100 & 90 & 80 & 70 & 60 & 50 & 40 & 30 & 20 & 10 & 0 & -10\end{array}$ 
$N$-(5-((4-methoxyphenyl)thio)quinolin-8-yl)acetamide (4c)
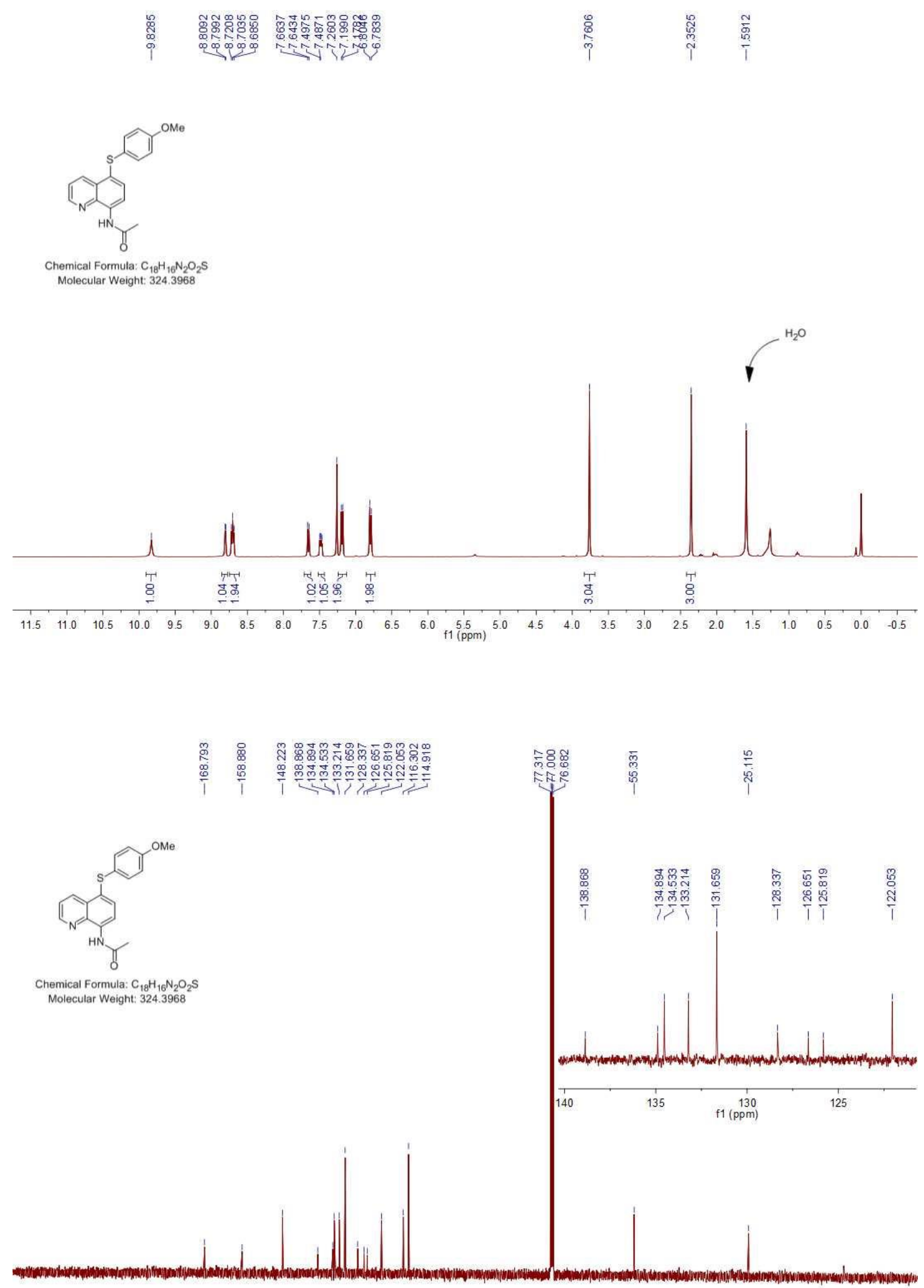

$\begin{array}{lllllllllllllllllllllll}210 & 200 & 190 & 180 & 170 & 160 & 150 & 140 & 130 & 120 & 110 & 100 & 90 & 80 & 70 & 60 & 50 & 40 & 30 & 20 & 10 & 0 & -10\end{array}$ 
$N$-(5-((4-chlorophenyl)thio)quinolin-8-yl)acetamide (4d)
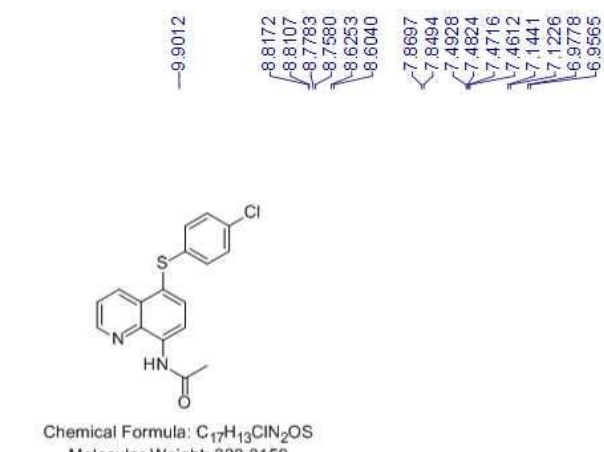

Molecular Weight: 328.8159

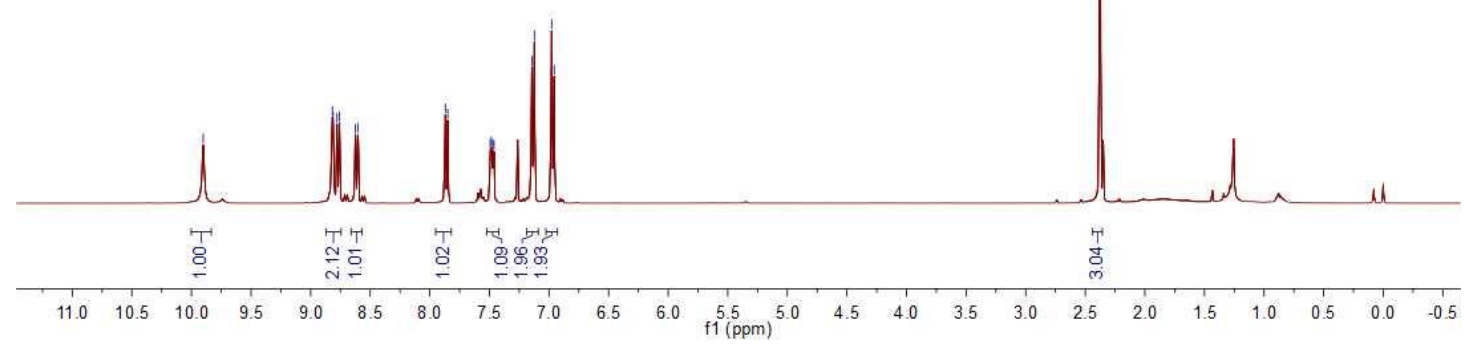

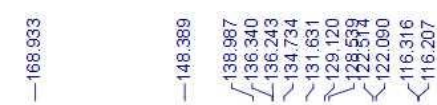

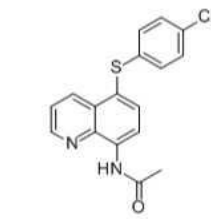

Chemical Formula: $\mathrm{C}_{17} \mathrm{H}_{13} \mathrm{CIN}_{2} \mathrm{OS}$ Molecular Weight: 328.8159
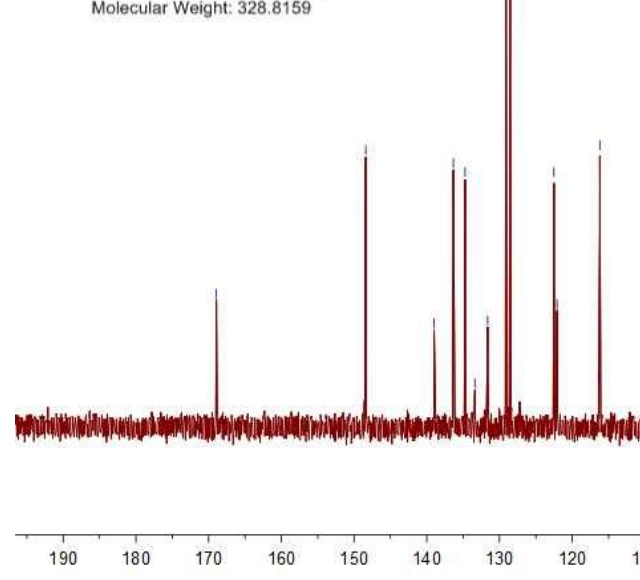

$140 \quad 130$

12

110

$1(\mathrm{ppm}) 90$ 
2,2-dimethyl- $N$-(5-(phenylthio)quinolin-8-yl)butanamide (4e)

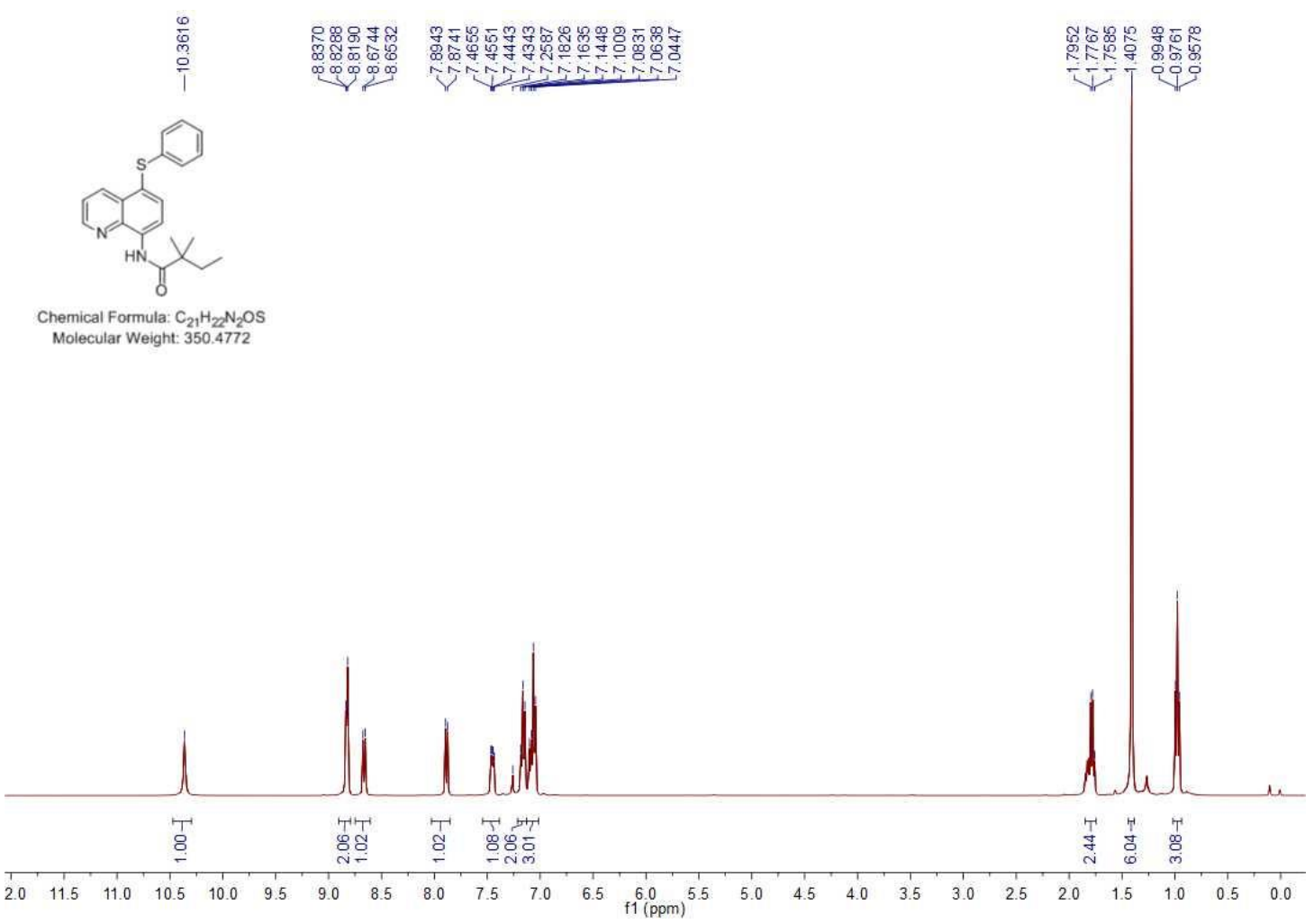

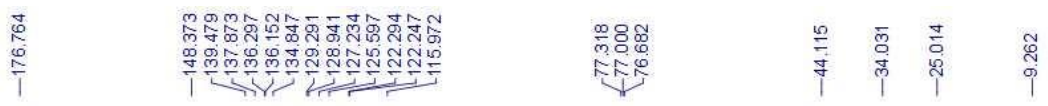

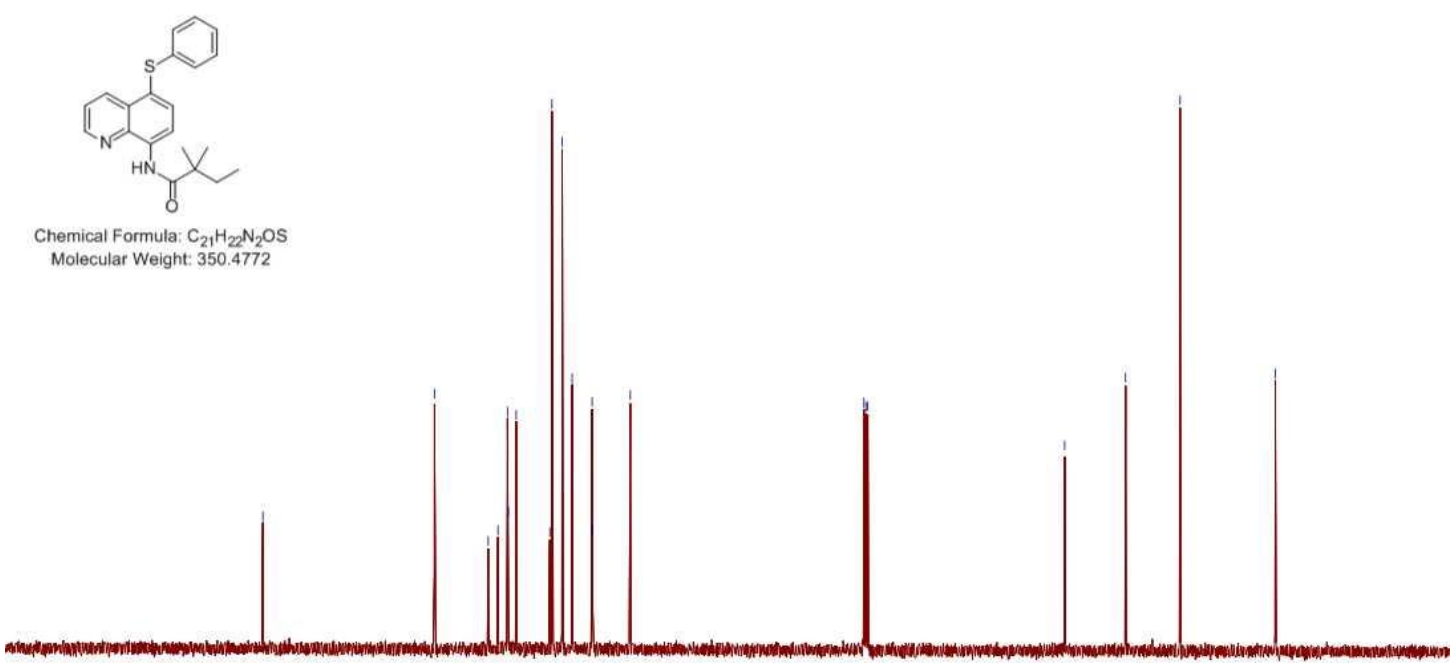

$\begin{array}{lllllllllllllllllllllllllllll}210 & 200 & 190 & 180 & 170 & 160 & 150 & 140 & 130 & 120 & 110 & \begin{array}{c}100 \\ \mathrm{f} 1(\mathrm{ppm})\end{array} & 90 & 80 & 70 & 60 & 50 & 40 & 30 & 20 & 10 & 0 & -10\end{array}$ 

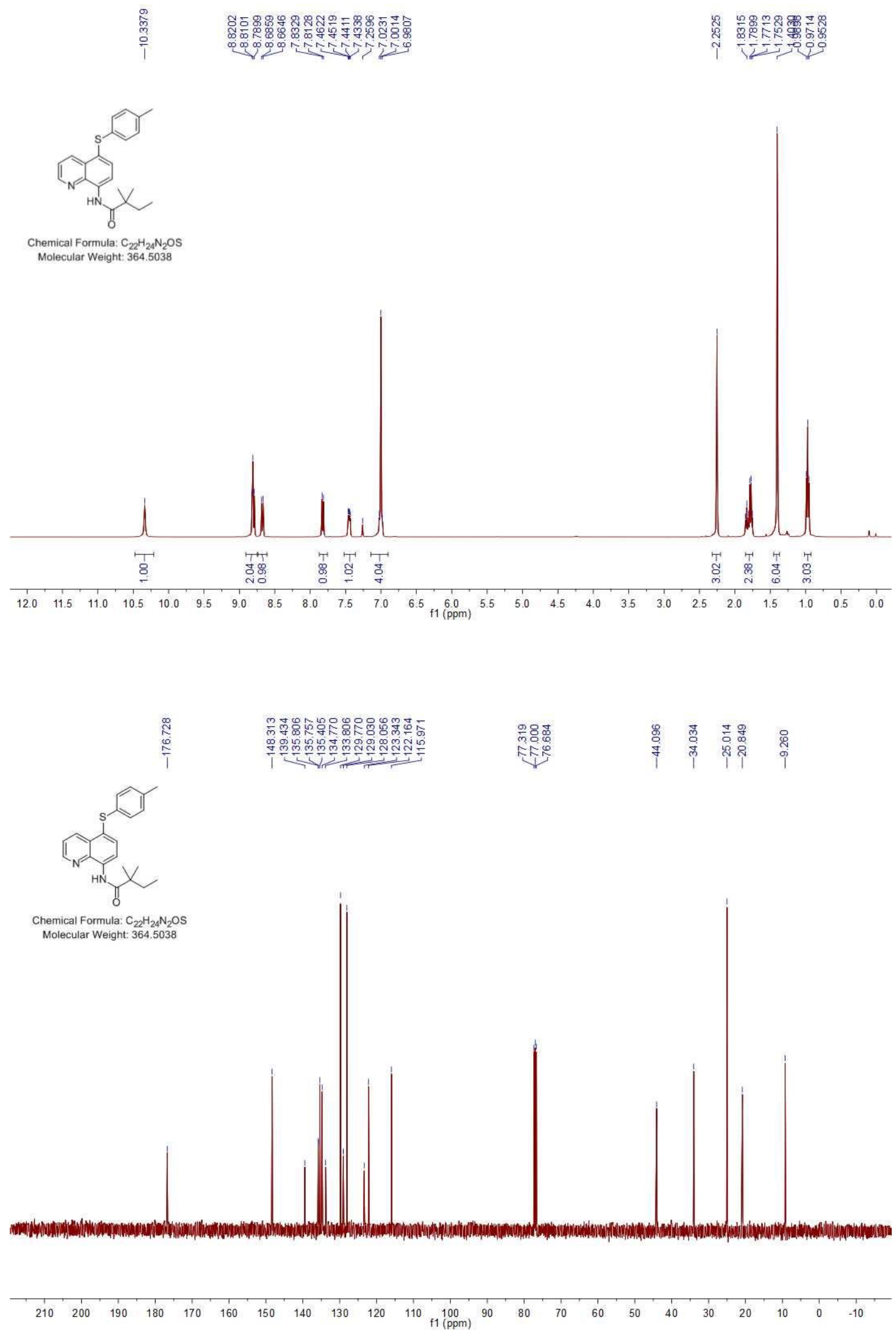
$N$-(5-((4-methoxyphenyl)thio)quinolin-8-yl)-2,2-dimethylbutanamide (4g)
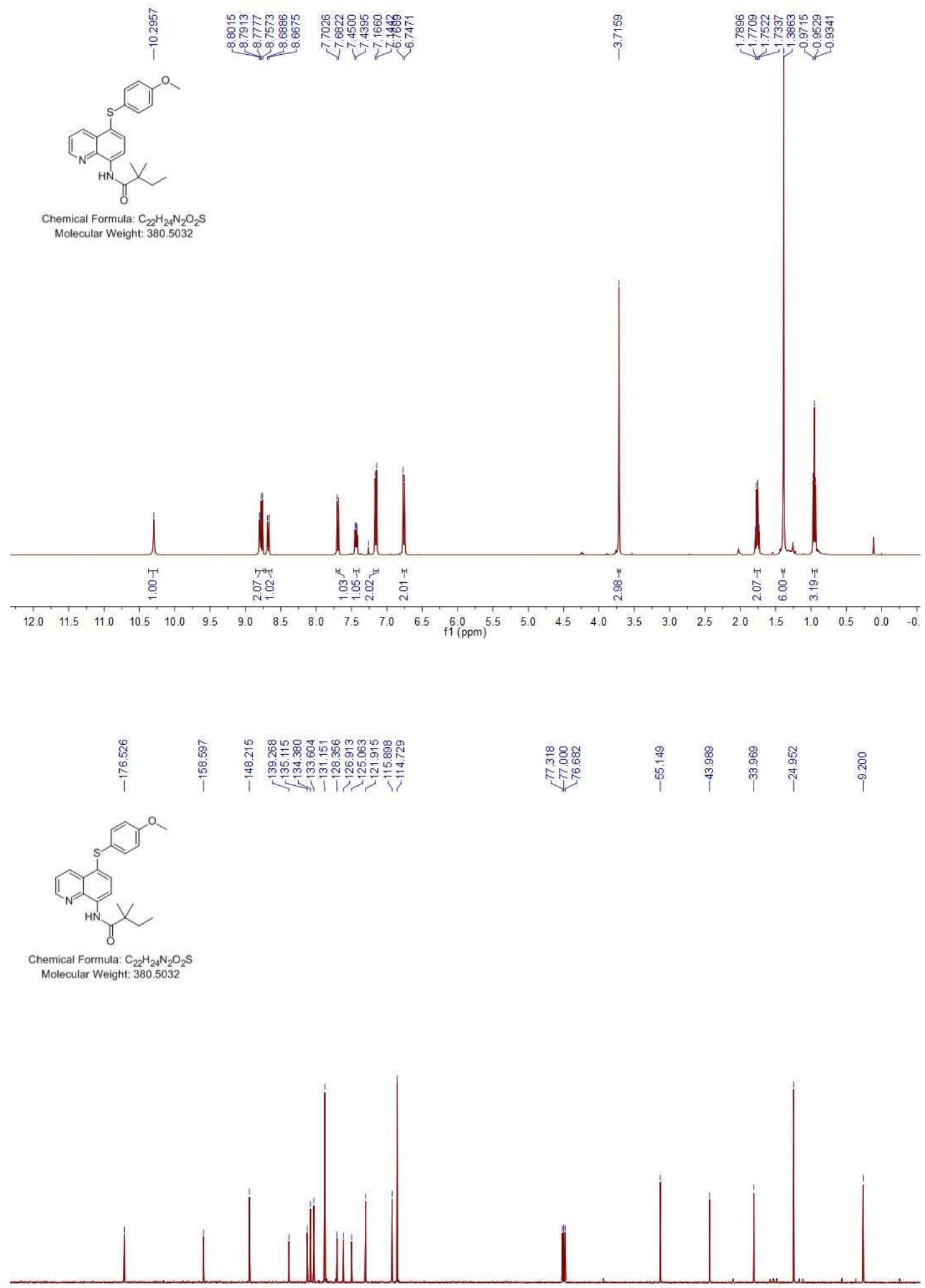

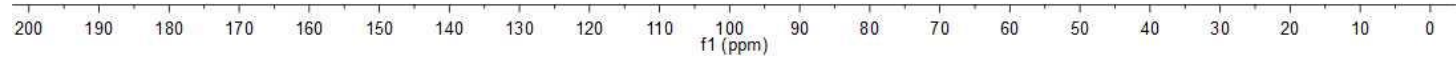


$N$-(5-((4-chlorophenyl)thio)quinolin-8-yl)-2,2-dimethylbutanamide (4h)
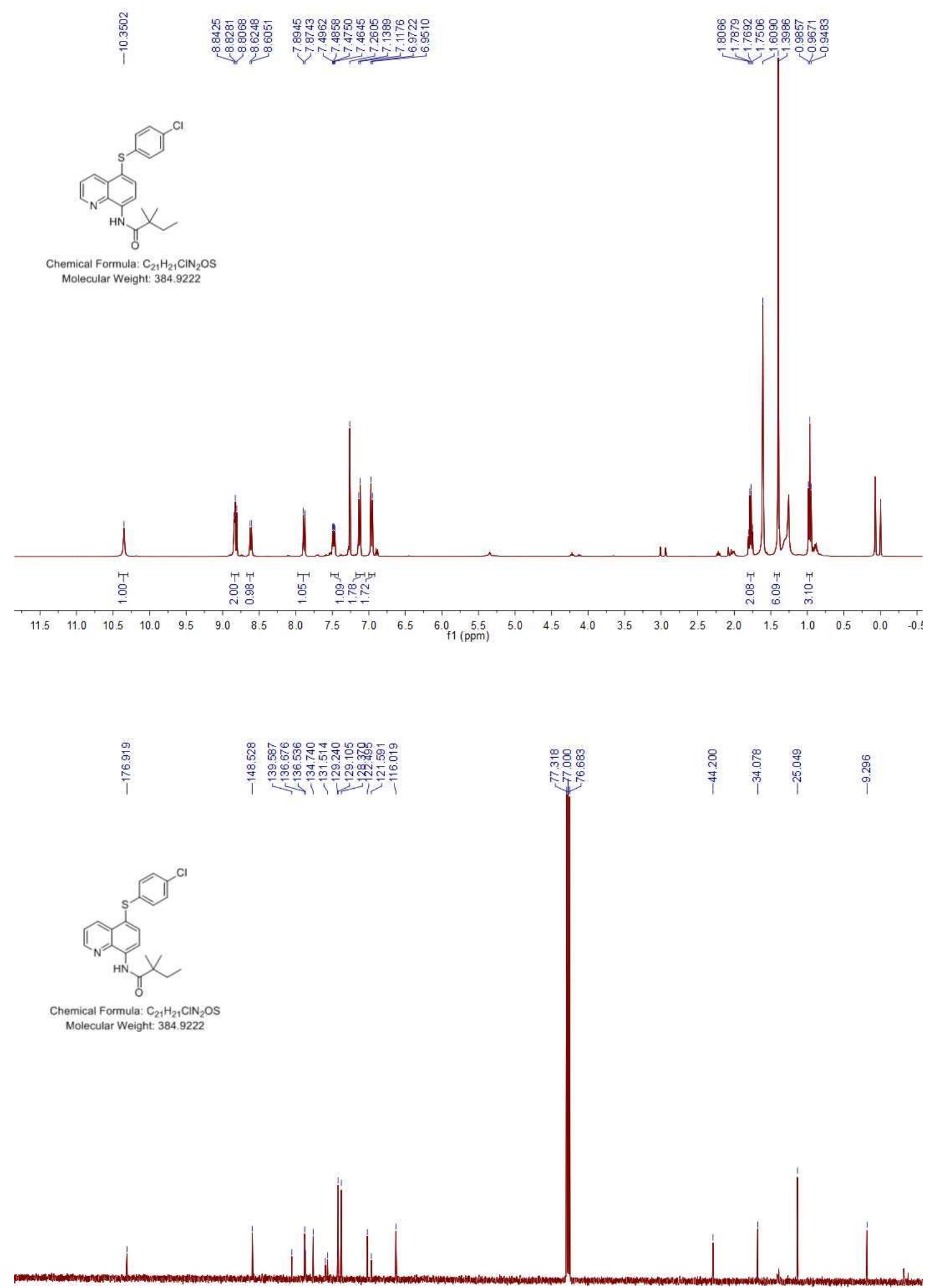

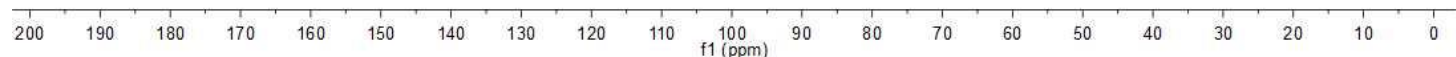


2-ethyl- $N$-(5-(phenylthio)quinolin-8-yl)butanamide (4i)

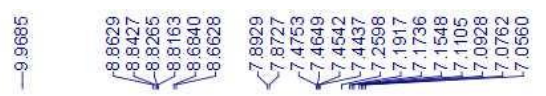

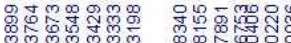

NNNNNNA

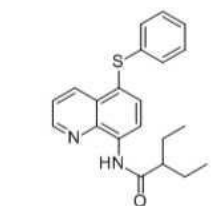

Chemical Formula: $\mathrm{C}_{21} \mathrm{H}_{22} \mathrm{~N}_{2} \mathrm{OS}$

Molecular Weight: 350.4772
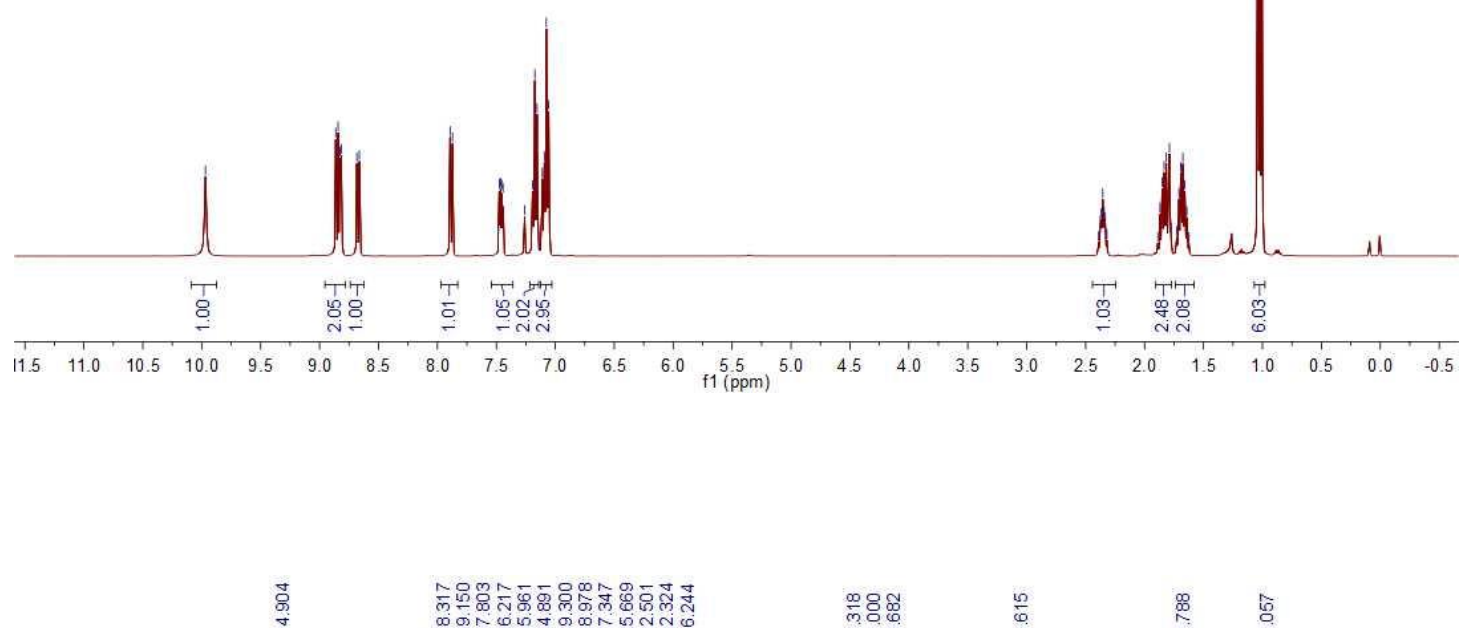

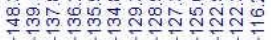

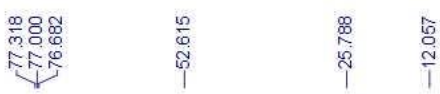

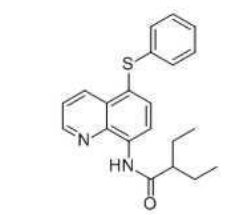

Chemical Formula: $\mathrm{C}_{21} \mathrm{H}_{22} \mathrm{~N}_{2} \mathrm{OS}$ Molecular Weight: 350.4772

$\begin{array}{llllllllllllllllllllllllllll}210 & 200 & 190 & 180 & 170 & 160 & 150 & 140 & 130 & 120 & 110 & \begin{array}{c}100 \\ \mathrm{f} 1(\mathrm{ppm})\end{array} & 90 & 80 & 70 & 60 & 50 & 40 & 30 & 20 & 10 & 0 & -10\end{array}$ 
$N$-(5-(phenylthio)quinolin-8-yl)cyclopentanecarboxamide (4j)
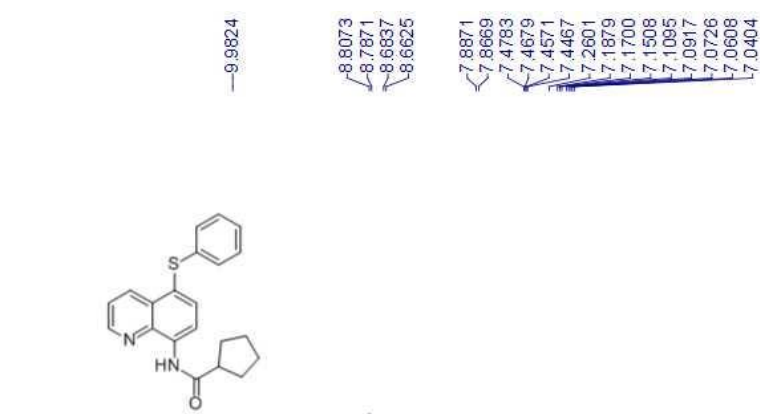

Chemical Formula: $\mathrm{C}_{21} \mathrm{H}_{20} \mathrm{~N}_{2} \mathrm{OS}$ Molecular Weight: 348.4613
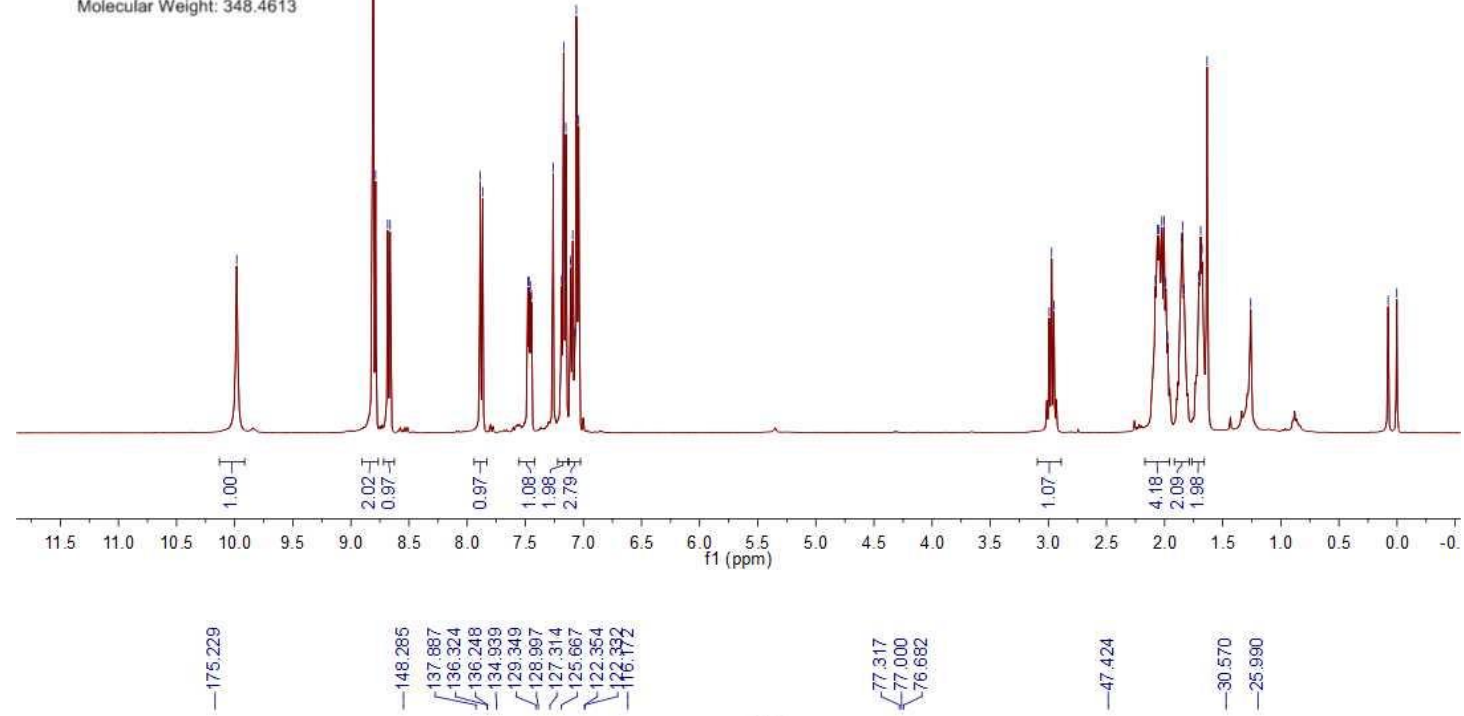

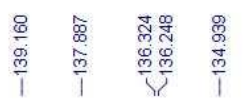

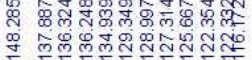
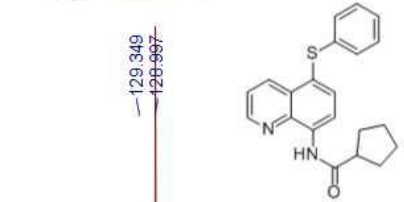

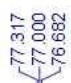

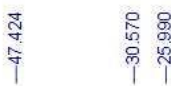

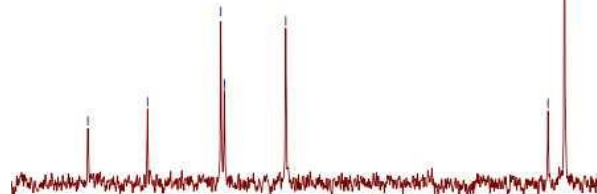

Chemical Formula: $\mathrm{C}_{2}, \mathrm{H}_{20} \mathrm{~N}_{2} \mathrm{OS}$ Molecular Weight: 348.4613

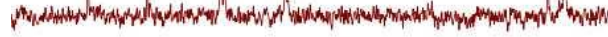

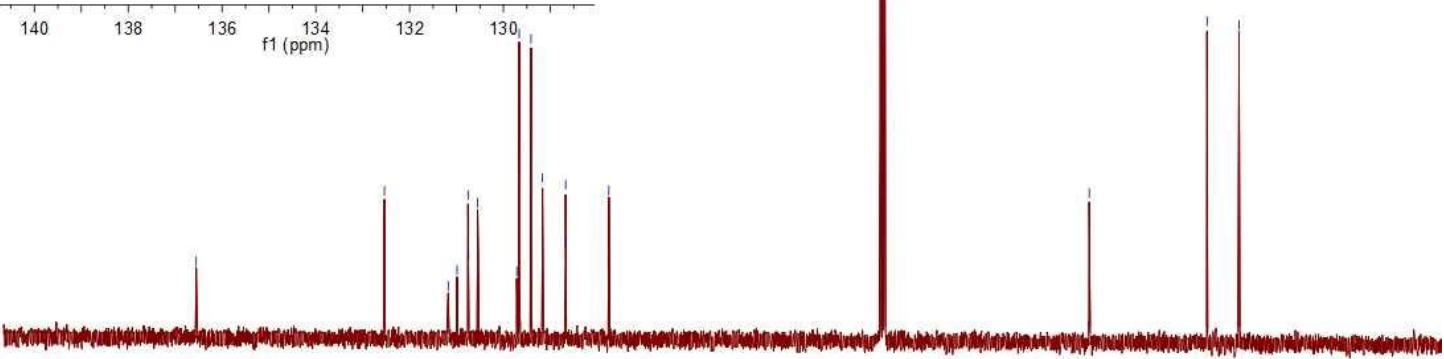

200

190

$180 \quad 170$

150

$110 \quad 100$

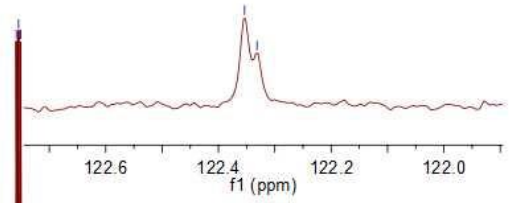


$N$-(5-(p-tolylthio)quinolin-8-yl)cyclopentanecarboxamide (4k)

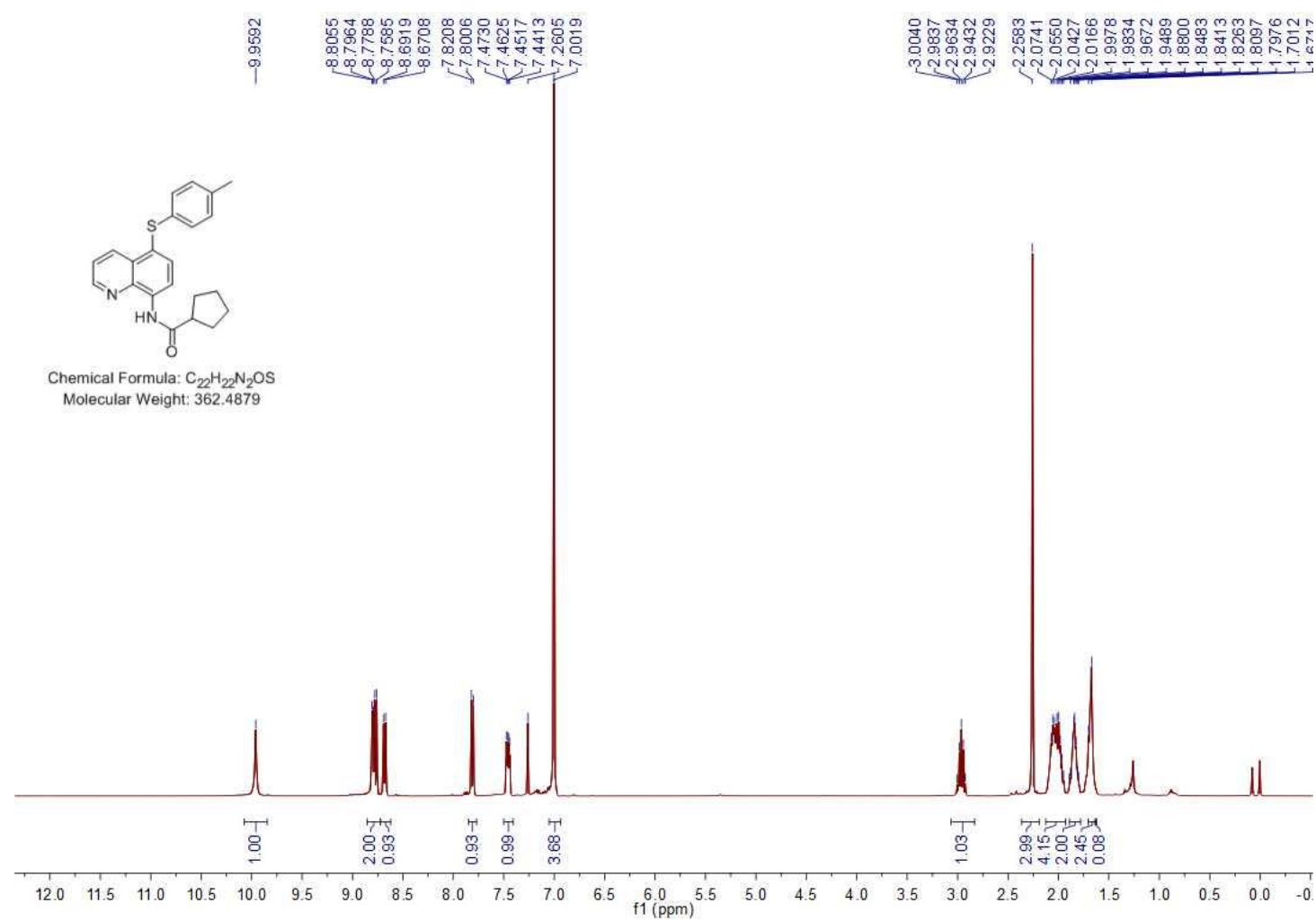

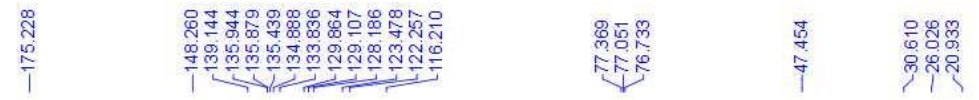

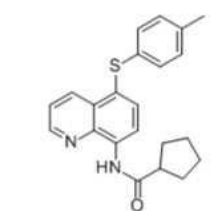

Chemical Formula: $\mathrm{C}_{22}$ Molecular Weight: 362.4879

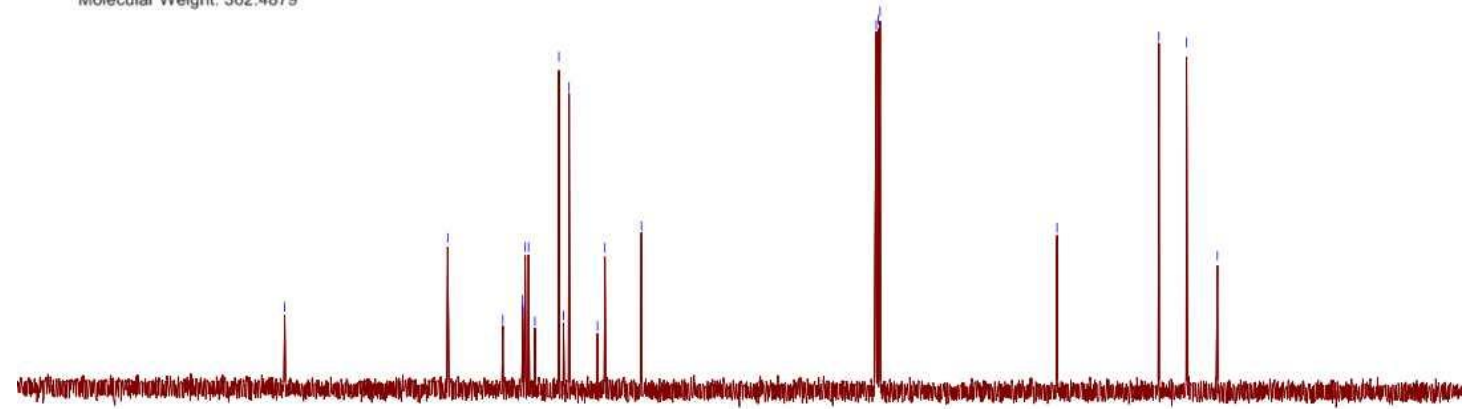

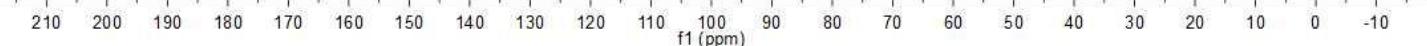


$N$-(5-((4-methoxyphenyl)thio)quinolin-8-yl)cyclopentanecarboxamide (41)

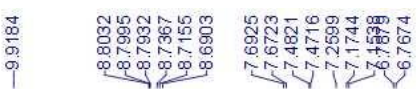

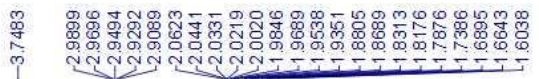

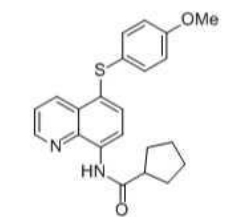

Chemical Formula: $\mathrm{C}_{22} \mathrm{H}_{22} \mathrm{~N}_{2} \mathrm{O}_{2}$ Molecular Weight: 378.4873
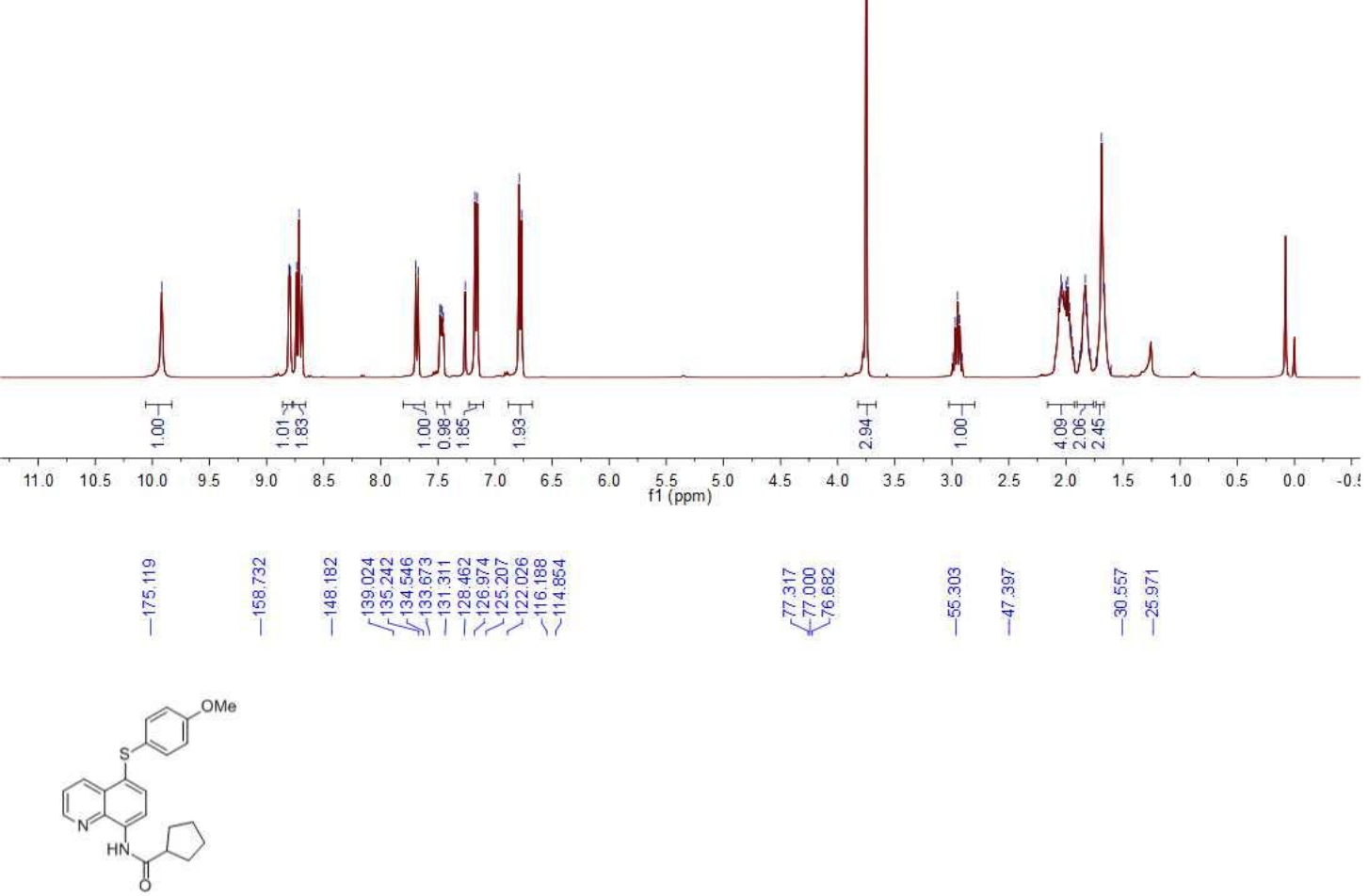

Chemical Formula: $\mathrm{C}_{2} \mathrm{H}_{22} \mathrm{~N}_{2} \mathrm{O}_{2} \mathrm{~S}$

Molecular Weight: 378.4873

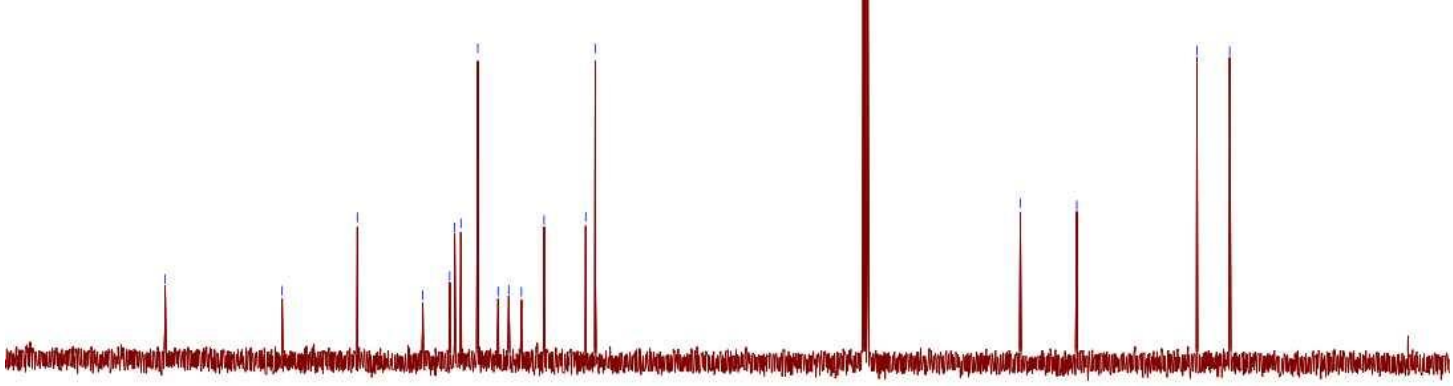

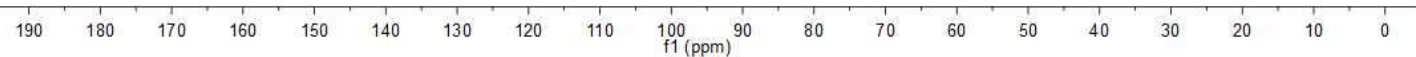


$N$-(5-((4-chlorophenyl)thio)quinolin-8-yl)cyclopentanecarboxamide (4m)
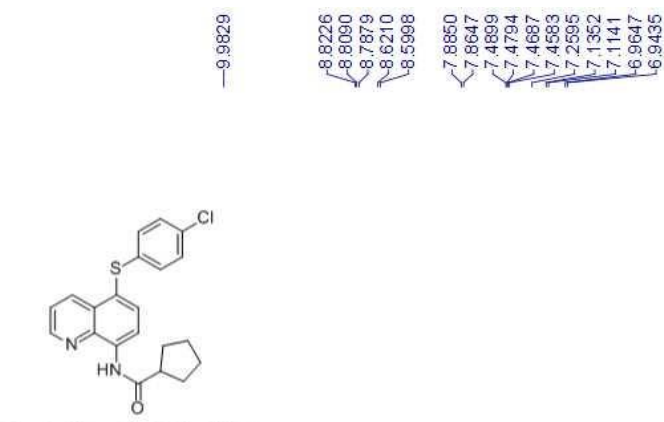

Chemical Formula: $\mathrm{C}_{21} \mathrm{H}_{19} \mathrm{CIN}_{2} \mathrm{OS}$ Molecular Weight: 382.9064

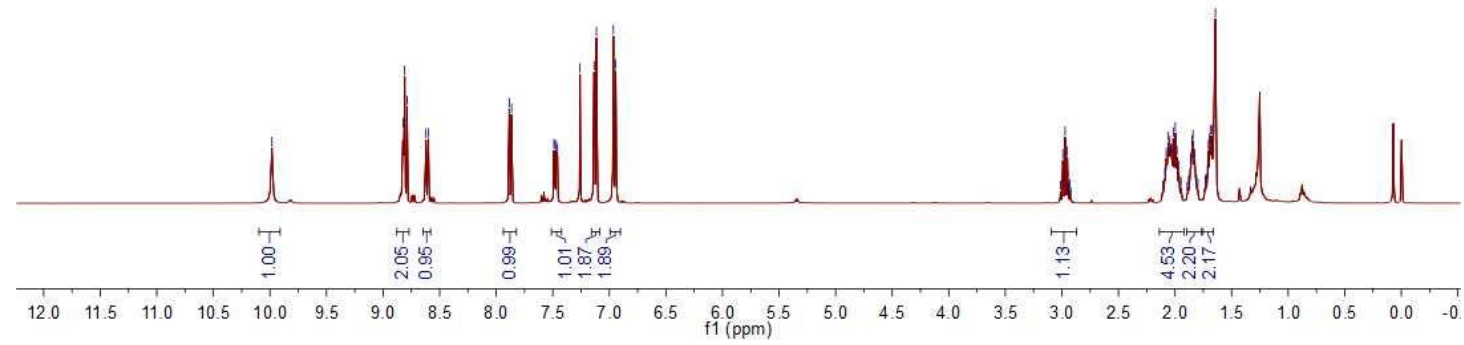

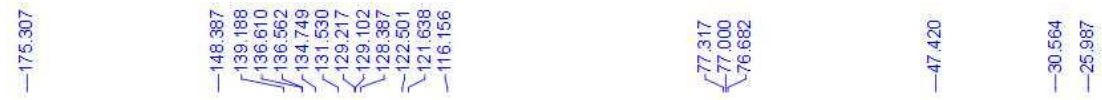

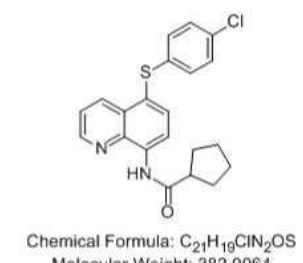

Molecular Weight: 382.9064

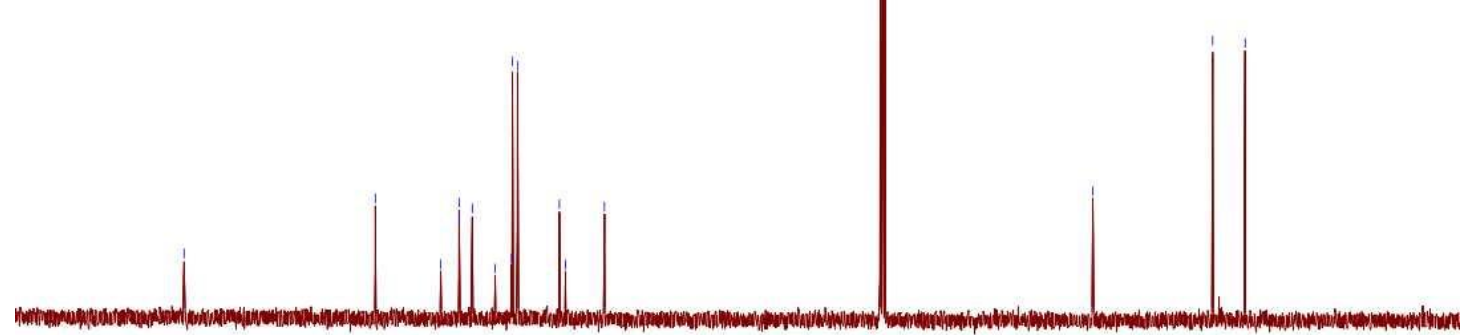

190

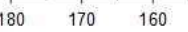

$150 \quad 140$

$\begin{array}{lll}120 & 110 & 100 \\ \mathrm{f} 1(\mathrm{ppm})\end{array} 90$

80

60

50

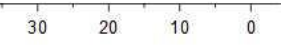


1-methyl- $N$-(5-(phenylthio)quinolin-8-yl)cyclohexanecarboxamide (4n)
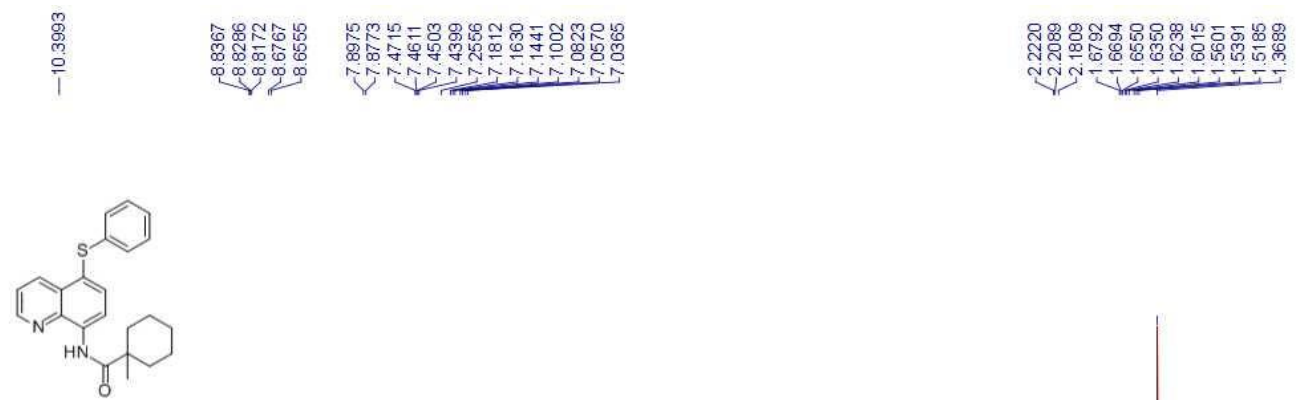

Chemical Formula: $\mathrm{C}_{23} \mathrm{H}_{24} \mathrm{~N}_{2} \mathrm{OS}$

Molecular Weight: 376.5145
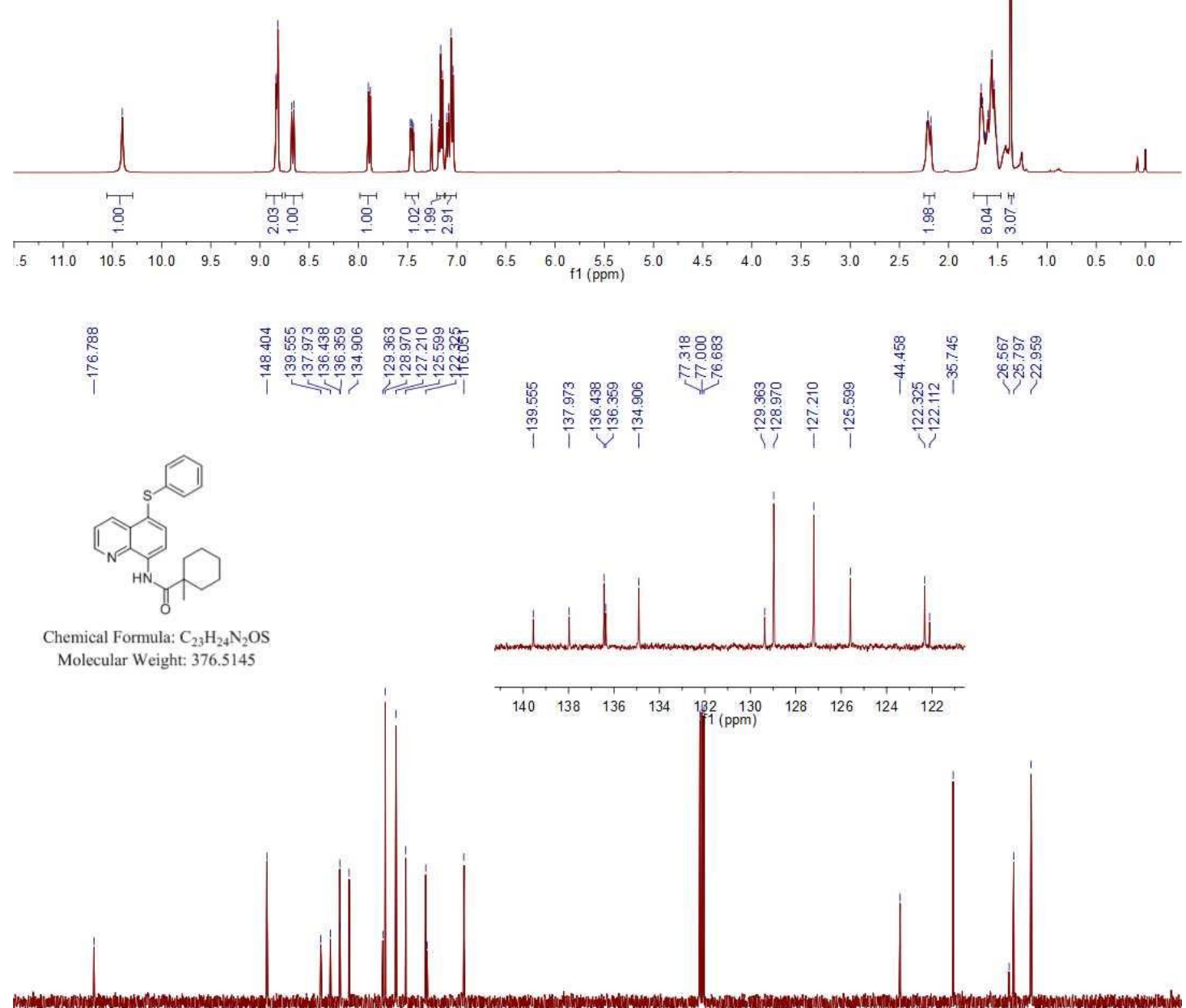

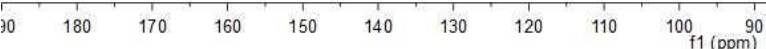


$N$-(5-(phenylthio)quinolin-8-yl)octanamide (4o)
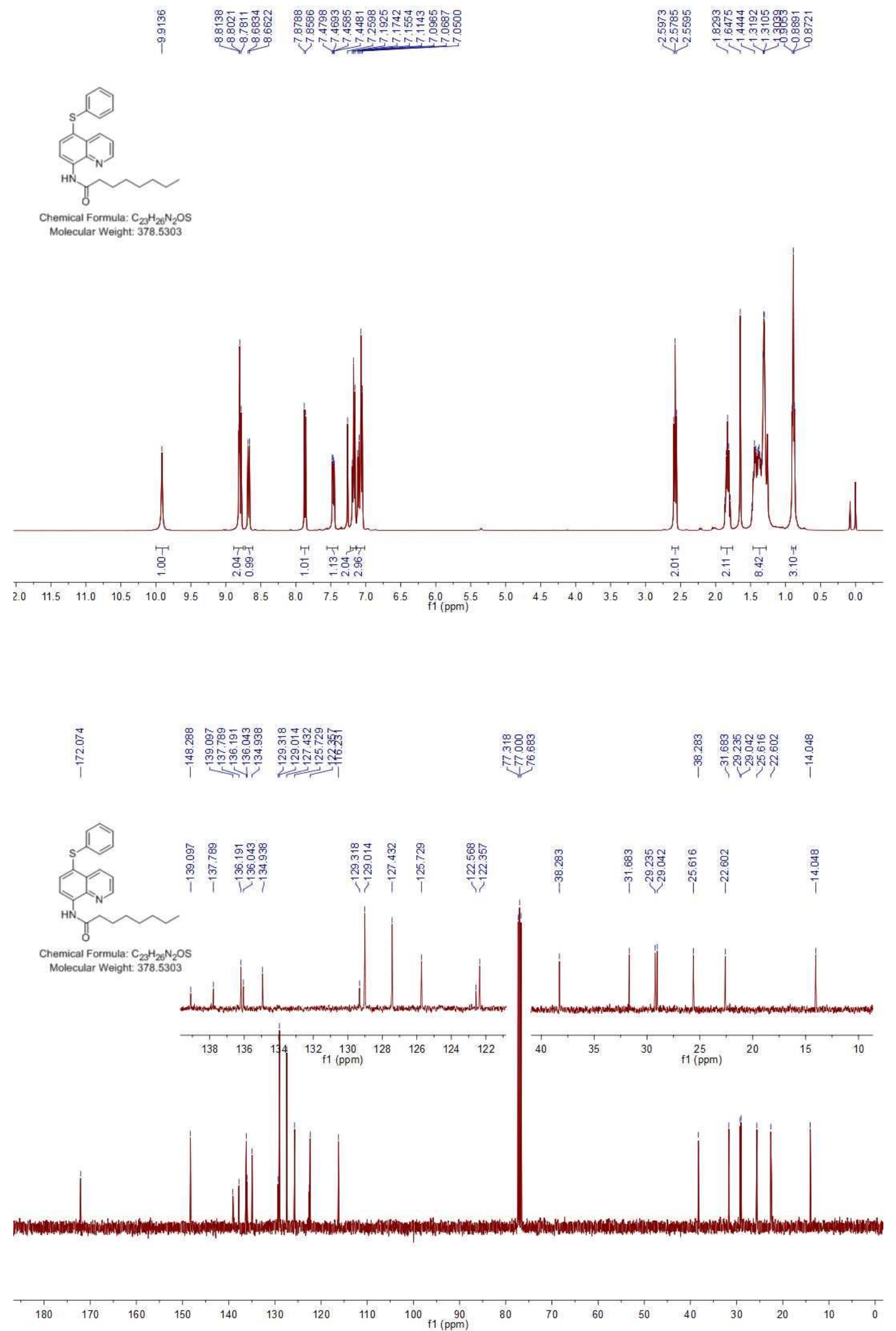
11-(phenylthio)- $N$-(5-(phenylthio)quinolin-8-yl)undecanamide (4p)
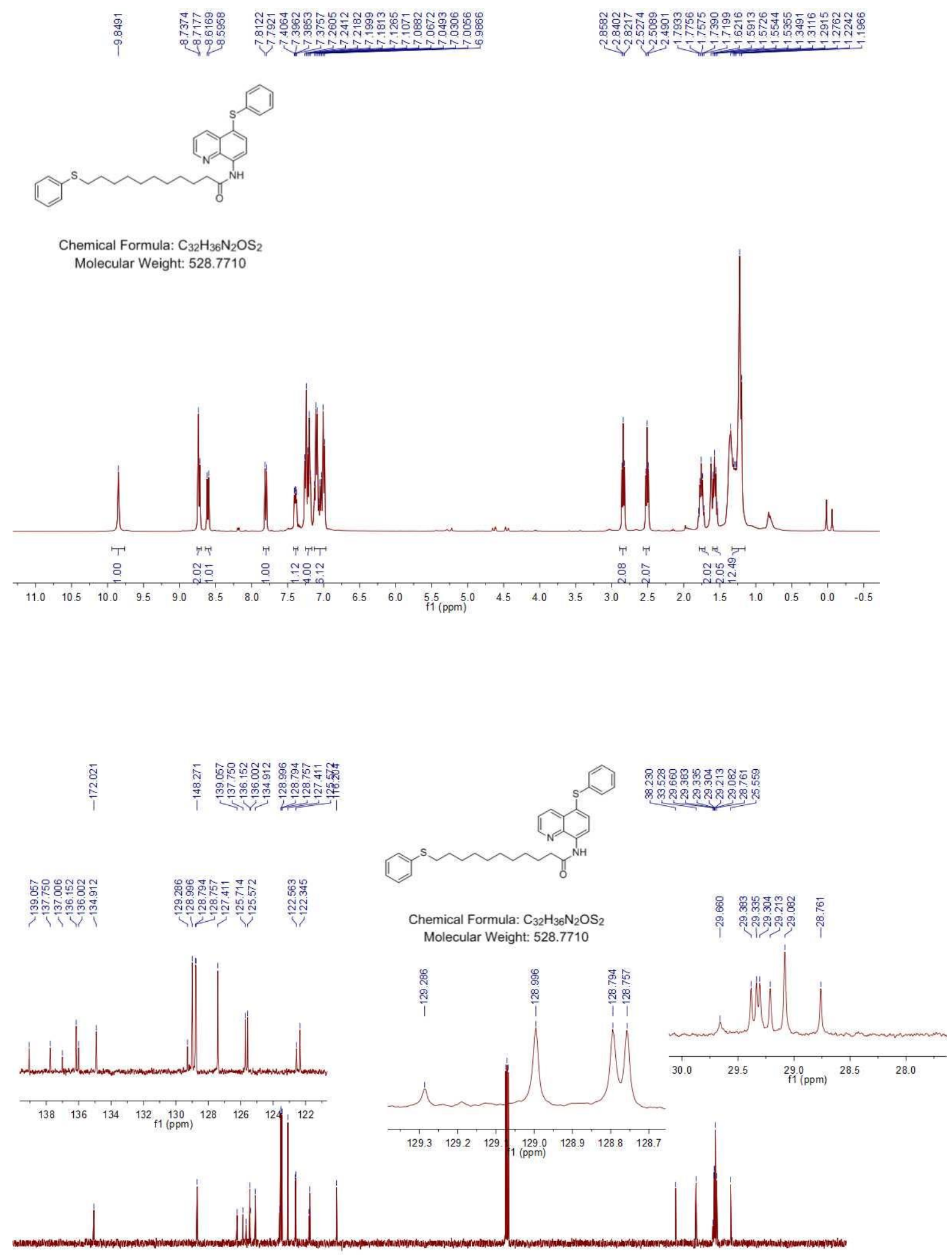

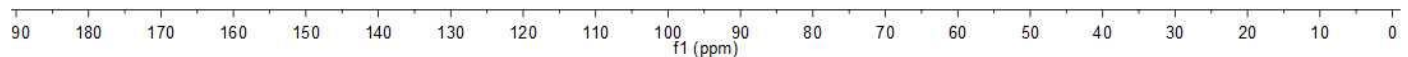


$N$-(5-(phenylselanyl)quinolin-8-yl)pivalamide (5a)

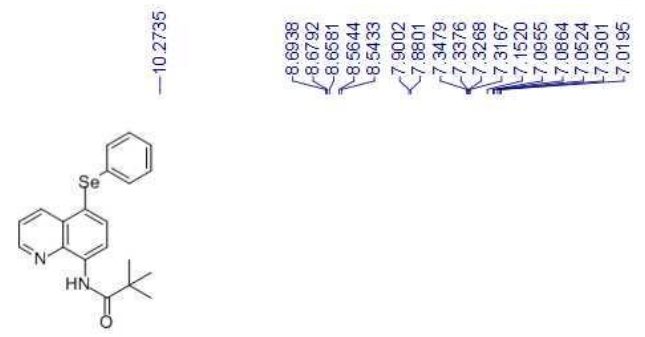

Chemical Formula: $\mathrm{C}_{20} \mathrm{H}_{20} \mathrm{~N}_{2} \mathrm{OSe}$

Molecular Weight: 383.3456

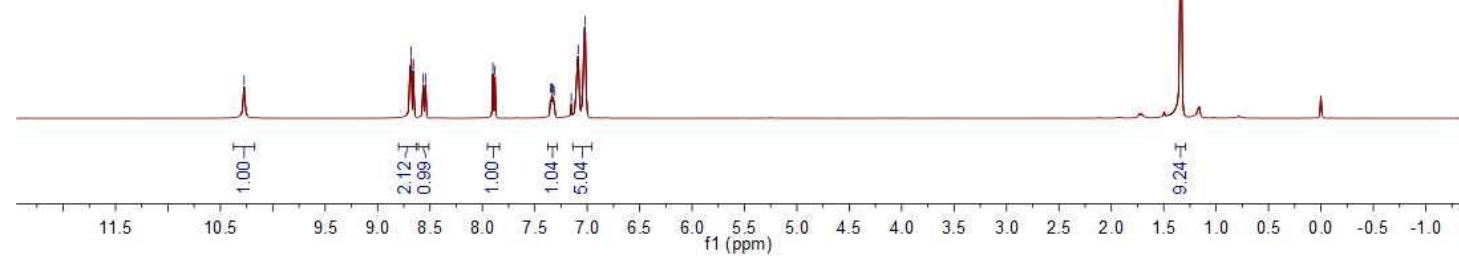

$\begin{array}{ll}0 & 0 \\ 0 & 0\end{array}$

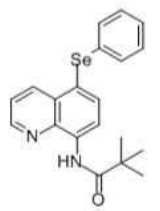

Chemical Formula: $\mathrm{C}_{20} \mathrm{H}_{20} \mathrm{~N}_{2} \mathrm{OS}$ Molecular Weight: 383.3456

잋:

密

200

190

$180 \quad 170 \quad 160$

$\begin{array}{llllll}150 & 140 & 130 & 120 & 110 & 100 \\ \mathrm{f} 1(\mathrm{ppm}) & 1\end{array}$

$90 \quad 80$

60

40

$\begin{array}{llll}30 & 20 & 10 & 0\end{array}$ 
$N$-(5-((4-chlorophenyl)selanyl)quinolin-8-yl)pivalamide (5b)
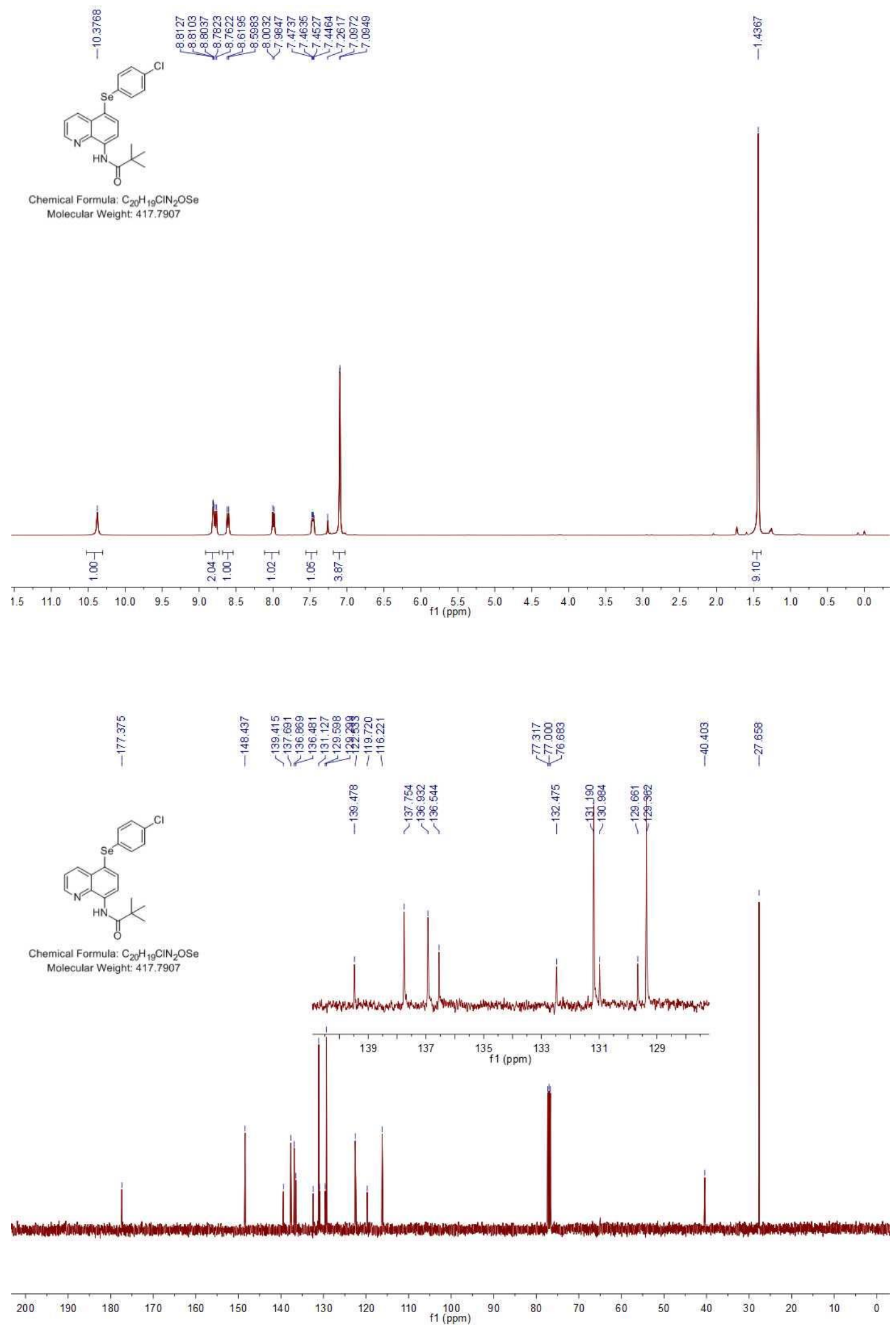
$N-(5-((2,6-$ dimethylphenyl)selanyl)quinolin-8-yl)pivalamide (5c)
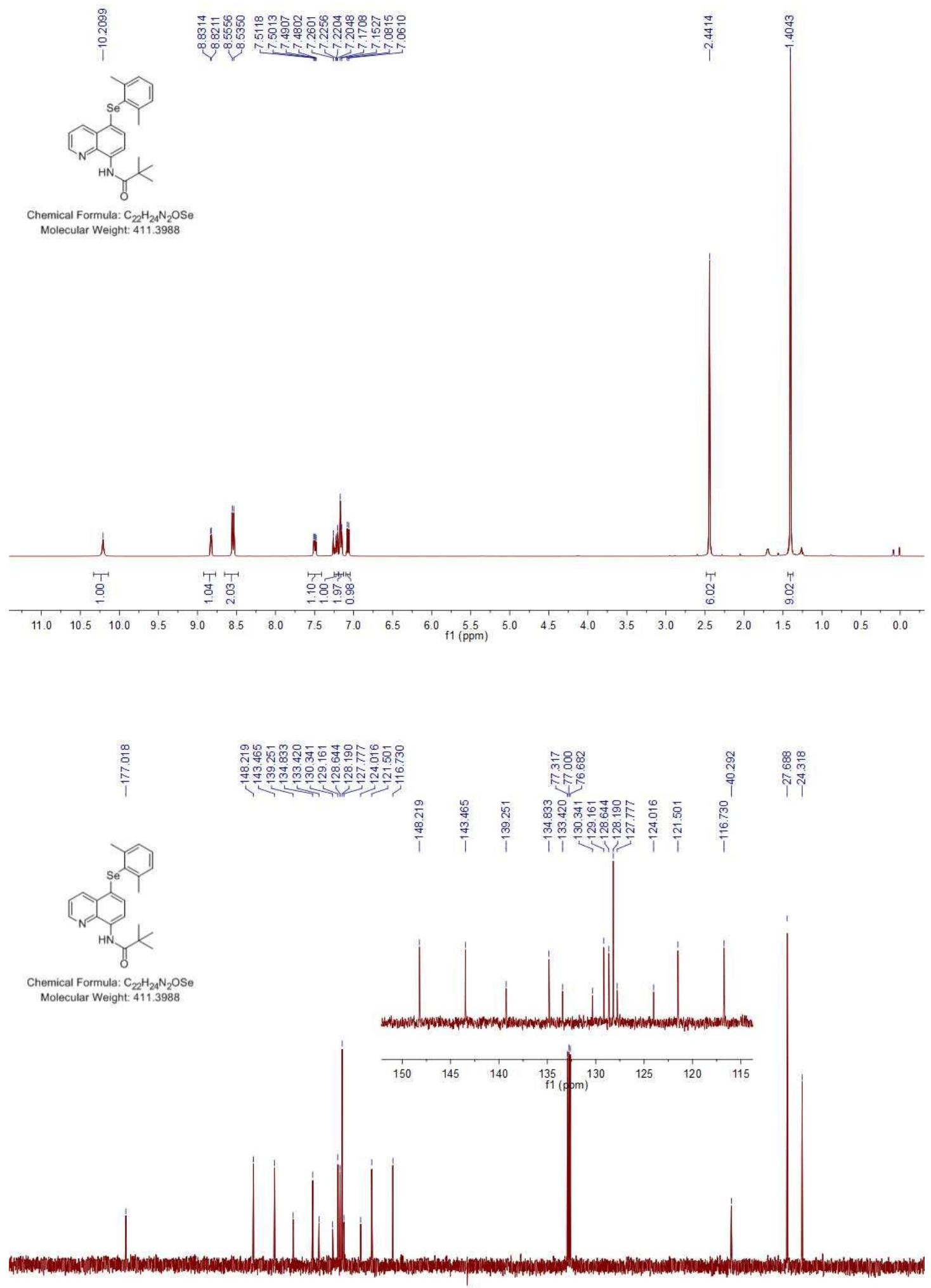

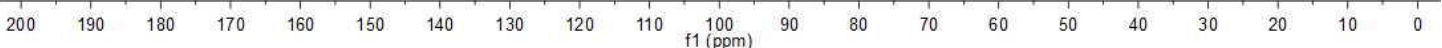


$N$-(5-(mesitylselanyl)quinolin-8-yl)pivalamide(5d)
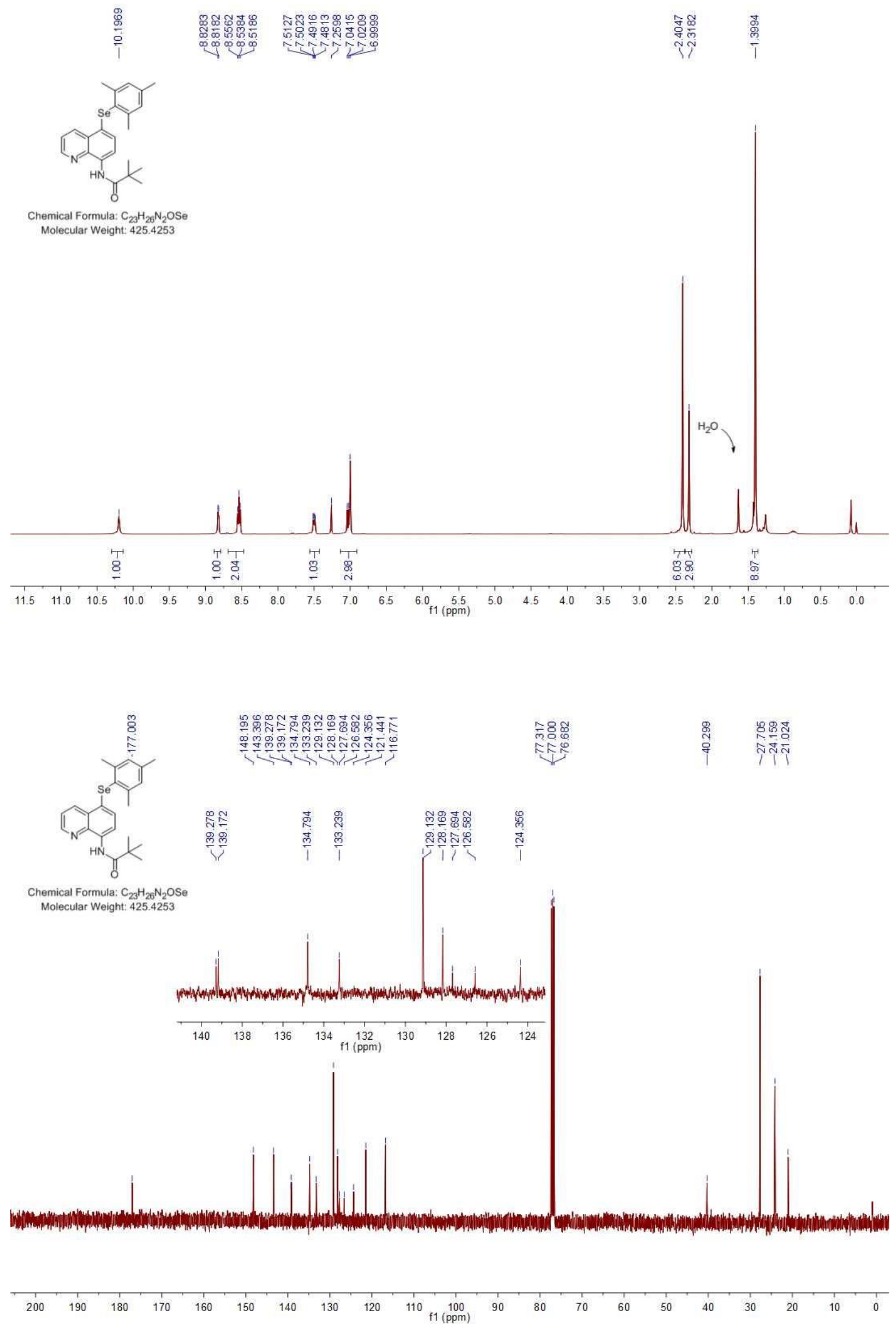
$N-(5-((4-$ chlorophenyl)selanyl)quinolin-8-yl)-2,2-dimethylbutanamide (5e)
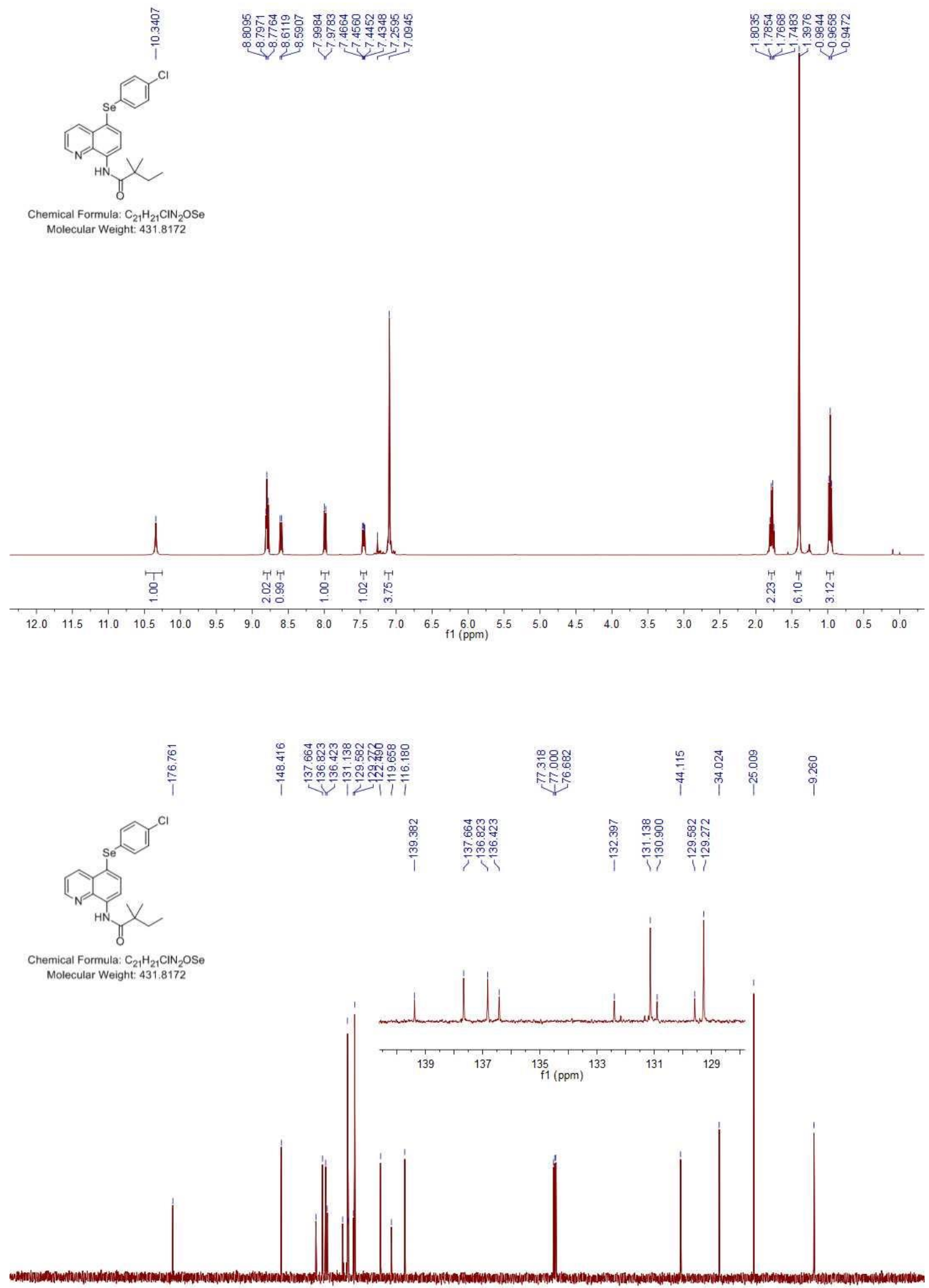

$\begin{array}{lllllllllllllllllllllllllllllllll}210 & 200 & 190 & 180 & 170 & 160 & 150 & 140 & 130 & 120 & 110 & 100 & 90 & 80 & 70 & 60 & 50 & 40 & 30 & 20 & 10 & 0 & -10\end{array}$ 
$N$-(5-((4-chlorophenyl)selanyl)quinolin-8-yl)acetamide (5f)
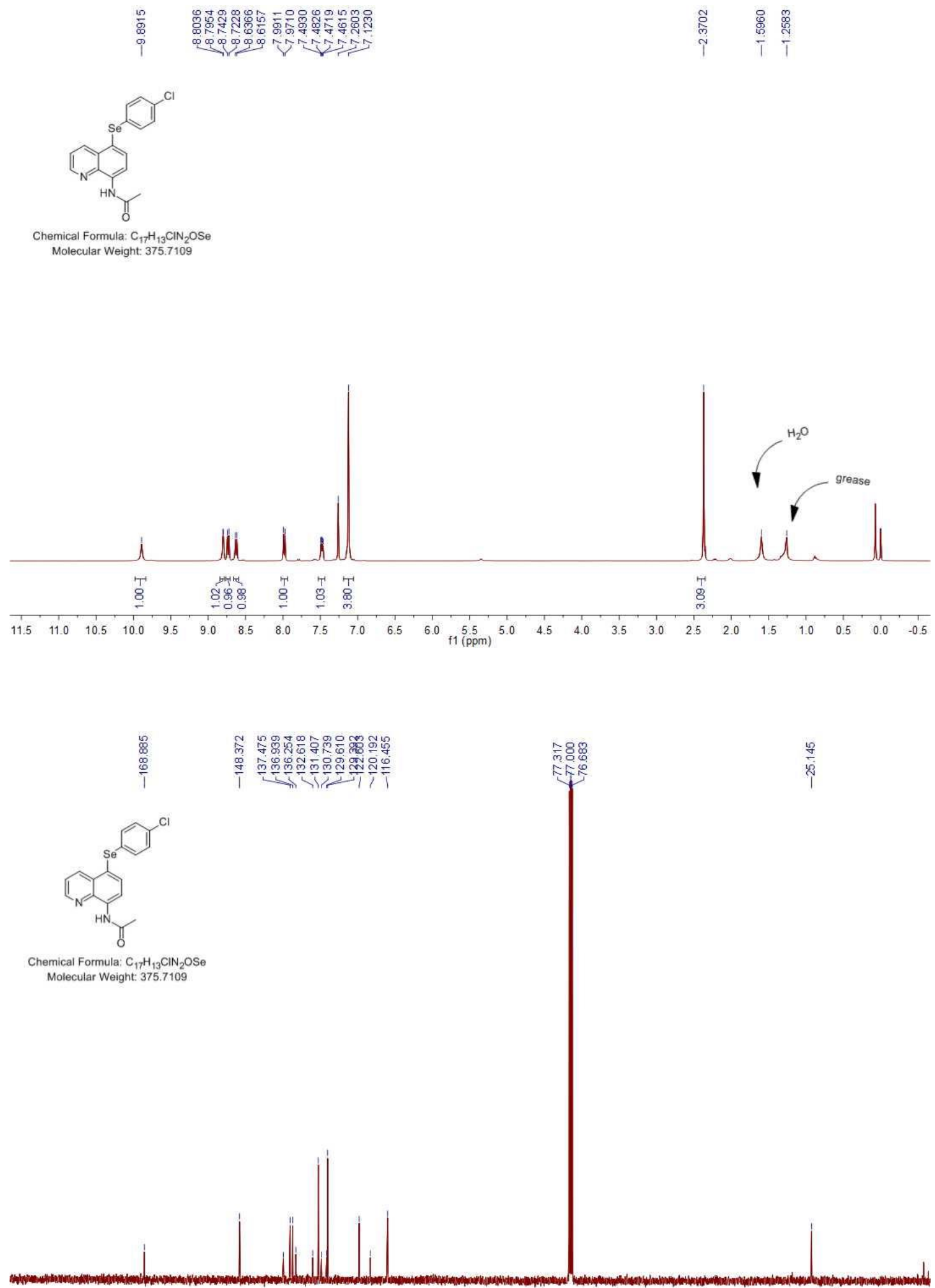

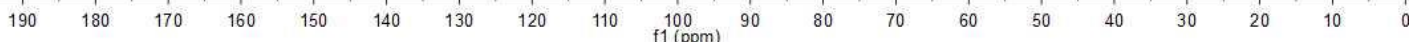


$N$-(5-((4-chlorophenyl)selanyl)quinolin-8-yl)cyclopentanecarboxamide (5g)
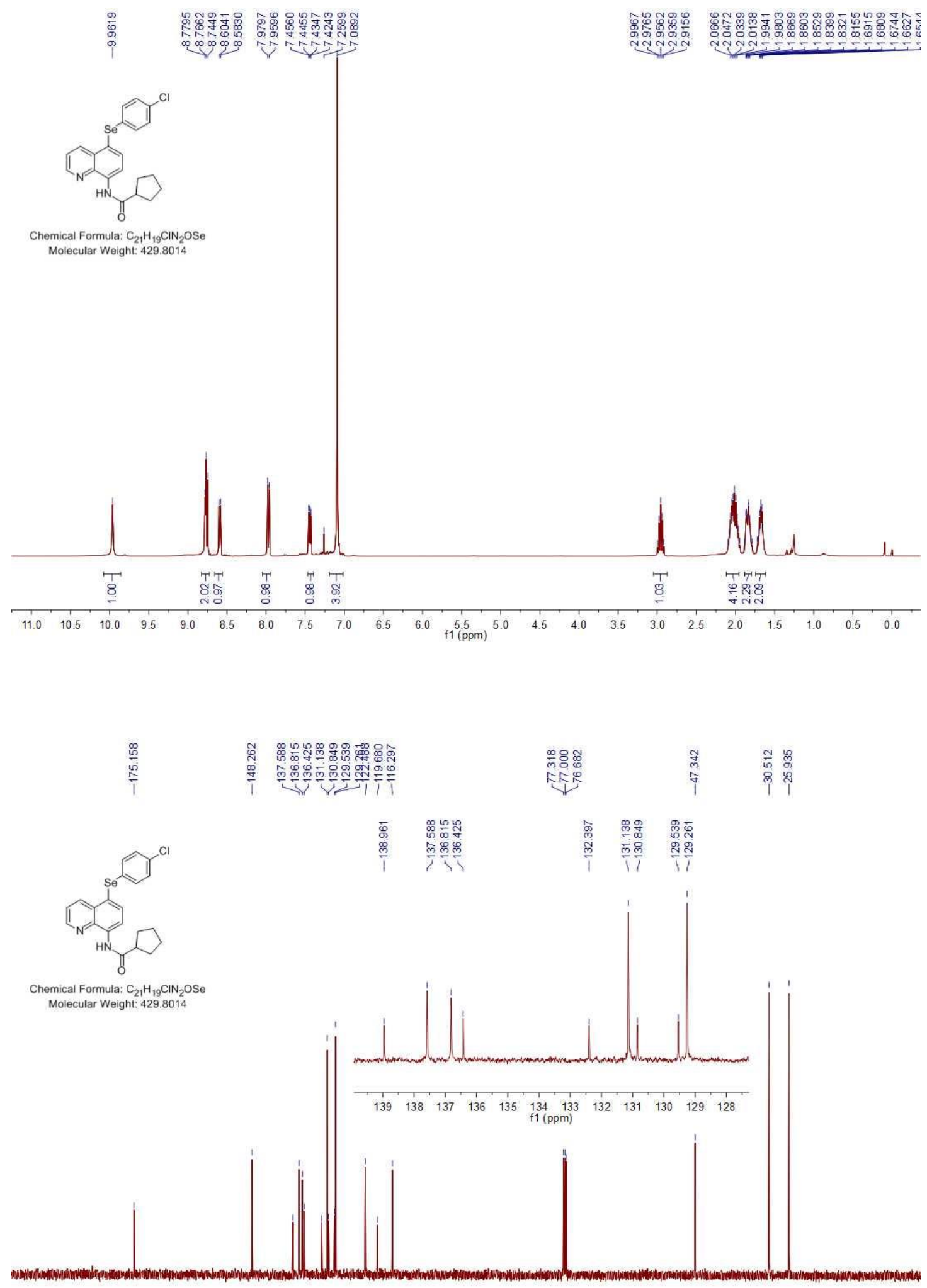

$\begin{array}{lllllllllllllllllllllllll}200 & 190 & 180 & 170 & 160 & 150 & 140 & 130 & 120 & 110 & 100 & 90 & 80 & 70 & 60 & 50 & 40 & 30 & 20 & 10 & 0\end{array}$ 
5-(phenylthio)quinolin-8-amine (6)
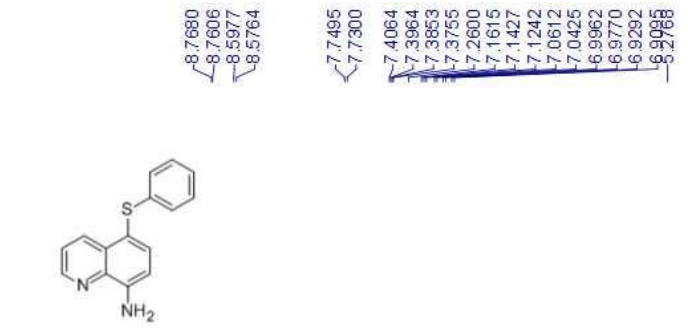

Chemical Formula: $\mathrm{C}_{15} \mathrm{H}_{12} \mathrm{~N}_{2} \mathrm{~S}$

Molecular Weight: 252.3342

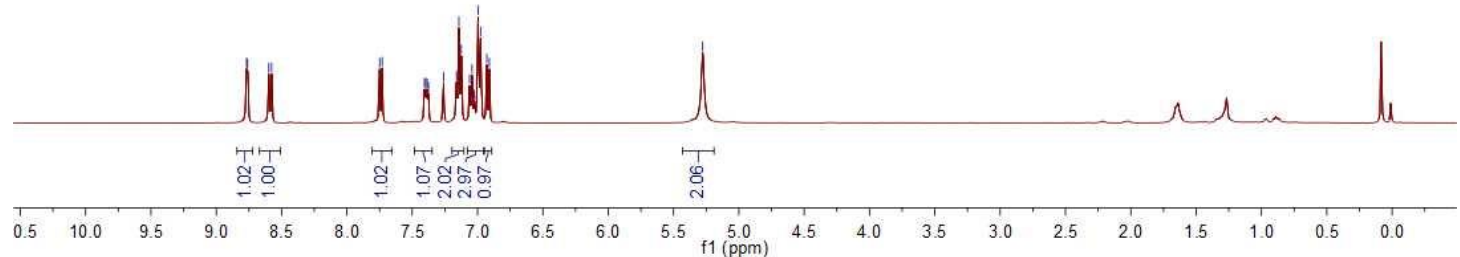

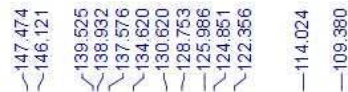

잉요

积唯

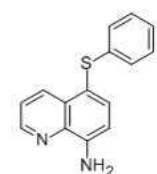

Chemical Formula: $\mathrm{C} 15 \mathrm{H} 12 \mathrm{~N} 2 \mathrm{~S}$

Molecular Weight: 252.3342

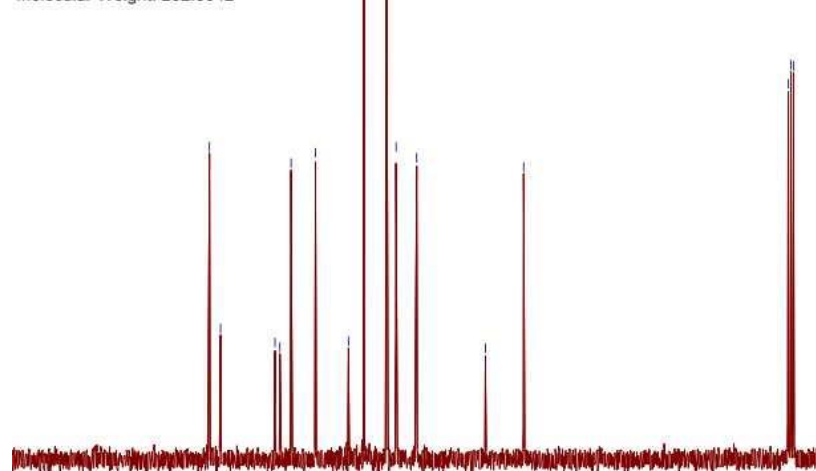

$\begin{array}{lllllllll}170 & 160 & 150 & 140 & 130 & 120 & 110 & 100 & 90\end{array}$

$\begin{array}{llllllll}70 & 60 & 50 & 40 & 30 & 20 & 10 & 0\end{array}$ 
$N$-(5-(phenylsulfonyl)quinolin-8-yl)pivalamide (7)
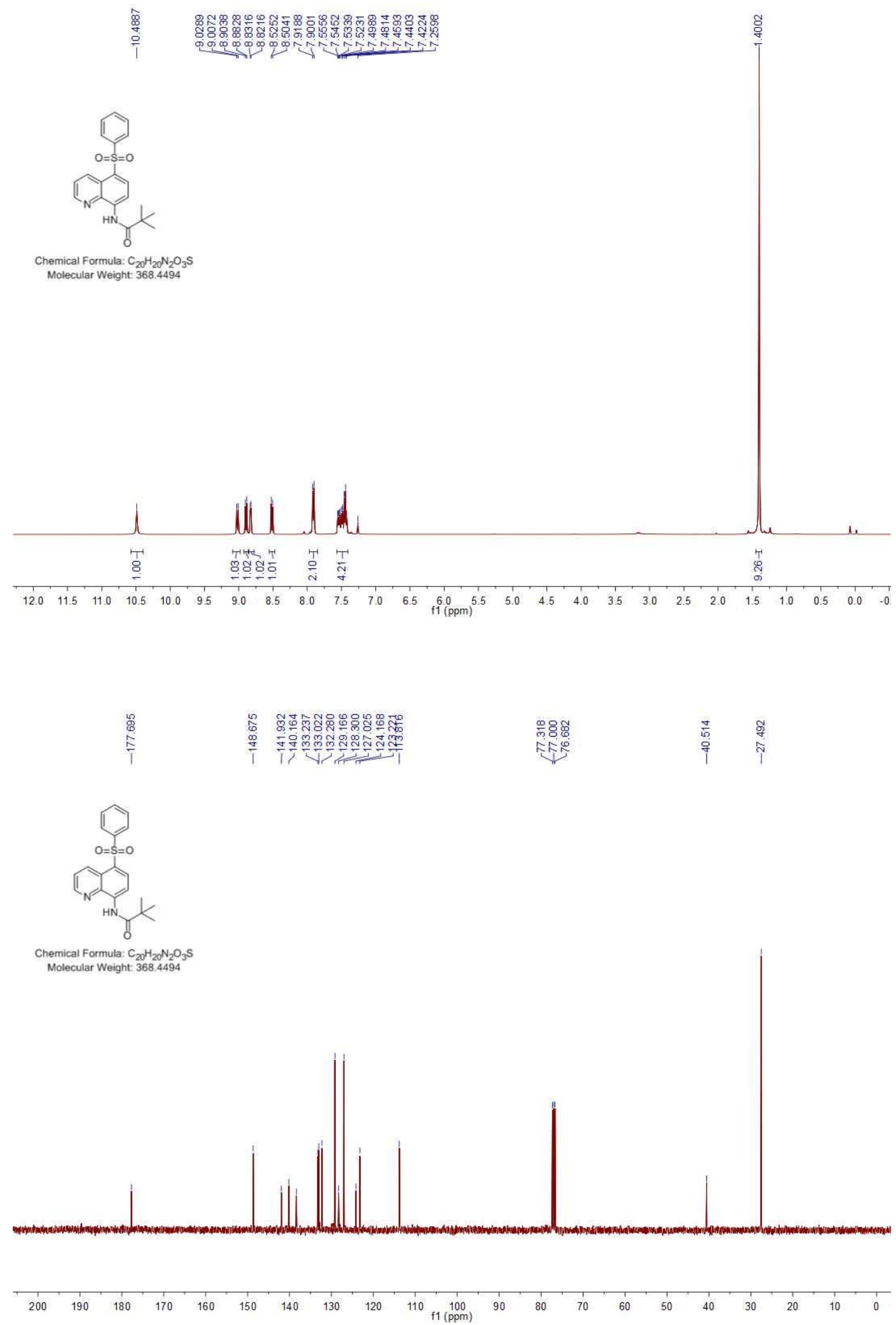
2,2-dimethyl-3-(phenylthio)- $N$-(5-(phenylthio)quinolin-8-yl)propanamide (8)
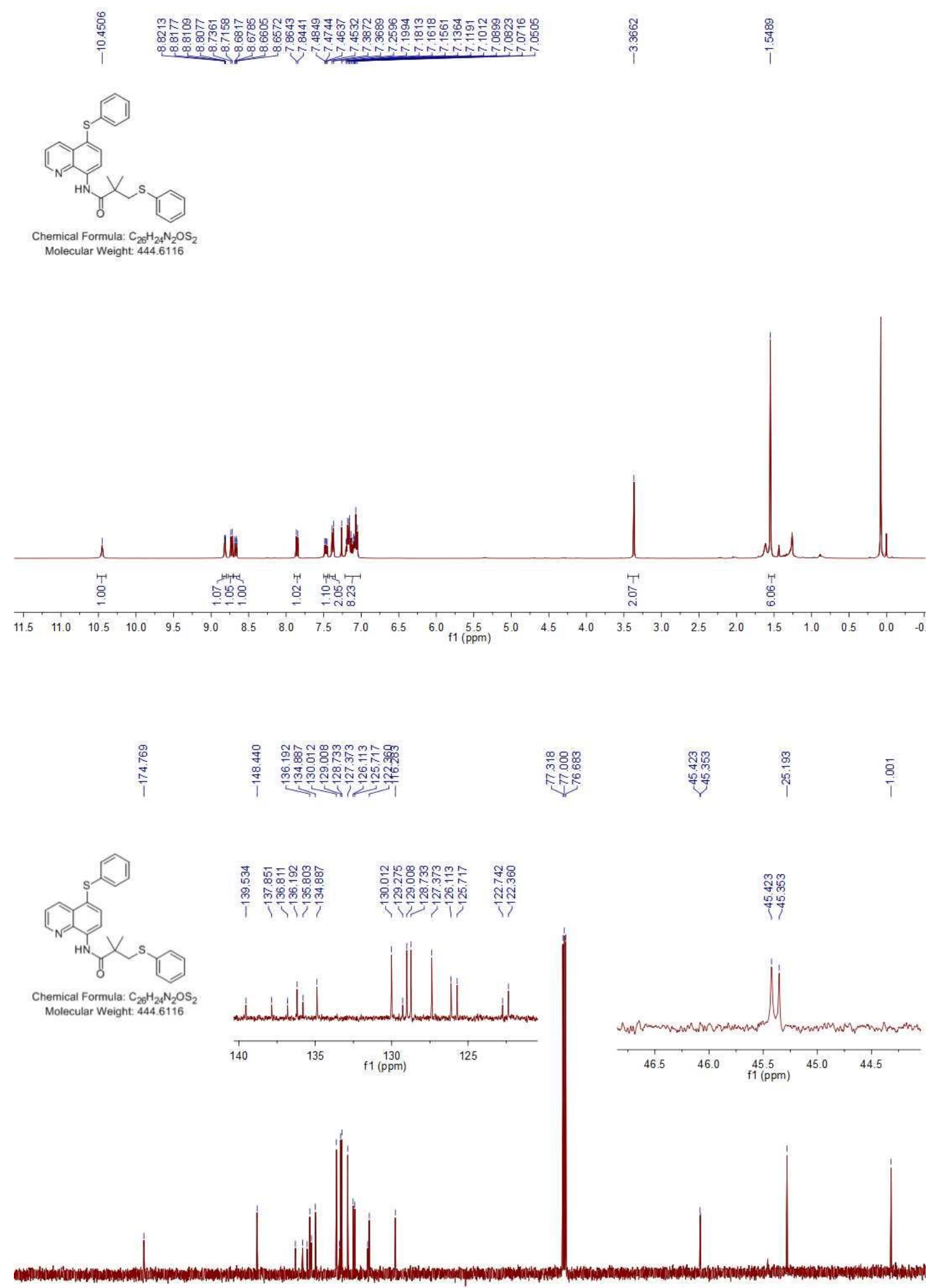

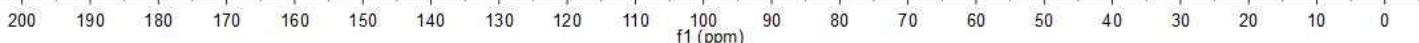

